\title{
An Unbounded Generalization of the Tomita-Takesaki Theory
}

\author{
Atsushi INOUE*
}

\section{\$. Introduction}

Algebras of unbounded operators have been studying by many mathematicians (Borchers, Uhlmann, Lassner, Powers, Schmüdgen, Antoine, Gudder, etc....) from situations of the physical applications as well as the sheer mathematical interest. The study of oneparameter automorphism groups and dynamics in unbounded operator algebras seems to be hardly done except $[8,17]$. It is well known that the Tomita-Takesaki theory plays an important role for such a study in von Neumann algebras. In this direction we consider an unbounded generalization of the Tomita-Takesaki theory, and treat modular automorphism groups of such algebras.

We define the notion of unbounded left Hilbert algebras which is an unbounded generalization of left Hilbert algebras in the sense that the left multiplication is not necessarily bounded. Then a bicommutant $\mathfrak{A}^{\prime \prime}$ of an unbounded left Hilbert algebra $\mathfrak{A}$ is defined and becomes an achieved left Hilbert algebra, and so it induces the fundamental theorem of Tomita for the left von Neumann algebra $\mathscr{U}_{0}\left(\mathfrak{U}^{\prime \prime}\right)$ and the right von Neumann algebra $\mathscr{V}_{0}\left(\mathfrak{Y}^{\prime \prime}\right)$ of $\mathfrak{U}^{\prime \prime}: J^{\prime \prime} \mathscr{U}_{0}\left(\mathfrak{U}^{\prime \prime}\right) J^{\prime \prime}=\mathscr{V}_{0}\left(\mathfrak{U}^{\prime \prime}\right)$, $\Delta^{\prime \prime i t} \mathscr{U}_{0}\left(\mathfrak{U}^{\prime \prime}\right) \Delta^{\prime \prime}-i t=\mathscr{U}_{0}\left(\mathfrak{U}^{\prime \prime}\right)$ for all $t \in \mathbb{R}$, where $J^{\prime \prime}$ is the modular conjugation operator of $\mathfrak{U}^{\prime \prime}$ and $\Delta^{\prime \prime}$ is the modular operator of $\mathfrak{U}^{\prime \prime}$. The first purpose is to extend the above results to an unbounded left Hilbert algebra $\mathfrak{A}$. The following question arises.

Question A. Do there exist $O_{p}^{*}$-algebras $\mathscr{U}(\mathfrak{U})$ and $\mathscr{V}(\mathfrak{U})$ such that $\mathscr{U}(\mathfrak{U})^{\prime \prime}=\mathscr{U}_{0}\left(\mathfrak{T}^{\prime \prime}\right), \mathscr{V}(\mathfrak{V})^{\prime \prime}=\mathscr{V}_{0}\left(\mathfrak{U}^{\prime \prime}\right), J^{\prime \prime} \mathscr{U}(\mathfrak{U}) J^{\prime \prime}=\mathscr{V}(\mathfrak{U})$ and $\Delta^{\prime \prime i t} \mathscr{U}(\mathfrak{U}) d^{\prime \prime}-i t=$

Communicated by H. Araki, December 19, 1985.

*Department of Applied Mathematics Fukuoka University Fukuoka 814-01 Japan 
$=\mathscr{U}(\mathfrak{R})$ for all $t \in \boldsymbol{R}$ ?

In order to solve Question A, we need an unbounded generalization of von Neumann algebras called generalized von Neumann algebra, and further have to consider the invariance of domains under the unitary group $\left\{d^{\prime \prime i t}\right\}$. From this viewpoint, in Section 3 we define the notion of modular unbounded left Hilbert algebras, and show that Question A is affirmative for such an algebra. Thus the notion of modular is important for our study, so that it is natural to consider the following question.

Question B. For each achieved left Hilbert algebra $\mathfrak{A}_{0}$ does there exist a modular unbounded left Hilbert algebra $\mathfrak{A}$ such that $\mathfrak{H}^{\prime \prime}=\mathfrak{Y}_{0}$ ?

In Section 4 we consider Question B for von Neumann algebras $\mathscr{M}_{0}$ with cyclic and separating vector $\xi_{0}$, and show that if the fixedpoint algebra $\mathscr{M}_{0}^{o}$ of the modular automorphism group of the left Hilbert algebra $\mathscr{M}_{0} \xi_{0}$ in $\mathscr{M}_{0}$ is infinitely dimentional, then Question $B$ is affirmative.

Gudder and Hudson have studied positive linear functionals on the canonical algebra $\mathscr{A}$ for one degree of freedom which induce unbounded representations of $\mathscr{A}$ on the Hilbert space of HilbertSchmidt operators [8]. In Section 5 we investigate under what conditions trace functionals on $\mathrm{O}_{p}^{*}$-algebras which are important in states in quantum physics induce modular unbounded left Hilbert algebras using Gudder and Hudson's idea, and apply this result to strongly positive linear functionals on the $\mathrm{O}_{p}^{*}$-algebra $\mathscr{L}^{\dagger}(\mathscr{S}), \mathscr{S}$ being the Schwartz space, and the $\mathrm{O}_{p}^{*}$-algebra generated by the position and the moment operators.

In Section 6 we show that modular unbounded left Hilbert algebras $K^{\prime} \mathfrak{B}$ are constructed by unbounded Hilbert algebras $\mathfrak{B}$ investigated in $[9,14]$ and positive self-adjoint operators $K^{\prime}$ satisfying some conditions and give the necessary and sufficient conditions under which an unbounded left Hilbert algebra $\mathfrak{A}$ is represented as $K^{\prime} \mathfrak{B}$.

\section{§2. Generalized von Neumann Algebras}

We begin with the definitions and the basic properties about $\mathrm{O}_{\mathscr{P}}^{*}$-algebras. Let $\mathscr{D}$ be a dense subspace in a Hilbert space $\mathfrak{S}$. We denote by $\mathscr{L}(\mathscr{D})$ the set of all linear operators defined on $\mathscr{D}$ 
and leave $\mathscr{D}$ invariant, and by $\mathscr{L}^{\dagger}(\mathscr{D})$ the set of all elements $X$ of $\mathscr{L}(\mathscr{D})$ such that the adjoint $X^{*}$ of $X$ exists and the restriction $X^{\dagger}$ of $X^{*}$ to $\mathscr{D}$ is contained in $\mathscr{L}(\mathscr{D})$. Then $\mathscr{L}(\mathscr{D})$ is an algebra under the usual operations, and $\mathscr{L}^{\dagger}(\mathscr{D})$ is a $*$-algebra with involution $X \rightarrow X^{\dagger}$. A $*$-subalgebra of $\mathscr{L}^{\dagger}(\mathscr{D})$ is said to be an $\mathrm{O}_{p}^{*}$-algebra on $\mathscr{D}$.

Let $\mathscr{M}$ be an $\mathrm{O}_{p}^{*}$-algebra on $\mathscr{D}$. A locally convex topology on $\mathscr{D}$ generated by the family of seminorm: $\xi \rightarrow\|\xi\|+\|X \xi\|$ for $X \in \mathscr{M}$, is said to be the induced topology on $\mathscr{D}$, which is denoted by $t_{\mathscr{M}}$. If the locally convex space $\left(\mathscr{D}, t_{\mathscr{M}}\right)$ is complete, then $\mathscr{M}$ is said to be closed. It is well-known that $\mathscr{M}$ is closed if and only if $\mathscr{D}=\cap_{x \in \mathscr{M}} \mathscr{D}(\bar{X})$. If $\mathscr{D}=\underset{x \in \mathscr{M}}{\cap \mathscr{D}}\left(X^{*}\right)$, then $\mathscr{M}$ is said to be self-adjoint. If $X^{*}=\bar{X}^{\dagger}$ for each $X \in \mathscr{M}$, then $\mathscr{M}$ is said to be standard. It is clear that if $\mathscr{M}$ is standard then it is self-adjoint.

In order to generalize the notion of von Neumann algebras to the unbounded case, we give some topologies on $\mathscr{L}^{\dagger}(\mathscr{D})$ and commutants of $\mathrm{O}_{p}^{*}$-algebras. A locally convex topology on $\mathscr{L}^{\dagger}(\mathscr{D})$ generated by the family of seminorms: $P_{\xi, \eta}(X)=|(X \xi \mid \eta)|$ for $\xi, \eta \in \mathscr{D}$ (resp. $P_{\xi}(X)=i|X \xi i|$ for $\xi \in \mathscr{D}, P_{\xi}^{*}(X)=\|X \xi\|+\left\|X^{\dagger} \xi\right\|$ for $\left.\xi \in \mathscr{D}\right)$ is said to be the weak topology (resp. the strong topology, the strong*-topology), which is denoted by $t_{w}\left(\operatorname{resp} . t_{s}, t_{s}^{*}\right)$. We now introduce stronger topologies than these topologies. We put

$$
P_{Y, \xi}(X)=\|Y X \xi\|_{1}, P_{Y, \xi}^{*}(X)=\|Y X \xi\|+\left\|Y X^{\dagger} \xi\right\|
$$

for $X, Y \in \mathscr{L}^{\dagger}(\mathscr{D})$ and $\xi \in \mathscr{D}$. A locally convex topology on $\mathscr{L}^{\dagger}(\mathscr{D})$ generated by the family $\left\{P_{y, \xi}(\bullet) ; Y \in \mathscr{L}^{\dagger}(\mathscr{D}), \xi \in \mathscr{D}\right\}$ (resp. $\left\{P_{Y, \xi}^{*}(\bullet)\right.$; $\left.Y \in \mathscr{L}^{\dagger}(\mathscr{D}), \xi \in \mathscr{D}\right\}$ ) is said to be the ultra strong topology (resp. the ultra strong*-topology), which is denoted by $t_{u s}$ (resp. $t_{u s}^{*}$ ). It is easy to prove the following

Lemma 2.1. Suppose $\mathscr{D}$ is a dense subspace in a Hilbert space $\mathfrak{H}$ such that $\mathscr{L}^{\dagger}(\mathscr{D})$ is closed. Then $\left(\mathscr{L}^{\dagger}(\mathscr{D}), t_{u s}^{*}\right)$ is a complete locally convex *-algebra. The closure $\overline{\mathscr{M}}^{*}$ us of an $O_{p}^{*}$-algebra $\mathscr{M}$ on $\mathscr{D}$ with respect to the topology $t_{u s}^{*}$ is an $O_{p}^{*}$-algebra on $\mathscr{D}$.

Definition 2.2. An $O_{p}^{*}$-algebra $\mathscr{M}$ on $\mathscr{D}$ is said to be a generalized $W^{*}$-algebra if $\mathscr{M}=\overline{\mathscr{M}}^{t^{*}}$. 
Suppose $\mathscr{L}^{\dagger}(\mathscr{D})$ is closed. By Lemma $2.1{\mathscr{M}^{t}}^{*}$ is a generalized $\mathrm{W}^{*}$-algebra on $\mathscr{D}$ for every $\mathrm{O}_{p}^{*}$-algebra $\mathscr{M}$ on $\mathscr{D}$.

We define a commutant $\mathscr{M}_{w}^{\prime}$ (simply, $\mathscr{M}^{\prime}$ ) of an $\mathrm{O}_{p}^{*}$-algebra $\mathscr{M}$ on $\mathscr{D}$ as follows:

$$
\begin{aligned}
\mathscr{M}^{\prime}=\{C \in \mathscr{B}(\mathscr{S}) ; & (C X \xi \mid \eta)=\left(C \xi \mid X^{\dagger} \eta\right) \\
& \text { for each } \xi, \eta \in \mathscr{D} \text { and } X \in \mathscr{M}\},
\end{aligned}
$$

where $\mathscr{B}(\mathscr{S})$ is the set of all bounded operators on $\mathscr{S}$. It is wellknown that $\mathscr{M}^{\prime}$ is a weakly closed, *-invariant subspace of $\mathscr{B}(\mathscr{S})$, but it is not necessarily an algebra, and for the following statements:

(1) $\mathscr{M}$ is self-adjoint;

(2) $\mathscr{M}^{\prime} \mathscr{D}=\mathscr{D}$;

(2) ' $\bar{X}$ is affiliated with $\mathscr{M}^{\prime \prime}$ for each $X \in \mathscr{M}$;

(3) $\mathscr{M}^{\prime}$ is a von Neumann algebra,

the implications<smiles>[As]=[W]</smiles>

hold, but the converse implications don't necessarily hold [7, 15, 23]. Further, for the above statements (2) and (3) we have the following

Lemma 2. 3. [16] Suppose $\mathscr{M}$ is an $O_{p}^{*}$-algebra on $\mathscr{D}$ such that $\mathscr{M}^{\prime}$ is a von Neumann algebra. Put

$$
\begin{aligned}
& \mathscr{D}_{1}=\left\{\sum_{i=1}^{n} C_{i} \xi_{i} ; C_{i} \in \mathscr{M}^{\prime}, \xi_{i} \in \mathscr{D}\right\}, \\
& X_{1}\left(\sum_{i=1}^{n} C_{i} \xi_{i}\right)=\sum_{i=1}^{n} C_{i} X \xi_{i}, X \in \mathscr{M},\left\{C_{i}\right\} \subset \mathscr{M},\left\{\xi_{i}\right\} \subset \mathscr{D} ; \\
& \mathscr{M}_{1}=\left\{X_{1} ; X \in \mathscr{M}\right\} .
\end{aligned}
$$

Then the closure $(\hat{\mathscr{M}}, \hat{\mathscr{D}})$ of the $O_{p}^{*}$-algebra $\left(\mathscr{M}_{1}, \mathscr{D}_{1}\right)$ is the minimum closed extension of $(\mathscr{M}, \mathscr{D})$ such that $\hat{\mathscr{M}}^{\prime}=\mathscr{M}^{\prime}$ and $\mathscr{M}^{\prime} \hat{\mathscr{D}}=\hat{\mathscr{D}}$.

We next define unbounded commutants $\mathscr{M}_{\sigma}^{\prime}, \mathscr{M}_{c}^{\prime}$ and unbounded bicommutants $\mathscr{M}_{w c,}^{\prime \prime} \mathscr{M}_{c c}^{\prime \prime}$ of an $\mathrm{O}_{p}^{*}$-algebra $\mathscr{M}$ on $\mathscr{D}$ as follows:

$$
\begin{gathered}
\mathscr{M}_{\sigma}^{\prime}=\left\{S \in \mathscr{C}^{\dagger}(\mathscr{D}, \mathscr{S}) ;(S X \xi \mid \eta)=\left(S \xi \mid X^{\dagger} \eta\right),\right. \\
\text { for each } \xi, \eta \in \mathscr{D} \text { and } X \in \mathscr{M}\}, \\
\mathscr{M}_{c}^{\prime}=\left\{S \in \mathscr{L}^{\dagger}(\mathscr{D}) ; S X=X S \text { for each } X \in \mathscr{M}\right\},
\end{gathered}
$$




$$
\begin{aligned}
& \mathscr{M}_{w c}^{\prime \prime}=\left\{X \in \mathscr{L}^{\dagger}(\mathscr{D}) ;\right.(C X \xi \mid \eta)=\left(C \xi \mid X^{\dagger} \eta\right) \\
&\text { for each } \left.\xi, \eta \in \mathscr{D} \text { and } C \in \mathscr{M}^{\prime}\right\}, \\
& \mathscr{M}_{c c}^{\prime \prime}=\left\{X \in \mathscr{L}^{\dagger}(\mathscr{D}) ; X S=S X \text { for each } S \in \mathscr{M}_{c}^{\prime}\right\},
\end{aligned}
$$

where $\mathscr{C}^{\uparrow}(\mathscr{D}, \mathfrak{S})$ is the set of all linear operators $X$ in $\mathscr{S}$ such that $\mathscr{D}(X) \cap \mathscr{D}\left(X^{*}\right) \supset \mathscr{D}$. The study of unbounded commutants has been treated in $[5,7,8,10,16,21]$, in particular Mathot has investigated topological properties of unbounded commutants of $\mathrm{O}_{p}^{*}$-algebras [21]. We have the following

Lemma 2.4. $[16,21]$ (1) $\mathscr{M}_{\sigma}^{\prime}$ is a subspace of $\mathscr{C}^{\dagger}(\mathscr{D}, \mathfrak{S})$ whose bounded part equals $\mathscr{M}^{\prime}$.

(2) $\mathscr{M}_{c}^{\prime}$ is an $O_{p}^{*}$-algebra on $\mathscr{D}$ contained in $\mathscr{M}_{\sigma}^{\prime}$.

(3) $\mathscr{M}_{w c}^{\prime \prime}$ is an $O_{p}^{*}$-algebra on $\mathscr{D}$ containing the closure $\overline{\mathscr{M}}^{t^{*}}$ of $\mathscr{M}$ in $\left(\mathscr{L}^{\dagger}(\mathscr{D}), t_{s}^{*}\right)$ such that $\left(\mathscr{H}_{w c}^{\prime \prime}\right)^{\prime}=\mathscr{M}^{\prime}$.

(4) $\mathscr{M}_{c c}^{\prime \prime}$ is an $O_{p}^{*}$-algebra on $\mathscr{D}$ containing the closure $\bar{M}^{t}$ of $\mathscr{M}$ in $\left(\mathscr{L}^{\dagger}(\mathscr{D}), t_{w}\right)$.

(5) Suppose $\mathscr{M}^{\prime} \mathscr{D}=\mathscr{D}$. Then $\mathscr{M}_{\omega c}^{\prime \prime}=\left\{X \in \mathscr{L}^{\dagger}(\mathscr{D}) ; \bar{X}\right.$ is affiliated with $\left.\mathscr{M}^{\prime \prime}\right\} \supset \mathscr{M}_{c c}^{\prime \prime} \supset \overline{\mathscr{M}}^{t}$.

(6) Suppose $\mathscr{M}$ is an $O_{p}^{*}$-algebra on $\mathscr{D}$ consisting of bounded operators. Then $\mathscr{M}_{w c}^{\prime \prime}={\mathscr{M}^{*}}^{*}$.

Definition 2.5. An $O_{p}^{*}$-algebra $\mathscr{M}$ on $\mathscr{D}$ is said to be a generalized von Neumann algebra if $\mathscr{M}$ is closed, $\mathscr{M}^{\prime} \mathscr{D}=\mathscr{D}$ and $\mathscr{M}_{w c}^{\prime \prime}=\mathscr{M}$.

Remark. (1) Suppose $\mathscr{M}$ is an $O_{p}^{*}$-algebra on $\mathscr{D}$ such that $\mathscr{M}^{\prime}$ is a von Neumann algebra. Then it follows from Lemma 2.3 that $\hat{\mathscr{M}}_{w c}^{\prime \prime}$ is a generalized von Neumann algebra on $\hat{\mathscr{D}}$.

(2) Suppose $\mathscr{M}$ is a generalized von Neumann algebra on $\mathscr{D}$. Then it follows from Lemma 2.4, (5) that $\mathscr{M}=\left\{X \in \mathscr{L}^{\dagger}(\mathscr{D}) ; \bar{X}\right.$ is affiliated with $\left.\mathscr{M}^{\prime \prime}\right\}=\mathscr{M}_{c c}^{\prime \prime}=\overline{\mathscr{M}}^{t}{ }^{t}=\bar{M}^{t^{*}}{ }^{*}$, so that $\mathscr{M}$ is a generalized $W^{*}$-algebra on $\mathscr{D}$. In the bounded case, these notions are equivalent to that of von Neumann algebras.

We next consider relations of the bounded part $\mathscr{M}_{b}$ and $\mathscr{M}^{\prime \prime}$ of an $\mathrm{O}_{p}^{*}$-algebra $\mathscr{M}$, and define the notion of $\mathrm{EW}^{*}$-algebras which is an another unbounded generalization of von Neumann algebras. Let 
$\mathscr{M}$ be an $\mathrm{O}_{p}^{*}$-algebra on $\mathscr{D}$. Put

$$
\begin{aligned}
& \mathscr{M}_{b}=\{A \in \mathscr{M} ; \bar{A} \in \mathscr{B}(\mathscr{S})\}, \\
& \mathscr{M}^{\prime \prime \mathscr{D}}=\left\{A / \mathscr{D} ; A \in \mathscr{M}^{\prime \prime}, A \mathscr{D} \subset \mathscr{D} \text { and } A^{*} \mathscr{D} \subset \mathscr{D}\right\} .
\end{aligned}
$$

Then $\mathscr{M}_{b} \subset \mathscr{M}^{\prime \prime \mathscr{D}}$. Suppose $\mathscr{M}$ is a generalized von Neumann algebra. Then $\mathscr{M}_{b}=\mathscr{M}^{\prime \prime} \mathscr{D}$, but $\overline{\mathscr{M}}_{b}$ and $\mathscr{M}^{\prime \prime}$ have not generally relations any more. We can characterize generalized von Neumann algebras $\mathscr{M}$ on $\mathscr{D}$ satisfying $\mathscr{M}_{b}^{\prime \prime}=\mathscr{M}^{\prime \prime}$ as follows.

Proposition 2.6. Suppose $\mathscr{M}$ is a closed $O_{p}^{*}$-algebra on $\mathscr{D}$ such that $\mathscr{M}^{\prime} \mathscr{D}=\mathscr{D}$. Then $\mathscr{M}$ is a generalized von Neumann algebra on $\mathscr{D}$ satisfying $\mathscr{M}_{b}^{\prime \prime}=\mathscr{M}^{\prime \prime}$ if and only if $\mathscr{M}={\overline{\mathscr{M}^{\prime \prime}}}^{t_{s}^{*}}$.

Proof. Suppose $\mathscr{M}$ is a generalized von Neumann algebra such that $\mathscr{M}_{b}^{\prime \prime}=\mathscr{M}^{\prime \prime}$. Then it follows from Lemma 2.4, (6) that

$$
{\overline{\mathscr{M}^{\prime \prime}}}^{t^{*}}=\left(\mathscr{M}^{\prime \prime \mathscr{D}}\right)_{w c}^{\prime \prime}=\mathscr{M}_{w c}^{\prime \prime}=\mathscr{M} \text {. }
$$

Conversely suppose $\mathscr{M}=\overline{\mathscr{M}}^{\mathscr{D}^{t}}{ }^{*}$. Then $\mathscr{M}^{\prime}=\left(\mathscr{M}^{\prime \prime \mathscr{D}}\right)^{\prime}$. It hence follows from Lemma 2.4, (6) that

$$
\mathscr{M}=\overline{\mathscr{M}}^{t^{t}}{ }^{*}=\left(\mathscr{M}^{\prime \prime \mathscr{D}}\right){ }_{w c}^{\prime \prime}=\mathscr{M}_{w c}^{\prime \prime}
$$

so that $\mathscr{M}$ is a generalized von Neumann algebra such that $\mathscr{M}^{\prime \prime}=\mathscr{M}_{b}^{\prime \prime}$.

We introduce the notion of $\mathrm{EW}^{*}$-algebras which is another unbounded generalization of von Neumann algebras investigated by $[9,12$, 14]. An $\mathrm{O}_{p}^{*}$-algebra $\mathscr{M}$ on $\mathscr{D}$ is said to be an $\mathrm{EW}^{*}$-algebra on $\mathscr{D}$ (over $\overline{\mathscr{M}_{b}}$ ) if $\overline{\mathscr{M}}_{b}$ is a von Neumann algebra on $\mathscr{S}$ and $\left(I+X^{*} \bar{X}\right)^{-1}$ $\in \overline{\mathscr{M}}_{b}$ for each $X \in \mathscr{M}$. It is well-known that a closed EW*-algebra is standard. Let $\mathscr{M}$ be a closed $\mathrm{EW}^{*}$-algebra on $\mathscr{D}$. Then $\mathscr{M}^{t^{*}}$ and $\mathscr{M}_{w c}^{\prime \prime}$ are closed $\mathrm{EW}^{*}$-algebras on $\mathscr{D}$ over $\mathscr{M}^{\prime \prime}$. In particular, $\mathscr{M}_{w c}^{\prime \prime}$ is maximum among $\mathrm{EW}^{*}$-algebras on $\mathscr{D}$ over $\mathscr{M}^{\prime \prime}$, which equals $\{X \in$ $\mathscr{L}^{\dagger}(\mathscr{D}) ; \bar{X}$ is affiliated with $\left.\mathscr{M}^{\prime \prime}\right\}=\mathscr{M}_{c c}^{\prime \prime}=\mathscr{M}^{*^{*}}$.

Let $\mathscr{A}$ be a $*$-algebra. A $*$-homomorphism $\pi$ of $\mathscr{A}$ into $\mathscr{L}^{\dagger}(\mathscr{D}(\pi))$, where $\mathscr{D}(\pi)$ is a dense subspace in a Hilbert space $\mathscr{S}_{\pi}$, is said to be a $*$-representation of $\mathscr{A}$ on $\mathscr{S}_{\pi}$. A $*$-representation $\pi$ of $\mathscr{A}$ is said to be closed (resp. self-adjoint, standard) if the $\mathrm{O}_{p}^{*}$-algebra $\pi(\mathscr{A})$ on $\mathscr{D}(\pi)$ is closed (resp. self-adjoint, standard). Let $\pi$ be a $*$-representation of $\mathscr{A}$. We put 


$$
\begin{aligned}
& \left\{\begin{array}{l}
\mathscr{D}(\tilde{\pi})=\cap \mathscr{D}(\overline{\pi(x)}), \\
\tilde{\pi}(x) \xi=\overline{\pi(x)} \xi, \quad x \in \mathscr{A}, \quad \xi \in \mathscr{D}(\tilde{\pi}) ;
\end{array}\right. \\
& \left\{\begin{array}{l}
\mathscr{D}\left(\pi^{*}\right)=\cap \mathscr{D}\left(\pi(x)^{*}\right), \\
\pi^{*}(x) \xi=\pi\left(x^{*}\right) * \xi, \quad x \in \mathscr{A}, \quad \xi \in \mathscr{D}\left(\pi^{*}\right) ;
\end{array}\right. \\
& \left\{\begin{array}{l}
\mathscr{D}\left(\pi^{* *}\right)=\cap \mathscr{D}\left(\pi^{*}(x)^{*}\right), \\
\pi^{* *}(x) \xi=\pi^{*}\left(x^{*}\right) * \xi, \quad x \in \mathscr{A}, \quad \xi \in \mathscr{D}\left(\pi^{* *}\right) .
\end{array}\right.
\end{aligned}
$$

Then $\tilde{\pi}$ and $\pi^{* *}$ are closed $*$-representations of $\mathscr{A}$ in $\mathfrak{S}_{\pi}$ such that $\tilde{\pi}(\mathscr{A})^{\prime}{ }_{w}=\pi^{* *}(\mathscr{A})_{w}^{\prime}$, and $\pi^{*}$ is a closed representation of $\mathscr{A}$ in $\mathfrak{S}_{\pi}$, but it is not necessarily a $*$-representation. These representations have the relations: $\pi \subset \tilde{\pi} \subset \pi^{* *} \subset \pi^{*}[7,15,23]$.

Let $\phi$ be a positive linear functional on a $*$-algebra $\mathscr{A}$. It is easily shown that $N_{\phi}=\left\{x \in \mathscr{A} ; \phi\left(x^{*} x\right)=0\right\}$ is a left ideal in $\mathscr{A}$. For each $x \in \mathscr{A}$ we cienote by $\lambda_{\phi}(x)$ the coset of $\mathscr{A} / N_{\phi}$ which contains $x$, and define an inner product $(\mid)$ on $\lambda_{\phi}(\mathscr{A})$ by: $\left(\lambda_{\phi}(x) \mid \lambda_{\phi}(y)\right)=\phi\left(y^{*} x\right)$ for $x, y \in \mathscr{A}$. Let $\mathfrak{S}_{\phi}$ be the Hilbert space which is completion of the pre-Hilbert space $\lambda_{\phi}(\mathscr{A})$. We define a $*$-representation $\pi_{\phi}^{0}$ of $\mathscr{A}$ on $\mathfrak{S}_{\phi}$ by

$$
\pi_{\phi}^{0}(x) \lambda_{\phi}(y)=\lambda_{\phi}(x y)
$$

for $x, y \in \mathscr{A}$. We denote by $\pi_{\phi}$ the closure of $\pi_{\phi}^{0}$. We call the triple $\left(\pi_{\phi}, \lambda_{\phi}, \mathfrak{S}_{\phi}\right)$ the GNS-construction for $\phi$. If $\pi_{\phi}$ is self-adjoint, then $\phi$ is said to be a Riesz functional on $\mathscr{A}$.

\section{§3. Modular Unbounded Left Hilbert Algebras}

In this section we generalize the notion of left Hilbert algebras to the unbounded case, and extend the main results of Tomita to the unbounded case.

Suppose that a $*$-algebra $\mathfrak{U}$ with an involution \# admits an inner product $(\mid)$ satisfying the following condition:

(i) $\quad(\xi \eta \mid \zeta)=\left(\eta \mid \xi^{\# \zeta)}\right.$ for $\xi, \eta, \zeta \in \mathfrak{U}$.

Let $\mathfrak{S}$ be the Hilbert space obtained by completion of $\mathfrak{A}$. By (i) a $*$-representation $\pi_{0}$ of $\mathfrak{U}$ in $\mathfrak{S}$ is defined by $\pi_{0}(\xi) \eta=\xi \eta$ for $\xi, \eta \in \mathfrak{U}$ whose closure is denoted by $\pi_{\mathfrak{M}}$ (simply $\pi$ ). Further, suppose $\mathfrak{U}$ satisfies the following conditions: 
(ii) $\mathfrak{A}^{2}$ is dense in $\mathfrak{A}$, where $\mathfrak{A}^{2}$ denotes the vector space generated by $\{\xi \eta ; \xi, \eta \in \mathfrak{R}\}$;

(iii) $\pi(\mathfrak{H})^{\prime}$ is a von Neumann algebra.

Let $\hat{\pi}_{\mathscr{U}}(\operatorname{simply}, \hat{\pi})$ be the closure of a $*$-representation $\pi_{1}$ of $\mathfrak{A}$ defined by:

$$
\pi_{1}(\xi)\left(\sum_{i=1}^{n} C_{i} x_{i}\right)=\sum_{i=1}^{n} C_{i} \pi(\xi) x_{i} \quad \text { for }\left\{C_{i}\right\} \subset \pi(\mathfrak{R})^{\prime},\left\{x_{i}\right\} \subset \mathscr{D}(\pi) .
$$

Then $\hat{\pi}$ is the smallest closed $*$-representation of $\mathscr{A}$ such that $\pi \subset \hat{\pi} \subset \pi^{*}, \hat{\pi}(\mathfrak{R})^{\prime}=\pi(\mathfrak{H})^{\prime}$ and $\hat{\pi}(\mathfrak{H})^{\prime} \mathscr{D}(\hat{\pi})=\mathscr{D}(\hat{\pi}) \quad$ [16]. We now define a commutant $\mathfrak{X}_{w}^{\prime}$ of $\mathfrak{X}$. For each $\eta \in \mathscr{D}(\hat{\pi})$ we define a linear operator $\pi_{0}^{\prime}(\eta)$ in $\mathscr{K}$ by

$$
\pi_{0}^{\prime}(\eta) \xi=\hat{\pi}(\xi) \eta, \quad \xi \in \mathfrak{R} .
$$

We denote by $\mathscr{D}(\mathfrak{R})^{b}$ the set of all elements $\eta$ of $\mathscr{D}(\hat{\pi})$ such that there exists another element $\eta^{b}$ of $\mathscr{D}(\hat{\pi})$ satisfying the equation:

$$
\left(\eta \mid \xi_{1} \xi_{2}\right)=\left(\xi_{2}^{\#} \xi_{1}^{\#} \mid \eta^{b}\right)
$$

for each $\xi_{1}, \xi_{2} \in \mathfrak{A}$. Since $\mathfrak{U}^{2}$ is dense in $\mathfrak{F}$ by (ii), it follows that $\eta \rightarrow \eta^{b}$ is a conjugate linear operator on $\mathscr{D}(\mathfrak{R})^{b}$ satisfying $\left(\eta^{b}\right)^{b}=\eta$ and $\overline{\pi_{0}^{\prime}\left(\eta^{b}\right)}$ $\subset \pi_{0}^{\prime}(\eta)^{*}$. Further, we note that the closure $\pi_{\mathfrak{r}}^{\prime}(\eta)$ (simply, $\pi^{\prime}(\eta)$ ) of $\pi_{0}^{\prime}(\eta)$ is contained in the unbounded commutant $\pi_{0}(\mathfrak{I})_{\sigma}^{\prime}$ of the $\mathrm{O}_{p}^{*-}$ algebra $\pi_{0}(\mathfrak{H})$. We now define a commutant $\mathfrak{N}_{w}^{\prime}$ (simply, $\left.\mathfrak{X}^{\prime}\right)$ as follows:

$$
\mathfrak{A}_{w}^{\prime}=\left\{\eta \in \mathscr{D}(\mathfrak{R})^{b} ; \pi^{\prime}(\eta) \in \mathscr{B}(\mathfrak{S})\right\} .
$$

Then it is easily shown that $\mathfrak{X}^{\prime}$ is a *-algebra equipped with the multiplication $\eta_{1} \eta_{2}=\pi^{\prime}\left(\eta_{2}\right) \eta_{1}$ and the involution $\eta \rightarrow \eta_{1}^{b}$, and $\pi^{\prime}$ is an anti-*-isomorphism of $\mathfrak{A}^{\prime}$ into the von Neumann algebra $\pi(\mathfrak{U})^{\prime}$ 。

Definition 3. 1. If a *-algebra $\mathscr{A}$ with involution \# admits an inner product satisfying conditions (i), (ii), (iii) and the following conditions (iv), (v), then $\mathfrak{A}$ is said to be an unbounded left Hilbert algebra in $\mathfrak{S}$ : I;

(iv) $\left(\mathfrak{I}^{\prime}\right)^{2}$ is dense in the Hilbert space $\mathfrak{S}$ obtained by completion of

(v) $\mathscr{A} \neq \mathscr{Q}_{\mathrm{b}} \equiv\{\xi \in \mathscr{Y} ; \overline{\pi(\xi)} \in \mathscr{B}(\mathfrak{S})\}$.

Suppose $\mathfrak{A}$ is an unbounded left Hilbert algebra in $\mathfrak{S}$. Then $\mathfrak{X}^{\prime}$ is a right Hilbert algebra in $\mathfrak{S}$. We denote by $F_{\mathfrak{Q}}^{\prime \prime}$ the closure of the map $\eta \in \mathfrak{Q}^{\prime} \rightarrow \eta^{\mathfrak{b}} \in \mathfrak{Y}^{\prime}$ and denote by $S_{\mathfrak{U}}^{\prime \prime}$ the adjoint of $F_{\mathfrak{q}}^{\prime \prime}$. We see that the 
usual commutant $\left(\mathfrak{Y}_{w}^{\prime}\right)^{\prime}$ (simply, $\mathfrak{X}^{\prime \prime}$ ) of the right Hilbert algebra $\mathfrak{U}^{\prime}$ is an achieved left Hilbert algebra in $\mathfrak{S}$ with involution $\xi \rightarrow S_{\mathfrak{r}}^{\prime \prime} \xi$ whose left von Neumann algebra equals $\pi(\mathfrak{U})^{\prime \prime}$, so that the fundamental theorem of Tomita

$$
\begin{aligned}
& J_{\mathfrak{R}}^{\prime \prime} \pi(\mathfrak{U})^{\prime \prime} J_{\mathfrak{Q}}^{\prime \prime}=\pi(\mathfrak{U})^{\prime} \\
& \Delta_{\mathfrak{Q}}^{\prime \prime i t} \pi(\mathfrak{U}){ }^{\prime \prime} \Delta_{\mathfrak{Q}}^{\prime \prime}-i t=\pi(\mathfrak{U})^{\prime \prime} \\
& \Delta_{\mathscr{U}}^{\prime \prime i t} \pi(\mathfrak{U})^{\prime} \Delta_{\mathscr{U}}^{\prime \prime-i t}=\pi(\mathfrak{U})^{\prime}
\end{aligned}
$$

for all $t \in \mathbb{R}$ is obtained, where $J_{\mathfrak{Q}}^{\prime \prime}$ is the modular conjugation operator of $\mathfrak{U}^{\prime \prime}$ and $\Delta_{\mathfrak{U}}^{\prime \prime}$ is the modular operator of $\mathfrak{U}^{\prime \prime}[32,34]$. In order to extend the above results of Tomita to the $\mathrm{O}_{p}^{*}$-algebra $\pi(\mathfrak{U})$, we introduce the following notion.

Definition 3.2. An unbounded left Hilbert algebra $\mathfrak{U}$ is said to be modular if there exists a subspace $\mathscr{D}$ of $\mathscr{D}(\hat{\pi})$ such that

(1) $\mathfrak{U} \subset \mathscr{D}$;

(2) $\hat{\pi}(\mathfrak{U}) \mathscr{D} \subset \mathscr{D}$;

(3) $\quad \Delta_{\mathscr{\imath}}^{\prime \prime i t} \mathscr{D}=\mathscr{D}$ for all $t \in \mathbb{R}$.

Let $\mathfrak{A}$ be a modular unbounded left Hilbert algebra in $\mathfrak{S}$. We denote by $\mathscr{D}_{\mathscr{T}}$ the subspace of $\mathscr{D}(\hat{\pi})$ generated by $\underset{\mathscr{D} \in \mathscr{T}}{\cup} \mathscr{D}$, where $\mathscr{F}$ is the set of all subspaces of $\mathscr{D}(\hat{\pi})$ satisfying the conditions $(1) \sim(3)$ of Definition 3.2. Then it is clear that $\mathscr{D}_{\mathfrak{U}}$ is the largest element of $\mathscr{F}$.

We show that Question $A$ in Introduction is affirmative for every modular unbounded left Hilbert algebra.

Theorem 3. 3. Suppose 9 is a modular unbounded left Hilbert algebra in $\mathfrak{S}$. Then the following statements hold.

(1) Put

$$
\begin{aligned}
& \mathscr{U}(\mathfrak{U})=\left\{X \in \mathscr{L}^{\uparrow}\left(\mathscr{D}_{\mathfrak{U}}\right) ; \bar{X} \text { is affliated with } \pi(\mathfrak{U})^{\prime \prime}\right\}, \\
& \mathscr{V}(\mathscr{L})=\left\{X \in \mathscr{L}^{\dagger}\left(J_{\mathfrak{U}}^{\prime \prime} \mathscr{D}_{\mathfrak{U}}\right) ; \bar{X} \text { is affliated with } \pi(\mathfrak{U})^{\prime}\right\}
\end{aligned}
$$

Then $\mathscr{U}(\mathfrak{U})$ and $\mathscr{V}(\mathfrak{U})$ are generalized von Neumann algebras satisfying

$$
J_{\mathfrak{U}}^{\prime \prime} \mathscr{U}(\mathfrak{U}) J_{\mathfrak{U}}^{\prime \prime}=\mathscr{V}(\mathfrak{U}) \text {. }
$$

In particular, if $\pi$ is self-adjoint, then $\mathscr{U}(\mathfrak{U})$ and $\mathscr{V}(\mathfrak{H})$ are self-adjoint. 
$\mathscr{U}(\mathfrak{U})$ (resp. $\mathscr{V}(\mathfrak{U})$ ) is said to be the left (resp. right) generalized von Neumann algebra of $\mathfrak{X}$.

(2) Put

$$
\sigma_{t}^{\mathfrak{Q}}(X)=\Delta_{\mathscr{थ}}^{\prime \prime i t} X \Delta_{\mathscr{थ}}^{\prime \prime-i t}
$$

for $X \in \mathscr{U}(\mathfrak{U})$ and $t \in \mathbb{P}_{\mathrm{H}}$. Then $\left\{\sigma_{t}^{\mathfrak{2}}\right\}_{t \in \mathbb{R}}$ is a one-parameter group of automorphisms of $\mathscr{U}(\mathfrak{U})$. A similar statement holds for $\mathscr{V}(\mathfrak{U})$.

Proof. It is clear that the restriction $\hat{\pi}(\mathfrak{A}) / \mathscr{D}_{\mathfrak{T}}$ of the $\mathrm{O}_{p}^{*}$-algebra $\hat{\pi}(\mathfrak{U})$ to $\mathscr{D}_{\mathfrak{U}}$ is an $\mathrm{O}_{\mathfrak{p}}^{*}$-algebra on $\mathscr{D}_{\mathfrak{U}}$ such that

$$
\left(\hat{\pi}(\mathfrak{U}) / \mathscr{D}_{\mathfrak{U}}\right)^{\prime}=\pi(\mathfrak{U})^{\prime} \text {. }
$$

Since $\pi(\mathfrak{U})^{\prime} \mathscr{D}(\hat{\pi})=\mathscr{D}(\hat{\pi})$ and (3.3), it follows that the subspace $\mathscr{D}$ of $\mathscr{D}(\hat{\pi})$ generated by $\pi(\mathfrak{U})^{\prime} \mathscr{D}_{\mathfrak{U}}$ satisfies the conditions $(1) \sim(3)$ of Definition 3.2 ; that is, $\mathscr{D} \in \mathscr{F}$. Since $\mathscr{D}_{\mathfrak{Q}}$ is maximum in $\mathscr{F}$, we have $\pi(\mathfrak{U})^{\prime} \mathscr{D}_{\mathfrak{Q}}=\mathscr{D}_{\mathfrak{Q}}$, which implies

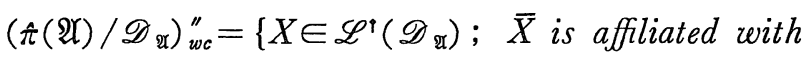

$$
\begin{aligned}
& \left.\left(\hat{\pi}(\mathfrak{U}) / \mathscr{D}_{\mathscr{U}}\right)^{\prime \prime}\right\} \\
& =\mathscr{U}(\mathfrak{U}) \text {. }
\end{aligned}
$$

By Lemma 2.4 $\mathscr{U}(\mathfrak{U})$ is an $\mathrm{O}_{p}^{*}$-algebra on $\mathscr{D}_{\mathfrak{U}}$ with $\mathscr{U}(\mathfrak{U})_{w c}{ }_{w}^{\prime \prime}=\mathscr{U}(\mathfrak{U})$. Since $\Delta_{\mathfrak{\imath}}^{\prime \prime i t} \mathscr{D}_{\mathfrak{U}}=\mathscr{D}_{\mathfrak{\imath}}$ for all $t \in \mathbb{R}$ and (3.3), it follows that

$$
\Delta_{\mathscr{U}}^{\prime \prime i t} \mathscr{U}(\mathfrak{U}) \Delta_{\mathfrak{Q}}^{\prime \prime}-i t=\mathscr{U}(\mathfrak{U})
$$

for all $t \in \mathbb{R}$, which implies that $\left\{\sigma_{t}^{2)}\right\}_{t \in R}$ is a one-parameter group of automorphisms of $\mathscr{U}(\mathfrak{U})$. We show that $\widetilde{\mathscr{D}}_{\mathfrak{U}} \equiv \bigcap_{x \in \mathscr{U}(\mathfrak{U})} \mathscr{D}(\bar{X}) \in \mathscr{F}$. Since

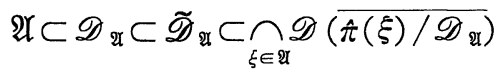

$$
\begin{aligned}
& \subset \bigcap_{\tilde{\xi} \in \mathfrak{\mathscr { I }}} \mathscr{D}(\overline{\hat{\pi}(\xi)})=\mathscr{D}(\hat{\pi}),
\end{aligned}
$$

it follows that $\widetilde{\mathscr{D}}_{\mathfrak{Q}}$ is a subspace of $\mathscr{D}(\hat{\pi})$. For each $x \in \widetilde{\mathscr{D}}_{\mathfrak{V}}$ there is a net $\left\{x_{\alpha}\right\}$ in $\mathscr{D}_{\mathscr{Q}}$ such that $\lim x_{\alpha}=x$ and $\lim X x_{\alpha}=\bar{X} x$ for each $X \in \mathscr{U}(\mathfrak{U})$. Since $\hat{\pi}(\xi) / \mathscr{D}_{\mathscr{U}} \in \mathscr{U}^{\alpha}(\mathfrak{U})$ for each $\xi^{\in} \stackrel{\alpha}{\in}$, we have

$$
\lim _{\alpha} \hat{\pi}(\xi) x_{\alpha}=\hat{\pi}(\xi) x, \quad \lim _{\alpha} X \hat{\pi}(\xi) x_{\alpha}=\overline{X \hat{\pi}(\xi)} x
$$

for each $X \in \mathscr{U}(\mathfrak{R})$, which implies $\hat{\pi}(\mathfrak{U}) \widetilde{\mathscr{D}}_{\mathfrak{Q}} \subset \widetilde{\mathscr{D}}_{\mathfrak{q}}$. Further, we have

$$
\begin{aligned}
& \lim _{\alpha} \Delta_{\mathscr{\ell}}^{\prime \prime i t} x_{\alpha}=\Delta_{\mathscr{\ell}}^{\prime \prime i t} x, \\
& \lim _{\alpha} X \Delta_{\mathfrak{\Omega}}^{\prime \prime i t} x_{\alpha}=\lim _{\alpha} \Delta_{\mathfrak{⿰}}^{\prime \prime i t}\left(\Delta_{\mathfrak{U}}^{\prime \prime-i t} X \Delta_{\mathfrak{\Omega}}^{\prime \prime i t}\right) x_{\alpha} \\
& =\Delta_{\mathfrak{\vartheta}}^{\prime \prime i t}\left(\overline{\Delta_{\mathfrak{थ}}^{\prime \prime-i t} X \Delta_{\mathfrak{\vartheta}}^{\prime \prime i t}}\right) x
\end{aligned}
$$


for each $X \in \mathscr{U}(\mathfrak{U})$ and $t \in \mathbb{R}$, which implies $\Delta_{\mathfrak{Q}}^{\prime \prime i t} \widetilde{\mathscr{D}}_{\mathfrak{Y}} \subset \widetilde{\mathscr{D}}_{\mathfrak{U}}$ for all $t \in \mathbb{R}$. Thus, $\widetilde{\mathscr{D}}_{\mathfrak{T}} \in \mathscr{F}$. Since $\mathscr{D}_{\mathfrak{x}}$ is maximum in $\mathscr{F}$, we have $\widetilde{\mathscr{D}}_{\mathfrak{U}}=\mathscr{D}_{\mathfrak{r}}$, which means that $\mathscr{U}(\mathfrak{U})$ is a closed $\mathrm{O}_{p}^{*}$-algebra on $\mathscr{D}_{\mathfrak{r}}$. Hence $\mathscr{U}(\mathfrak{A})$ is a generalized von Neumann algebra on $\mathscr{D}_{\mathscr{r}}$. Suppose $\pi$ is self-adjoint. Then we can similarly show that $\mathscr{D}_{\mathscr{U}}^{*} \equiv \bigcap_{x \in \mathscr{U}(\mathscr{Q})} \mathscr{D}\left(X^{*}\right) \in \mathscr{F}$, which implies $\mathscr{U}(\mathfrak{U})$ is self-adjoint. It is clear that $J_{\mathfrak{Q}}^{\prime \prime} \mathscr{U}(\mathfrak{U}) J_{\mathfrak{U}}^{\prime \prime}=\mathscr{V}(\mathfrak{U})$. A similar result for $\mathscr{V}(\mathfrak{U})$ holds. This completes the proof.

We next define the notion of standard unbounded left Hilbert algebras. If a $*$-algebra $\mathfrak{A}$ with involution \# admits an inner product satisfying conditions (i), (ii) of Definition 3.1 and the following condition (iv)', then $\mathfrak{A}$ is said to be a generalized left Hilbert algebra.

(iv)' The involution $\xi \rightarrow \xi^{\sharp}$ is closable as a real linear operator on the real pre-Hilbert space $\mathfrak{H}^{2}$.

We note that if $\mathfrak{A}$ is an unbounded left Hilbert algebra then it is a generalized left Hilbert algebra. We consider when the converse holds. Let $\mathfrak{A}$ be a generalized left Hilbert algebra in $\mathfrak{S}$ such that $\pi(\mathfrak{U})^{\prime}$ is a von Neumann algebra. We denote by $S_{\mathfrak{2}}$ the closure of the map: $\xi \in \mathfrak{H}^{2} \rightarrow \xi^{\sharp} \in \mathfrak{H}^{2}$, and put

$$
\mathscr{D}(\mathfrak{U})^{\eta}=\left\{\eta \in \mathscr{D}(\mathfrak{X})^{b} ; \pi^{\prime}(\eta) \text { is affiliated wlth } \pi(\mathfrak{H})^{\prime}\right\} \text {. }
$$

Then $\mathfrak{U}^{\prime} \subset \mathscr{D}(\mathfrak{X})^{n} \subset \mathscr{D}(\mathfrak{X})^{b} \subset \mathscr{D}\left(S_{\mathfrak{U}}^{*}\right)$. It is well-known that in the bounded case $\mathscr{D}(\mathfrak{U})^{\eta}=\mathscr{D}(\mathfrak{A})^{b}=\mathscr{D}\left(S_{\mathfrak{U}}^{*}\right)$, which implies $\left(\mathfrak{U}^{\prime}\right)^{2}$ is dense in the Hilbert space $\mathscr{D}\left(S_{\mathfrak{V}}^{*}\right)$; that is, the converse always holds. But, in the unbounded case $\mathscr{D}(\mathfrak{A})^{\eta} \neq \mathscr{D}(\mathfrak{A})^{b}$ and the converse does not hold in general. It is easily shown by analogy with the bounded case ([32] Lemma 3.3) that when $I \in \overline{\mathbb{\pi}(\mathfrak{U})}^{t_{s}}, \mathscr{D}(\mathfrak{U})^{\eta}$ is dense in $\mathscr{H}$ if and only if $\mathfrak{A}$ is an unbounded left Hilbert algebra in $\mathfrak{S}$, and $\mathscr{D}(\mathfrak{H})^{\eta}$ is dense in the Hilbert space $\mathscr{D}\left(S_{\mathfrak{U}}^{*}\right)$ if and only if $\mathfrak{A}$ is an unbounded left Hilbert algebra in $\mathfrak{S}$ satisfying $S_{\mathfrak{R}}=S_{\mathscr{r}}^{\prime \prime}$

Suppose $\mathfrak{A}$ is a generalized left Hilbert algebra in $\mathfrak{S}_{\mathcal{C}}$. We denote by $S_{\mathfrak{q}}=J_{\mathfrak{r}} \Delta_{\mathfrak{2}}^{1 / 2}$ the polar decomposition of $S_{\mathfrak{q}}$. Then $J_{\mathfrak{r}}$ is a bounded conjugate linear operator on $\mathfrak{S}$ such that $J_{\mathfrak{r}}^{*}=J_{\mathfrak{r}}$ and $J_{\mathfrak{U}}^{2}=I$, and $\Delta_{\mathfrak{U}}$ is a positive self-adjoint operator in $\mathfrak{S}$ such that $\Delta_{\mathfrak{r}}=S_{\mathfrak{r}}^{*} S_{\mathfrak{U}}$ and $\Delta_{\mathfrak{r}}^{-1}=$ $S_{\text {थथ }} S_{\text {थ }}^{*}[8]$.

Suppose $\mathscr{U}$ is an unbounded left Hilbert algebra in $\mathfrak{S}$. Then we note that $S_{\mathscr{\Upsilon}} \subset S_{\mathscr{\Upsilon}}^{\prime \prime}$ however $S_{\mathscr{\Upsilon}}$ and $S_{\mathfrak{I}}^{\prime \prime}$ don't necessarily equal. Hence 
we introduce the following notion.

Definition 3.4. An unbounded left Hilbert algebra $\mathfrak{X}$ is said to be standard if it is modular and $S_{\mathfrak{r}}=S_{\mathscr{\vartheta}}^{\prime \prime}$.

We next consider unbounded left Hilbert algebras induced by positive linear functionals. Let $\mathscr{A}$ be a $*$-algebra with identity $e, \phi$ be a positive linear functional on $\mathscr{A}$ and $\left(\pi_{\phi}, \lambda_{\phi}, \mathfrak{S}_{\phi}\right)$ be the GNSconstruction for $\phi$. If $\lambda_{\phi}(x)=0$ implies $\pi_{\phi}(x)=0$, then the pre-Hilbert space $\lambda_{\phi}(\mathscr{A})$ equipped with the multiplication $\lambda_{\phi}(x) \lambda_{\phi}(y)=\lambda_{\phi}(x y)$ and the involution $\lambda_{\phi}(x)^{\#}=\lambda_{\phi}\left(x^{*}\right)$, becomes a $*$-algebra satisfying conditions (i), (ii). Then we have $\pi\left(\lambda_{\phi}(\mathscr{A})\right)=\pi_{\phi}(\mathscr{A}), \mathscr{D}\left(\lambda_{\phi}(\mathscr{A})\right)^{b} \subset \pi_{\phi}^{0}(\mathscr{A})_{\sigma}^{\prime} \lambda_{\phi}(e)$ $\left(\mathscr{D}\left(\lambda_{\phi}(\mathscr{A})\right)^{b}=\pi_{\phi}^{0}(\mathscr{A}){ }_{\sigma}^{\prime} \lambda_{\phi}(e)\right.$ if $\phi$ is a Riesz functional) and $\lambda_{\phi}(\mathscr{A})^{\prime}$

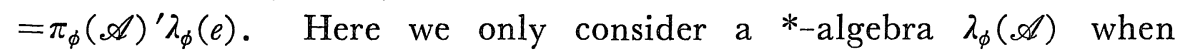
$\mathscr{A} \neq \mathscr{A}_{b}(\phi) \equiv\left\{x \in \mathscr{A} ; \overline{\pi_{\phi}(x)} \in \mathscr{B}\left(\mathscr{S}_{\phi}\right)\right\}$. We note that if $\pi_{\phi}^{0}(\mathscr{A})_{0}^{\prime} \lambda_{\phi}(e)$ is dense in $\mathfrak{S}_{\phi}$ then $\lambda_{\phi}(\mathscr{A})$ is a generalized left Hilbert algebra in $\mathfrak{S}_{\phi}$, and $\lambda_{\phi}(\mathscr{A})$ is an unbounded left Hilbert algebra in $\mathscr{S}_{\phi}$ if and only if $\pi_{\phi}(\mathscr{A})^{\prime}$ is a von Neumann algebra and $\pi_{\phi}(\mathscr{A})^{\prime} \lambda_{\phi}(e)$ is dense in $\mathfrak{S}_{\phi}$. We denote by $S_{\phi}\left(\operatorname{resp} . J_{\phi}, \Delta_{\phi}, S_{\phi}^{\prime \prime}, J_{\phi}^{\prime \prime}, \Delta_{\phi}^{\prime \prime}\right)$ the operators $S_{\lambda_{\phi}(\mathscr{A})}$ (resp. $\left.J_{\lambda_{\phi}(\mathscr{A})}, \Delta_{\lambda_{\phi}(\mathscr{A})}, S_{\lambda_{\phi}(\mathscr{A})}^{\prime \prime}, J_{\lambda_{\phi}(\mathscr{A})}^{\prime \prime}, \Delta_{\lambda_{\phi}(\mathscr{A})}^{\prime \prime}\right)$. With the help of ([1] Theorem 1) it is easy to prove the following

Lemma 3.5. Suppose $\lambda_{\phi}(\mathscr{A})$ is a generalized left Hilbert algebra in $\mathfrak{S}_{\phi}$. Then $\lambda_{\phi}(\mathscr{A})$ is an unbounded left Hilbert algebra in $\mathfrak{S}_{\phi}$ satisfying $S_{\phi}=S_{\phi}^{\prime \prime}$ if and only if $J_{\phi} \pi_{\phi}(\mathscr{A})^{\prime \prime} J_{\phi}=\pi_{\phi}(\mathscr{A})^{\prime}$ and $\left(A J_{\phi} A J_{\phi} \lambda_{\phi}(e) \mid \lambda_{\phi}(e)\right) \geqq 0$ for all $A \in \pi_{\phi}(\mathscr{A})^{\prime \prime}$.

Definition 3.6. A positive linear functional $\phi$ on a *-algebra $\mathscr{A}$ with identity is said to be modular (resp. standard) if $\lambda_{\phi}(\mathscr{A})$ is a modular (resp. standard) unbounded left Hilbert algebra in $\mathfrak{S}_{\phi}$.

We investigate positive linear functionals which satisfy the KMScondition with respect to a continuous one-parameter group of *automorphisms of a locally convex *-algebra.

We denote by $A(0,1)$ the set of all complex-valued functions, bounded and continuous on $0 \leqq \operatorname{Im} z \leqq 1$ and analytic in the interior.

Definition 3.7. Let $\mathscr{A}$ be a locally convex $*$-algebra with identity $e$ and 
$\left\{\alpha_{t}\right\}_{t \in R}$ be a continuous one-parameter group of $*$-aulomorphisms of $\mathscr{A} \cdot A$ continuous positive linear functional $\phi$ on $\mathscr{A}$ is said to satisfy a KMScondition with respect to $\left\{\alpha_{t}\right\}$ if for each $x, y \in \mathscr{A}$ there exists a function $f_{x, y}$ in $A(0,1)$ such that

$$
f_{x, y}(t)=\phi\left(\alpha_{t}(x) y\right), \quad f_{x, y}(t+i)=\phi\left(y \alpha_{t}(x)\right)
$$

for all $t \in \mathbb{R}$. Such a system $\left(\mathscr{A},\left\{\alpha_{t}\right\}, \phi\right)$ is said to be a KMS-system. A KMS-system $\left(\mathscr{A},\left\{\alpha_{t}\right\}, \phi\right)$ is said to be modular (resp. standard) if $\phi$ is modular (resp. standard).

Lemma 3.8. Suppose $\left(\mathscr{A},\left\{\alpha_{t}\right\}, \phi\right)$ is a KMS-system. Then $\lambda_{\phi}(\mathscr{A})$ is a generalized left Hilbert algebra in $\mathfrak{S}_{\phi}$ satisfying $\lambda_{\phi}\left(\alpha_{t}(x)\right)=\Delta_{\phi}^{i t} \lambda_{\phi}(x)$ for all $x \in \mathscr{A}$ and $t \in \mathbb{R}$, and further $\left(\mathscr{A},\left\{\alpha_{t}\right\}, \phi\right)$ is a standard system if and only if $J_{\phi} \pi_{\phi}(\mathscr{A})^{\prime \prime} J_{\phi}=\pi_{\phi}(\mathscr{A})^{\prime}$ and $\left(A J_{\phi} A J_{\phi} \lambda_{\phi}(e) \mid \lambda_{\phi}(e)\right) \geqq 0$ for all $A \in \pi_{\phi}(\mathscr{A})^{\prime \prime}$.

Proof. Since $\phi$ is $\left\{\alpha_{t}\right\}$-invariant and $\phi$ and $\left\{\alpha_{t}\right\}$ are continuous, there exists a strongly continuous one-parameter unitary group $\left\{U_{t}\right\}$ such that $U_{t} \lambda_{\phi}(x)=\lambda_{\phi}\left(\alpha_{t}(x)\right)$ for all $x \in \mathscr{A}$ and $t \in \mathbb{R}$. Since $\phi$ satisfies the KMS-condition with respect to $\left\{\alpha_{t}\right\}$, it is easy to show that $\lambda_{\phi}(\mathscr{A})$ is a $*$-algebra equipped with the multiplication $\lambda_{\phi}(x) \lambda_{\phi}(y)=\lambda_{\phi}(x y)$ and the involution $\lambda_{\phi}(x) \rightarrow \lambda_{\phi}\left(x^{*}\right)$. Suppose that $\lim _{n \rightarrow \infty} \lambda_{\phi}\left(x_{n}\right)=0$ and $\lim _{n \rightarrow \infty} \lambda_{\phi}\left(x_{n}^{*}\right)=\xi$. For each $y \in \mathscr{A}$ we have

$$
\begin{aligned}
& \lim _{n \rightarrow \infty} \sup _{t \in R} f_{x_{n^{\prime}}}(t)=\left(\lambda_{\phi}(y) \mid U_{t} \xi\right), \\
& \lim _{n \rightarrow \infty} \sup _{t \in R} f_{x_{n^{\prime}}, y}(t+i)=0
\end{aligned}
$$

for all $t \in \mathbb{R}$, and hence there exists an element $f$ of $A(0,1)$ such that $f(t)=\left(\lambda_{\phi}(y) \mid U_{t} \xi\right)$ and $f(t+i)=0$ for all $t \in$ 肱. Hence, $\xi=0$. Thus $\lambda_{\phi}(\mathscr{A})$ is a generalized left Hilbert algebra in $\mathscr{S}_{\phi .}$ We denote by $\mathscr{K}_{\phi}$ the closure of $\lambda_{\phi}\left(\mathscr{A}_{h}\right)$, where $\mathscr{A}_{h}=\left\{x \in \mathscr{A} ; x^{*}=x\right\}$. Then $\mathscr{K}_{\phi}$ is a closed real subspace of $\mathscr{S}_{\phi}$ such that $\mathscr{K}_{\phi}+i \mathscr{K}_{\phi}$ is dense in $\mathscr{S}_{\phi}$. Further, since the involution $\lambda_{\phi}(x) \rightarrow \lambda_{\phi}\left(x^{*}\right)$ is closable, we have $\mathscr{K}_{\phi} \cap i \mathscr{K}_{\phi}=\{0\}$, which implies that $S_{\phi}$ equals a closed operator $S$ in $\mathscr{S}_{\phi}$ defined by: $S(\xi+\eta)=\xi-\eta$ for $\xi \in \mathscr{K}_{\phi}$ and $\eta \in i \mathscr{K}_{\phi}$. It hence follows from ([25] Proposition 3.7) that the one-parameter unitary group $\left\{\Delta_{\phi}^{i t}\right\}$ satisfies the KMS-condition with respect to $\mathscr{K}_{\phi}$ in the sense of ([25] Definition 3.4) such that $\Delta_{\phi}^{i t} \mathscr{H}_{\phi}=\mathscr{K}_{\phi}$ for all $t \in \mathbb{R}$. It 
is clear that $U_{t} \mathscr{K}_{\phi}=\mathscr{K}_{\phi}$ for all $t \in \mathbb{R}$. Further, for each $\xi, \eta \in \mathscr{K}_{\phi}$ we have

$$
\begin{aligned}
& \lim \sup _{n-\infty}\left|f_{x_{n}, y_{n}}(t)-\left(\eta \mid U_{t} \xi\right)\right|=0, \\
& \lim \sup _{n \rightarrow \infty}\left|f_{x_{n}, y_{n}}(t+i)-\left(U_{t} \xi \mid \eta\right)\right|=0
\end{aligned}
$$

for all $t \in \mathbb{R}$, where $\left\{x_{n}\right\},\left\{y_{n}\right\} \subset \mathscr{A}_{h}$, which implies that $\left\{U_{t}\right\}$ satisfies the KMS-condition with respect to $\mathscr{K}_{\phi}$. It hence follows from ([25] Theorem 3.8) that $U_{t}=\Delta_{\phi}^{i t}$ for all $t \in \boldsymbol{R}$. The rest follows from the above result and Lemma 3.5. This completes the proof.

We next show that a standard system is constructed by every modular positive linear functional on a $*$-algebra.

Let $\phi$ be a modular positive linear functional on a $*$-algebra $\mathscr{A}$ with identity $e$. Put

$$
\begin{aligned}
& \mathscr{D}_{\phi}=\mathscr{D}_{\lambda_{\phi}(\mathscr{A})} ; \\
& \mathscr{U}\left(\lambda_{\phi}(\mathscr{A})\right)=\left\{X \in \mathscr{L}^{\dagger}\left(\mathscr{D}_{\phi}\right) ; \quad \bar{X} \text { is affiliated with } \pi_{\phi}(\mathscr{A})^{\prime \prime}\right\} ; \\
& \sigma_{t}^{\phi}(X)=\Delta_{\phi}^{\prime \prime i t} X \Delta_{\phi}^{\prime \prime-i t}, \quad X \in \mathscr{U}\left(\lambda_{\phi}(\mathscr{A})\right), \quad t \in \boldsymbol{R} ; \\
& \omega_{\phi}(X)=\left(X \lambda_{\phi}(e) \mid \lambda_{\phi}(e)\right), \quad X \in \mathscr{U}\left(\lambda_{\phi}(\mathscr{A})\right) .
\end{aligned}
$$

Then $\mathscr{U}\left(\lambda_{\phi}(\mathscr{A})\right)$ is a locally convex $*$-algebra equipped with the weak topology, $\left\{\sigma_{t}^{\phi}\right\}$ is a continuous one-parametor group of automorphisms of $\mathscr{U}\left(\lambda_{\phi}(\mathscr{A})\right)$ by Theorem 3.3 and $\omega_{\phi}$ is a continuous positive linear functional on $\mathscr{U}\left(\lambda_{\phi}(\mathscr{A})\right)$.

Theorem 3.9. (1) Suppose $\phi$ is a modular positive linear functional on a *-algebra $\mathscr{A}$ with identity e. Then $\left(\mathscr{U}\left(\lambda_{\phi}(\mathscr{A})\right),\left\{\sigma_{t}^{\phi}\right\}, \omega_{\phi}\right)$ is a standard system.

(2) Suppose $\left(\mathscr{U}\left(\lambda_{\phi}(\mathscr{A})\right),\left\{\alpha_{t}\right\}, \omega_{\phi}\right)$ is a standard system. Then $\alpha_{t}=\sigma_{t}^{\phi}$ for all $t \in \mathbb{R}$.

Proof. (1) It follows from ([32] Theorem 13.1) that for each $A, B \in \pi_{\phi}(\mathscr{A})^{\prime \prime}$ there exists an element $f_{A, B}$ of $A(0,1)$ such that

$$
\begin{aligned}
& f_{A, B}(t)=\left(\Delta_{\phi}^{\prime \prime}-i t B \lambda_{\phi}(e) \mid A^{*} \lambda_{\phi}(e)\right), \\
& f_{A, B}(t+i)=\left(\Delta_{\phi}^{\prime i t} A \lambda_{\phi}(e) \mid B^{*} \lambda_{\phi}(e)\right)
\end{aligned}
$$

for all $t \in \mathbb{R}$. Take arbitary $X, Y \in \mathscr{U}\left(\lambda_{\phi}(\mathscr{A})\right)$. Since $\bar{X}$ and $\bar{Y}$ are affiliated with $\pi_{\phi}(\mathscr{A})^{\prime \prime}$, we have 


$$
\begin{aligned}
& \lim \sup _{n \rightarrow \infty}\left|f_{A_{n^{\prime}, B_{n}}}(t)-\omega_{\phi}\left(\sigma_{t}^{\phi}(X) Y\right)\right|=0, \\
& \lim _{n \rightarrow \infty} \sup _{t \in R}\left|f_{A_{n^{\prime}, B_{n}}}(t+i)-\omega_{\phi}\left(Y \sigma_{t}^{\phi}(X)\right)\right|=0
\end{aligned}
$$

for sequences $\left\{A_{n}\right\},\left\{B_{n}\right\}$ in $\pi_{\phi}(\mathscr{A})^{\prime \prime}$, which implies that there exists an element $f_{X, Y}$ of $A(0,1)$ such that

$$
f_{X, Y}(t)=\omega_{\phi}\left(\sigma_{t}^{\phi}(X) Y\right), f_{X, Y}(t+i)=\omega_{\phi}\left(Y \sigma_{t}^{\phi}(X)\right)
$$

for all $t \in \mathbb{R}$. Hence, $\left(\mathscr{U}\left(\lambda_{\phi}(\mathscr{A})\right),\left\{\sigma_{t}^{\phi}\right\}, \omega_{\phi}\right)$ is a KMS-system. It is clear that $\left(\mathscr{U}\left(\lambda_{\phi}(\mathscr{A})\right),\left\{\sigma_{t}^{\phi}\right\}, \omega_{\phi}\right)$ is a modular system. Further, it follows from Lemma 3.8 that $\lambda_{\omega_{\phi}}\left(\sigma_{t}^{\phi}(X)\right)=\Delta_{\omega_{\phi}}^{i t} \lambda_{\omega_{\phi}}(X)$ for all $X \in$ $\mathscr{U}\left(\lambda_{\phi}(\mathscr{A})\right)$ and $t \in \mathbb{R}$, which implies that $\Delta_{\omega_{\phi}}^{\prime \prime}=\Delta_{\omega_{\phi}}=U \Delta_{\phi}^{\prime \prime} U^{*}$, where $U$ is the unitary operator obtained by $U X \lambda_{\phi}(e)=\lambda_{\omega_{\phi}}(X)$ for $X \in \mathscr{U}\left(\lambda_{\phi}(\mathscr{A})\right)$. Hence, $\left(\mathscr{U}\left(\lambda_{\phi}(\mathscr{A})\right),\left\{\sigma_{t}^{\phi}\right\}, \omega_{\phi}\right)$ is a standard system.

(2) This follows from Lemma 3.8.

\section{§4. Standard Uunbounded Lef Hilbert Algebras Constructed by von Neumann Algebras with Cyclic and Separating Vector}

In this section we consider Question B in Introduction for von Neumann algebras with a cyclic and separating vector.

Let $\mathscr{M}_{0}$ be a von Neumann algebra on a Hilbert space $\mathfrak{S}$ with a cyclic and separating vector $\xi_{0}$. Then $\mathscr{M}_{0} \xi_{0}$ is an achieved left Hilbert algebra in $\mathfrak{S}$ equipped with the multiplication $\left(A \xi_{0}\right)\left(B \xi_{0}\right)$ $=A B \xi_{0}$ and the involution $\left(A \xi_{0}\right)^{\#}=A^{*} \xi_{0}$. Let $\Delta$ be the modular operator of $\mathscr{M}_{0} \xi_{0}, J$ be the modular conjugation operator of $\mathscr{M}_{0} \xi_{0}$ and $\mathscr{M}_{0}^{\sigma}$ be the fixed-point algebra of the modular automorphism group $\left\{\sigma_{t}\right\}$ of $\mathscr{M}_{0} \xi_{0}$ in $\mathscr{M}_{0}$; that is, $\mathscr{M}_{0}^{\sigma}=\left\{A \in \mathscr{M}_{0} ; \sigma_{t}(A) \equiv \Delta^{i t} A \Delta^{-i t}=A\right.$ for all $t \in \mathbb{R}\}$. In this section we construct some $\mathrm{O}_{p}^{*}$-algebras $\mathscr{M}$ such that $\mathscr{M} \xi_{0}$ are standard unbounded left Hilbert algebras in $\mathfrak{K}$ satisfying $\left(\mathscr{M} \xi_{0}\right)^{\prime \prime}=\mathscr{M}_{0} \xi_{0}$.

Theorem 4.1. Suppose $\mathscr{M}_{0}$ is a von Neumann algebra on a Hilbert space $\mathfrak{S}$ with a cyclic and separating vector $\xi_{0}$ and $\mathscr{D}$ is a dense subspace in $\mathfrak{S}$ satisfying

(i) $\xi_{0} \in \mathscr{D}$;

(ii) $\Delta^{i t} \mathscr{D}=\mathscr{D}$ for all $t \in \mathbb{R}$; 
(iii) $\left(\mathscr{M}_{0}^{\mathscr{D}}\right)^{\prime \prime}=\mathscr{M}_{0}$, where $\mathscr{M}_{0}^{\mathscr{D}}=\left\{A / \mathscr{D} ; A \in \mathscr{M}_{0}, A \mathscr{D} \subset \mathscr{D}\right.$ and $\left.A^{*} \mathscr{D} \subset \mathscr{D}\right\} ;$

(iv) $\mathscr{M}_{0}^{\mathscr{D}} \neq{\overline{\mathscr{M}_{0}^{\mathscr{T}}}}_{\mathrm{s}}^{*}$.

Then $\overline{\mathscr{M}}_{0}^{\mathscr{D}}{ }^{*} \xi_{0}$ is a standard unbounded left Hilbert algebra in $\mathfrak{S}$ equipped with the multiplication $\left(X \xi_{0}\right)\left(Y \xi_{0}\right)=X Y \xi_{0}$ and the involution $\left(X \xi_{0}\right)^{\#}=X^{\dagger} \xi_{0}$ such that $\left.\overline{\left(\mathscr{M}_{0}^{D^{t}}{ }^{*} \xi_{0}\right.}\right)^{\prime \prime}=\mathscr{M}_{0} \xi_{0}, \mathscr{D}{\overline{\mathscr{M}_{0}^{\mathscr{D}}}}_{t_{s}^{*} \xi_{0}}=\mathscr{D}(\hat{\pi}), \Delta^{i t} \hat{\pi}\left({\overline{\mathscr{M}_{0}^{\mathscr{D}}}}^{t^{*}} \xi_{0}\right) \Delta^{-i t}=\hat{\pi}\left({\overline{\mathscr{M}_{0}^{\mathscr{D}}}}^{t^{*}} \xi_{0}\right)$ for all $t \in \mathbb{R}$ and the left generalized von Neumann algebra $\mathscr{U}\left(\overline{\mathscr{M}}_{0}^{\mathscr{D}^{*}}{ }^{*} \xi_{0}\right)$ equals ${\overline{\mathscr{M}_{0}^{\mathscr{D}(\hat{\pi})}}}^{t^{*}}={\overline{\mathscr{M}_{0}^{\mathscr{D}}(\hat{\pi})^{t}}}^{t}$, where $\hat{\pi}$ is a closed $*_{\text {-representation }} \hat{\pi}_{\overline{\mathscr{M}}_{0}^{\mathscr{D}} t_{\xi_{0}}^{*}}$ of ${\overline{\mathscr{M}_{0}^{\mathscr{D}}}}^{t^{*}} \xi_{0}$ induced by the left regular representation $\pi \equiv \pi_{\mathscr{M}_{0}^{\mathscr{D}} t_{\xi_{0}}^{*}}$ of ${\overline{\mathscr{M}_{0}^{\mathscr{D}}}}^{t^{*}} \xi_{0}$.

Proof. By (i) and (iii) it is easily shown that ${\overline{M_{0}^{\mathscr{D}}}}^{t_{s}^{*}} \xi_{0}$ is a generalized left Hilbert algebra in $\mathscr{S}$ such that $\pi\left(\overline{\mathscr{M}}_{0}^{\mathscr{D}^{t}} \xi_{0}\right)^{\prime}=\mathscr{M}_{0}^{\prime}$. Since ${\overline{\mathscr{M}_{0}^{\mathscr{D}}}}^{t^{*}}=\left(\mathscr{M}_{0}^{\mathscr{D}}\right)_{w c}^{\prime \prime}$ by Lemma 2.4, (6) and (iii), (iv) it follows that $\pi\left({\overline{\mathscr{M}_{0}^{\mathscr{D}}}}^{t^{*}} \xi_{0}\right) \neq \pi\left({\overline{\mathscr{M}_{0}^{\mathscr{D}}}}^{t_{s}^{*}} \xi_{0}\right)_{\mathrm{b}}$. We now show

$$
\left(\overline{\mathscr{M}}_{0}^{\mathscr{D}}{ }^{t^{*}} \xi_{0}\right)^{\prime}=\mathscr{M}_{0}^{\prime} \xi_{0} \text {. }
$$

Take arbitrary $C \in \mathscr{M}_{0}^{\prime}$. Then we have $C \xi_{0}, C^{*} \xi_{0} \in \mathscr{D}(\hat{\pi})$ and $\left(C \xi_{0} \mid X \xi_{0}\right)$ $=\left(X^{\dagger} \xi_{0} \mid C^{*} \xi_{0}\right)$ for all $X \in{\overline{M_{0}^{\mathscr{D}}}}^{t_{s}^{*}}$, and hence $C \xi_{0} \in \mathscr{D}\left({\overline{\mathscr{M}_{0}^{\mathscr{D}}}}^{t_{s}^{*}} \xi_{0}\right)^{b}$. Further, since $\pi^{\prime}\left(C \xi_{0}\right) X \xi_{0}=\hat{\pi}\left(X \xi_{0}\right) C \xi_{0}=C X \xi_{0}$ for all $X \in{\overline{\mathscr{M}_{0}^{O}}}^{t_{s}^{*}}$, it follows that $C \xi_{0} \in\left(\overline{\mathscr{M}}_{0}^{\mathscr{D}^{*}}{ }^{*} \xi_{0}\right)^{\prime}$. Conversely suppose $\eta \in\left({\overline{\mathscr{M}_{0}^{\mathscr{O}}}}^{l^{*}} \xi_{0}\right)^{\prime}$. Then $\pi^{\prime}(\eta) \in$ $\pi\left({\overline{\mathscr{M}_{0}^{\mathscr{D}}}}^{t^{*}} \xi_{0}\right)^{\prime}=\mathscr{M}_{0}^{\prime}$, and so $\eta=\pi^{\prime}(\eta) \xi_{0} \in \mathscr{M}_{0}^{\prime} \xi_{0}$. Thus ${\overline{\mathscr{M}_{0}^{\mathscr{D}}}}^{t^{*}} \xi_{0}$ is an unbounded left Hilbert algebra in $\mathscr{S}$ such that $\left(\overline{\mathscr{M}}_{0}^{\mathscr{O}^{i}}{ }^{*} \xi_{0}\right)^{\prime \prime}=\mathscr{M}_{0} \xi_{0}$ and $S_{\mathscr{M}_{0}^{\mathscr{D}} t_{\xi_{\xi_{0}}}^{*}}^{*}$ equals $S_{\mathscr{M}_{0} \xi_{0}}$.

By (ii) we have $\Delta^{i t}{\overline{\mathscr{M}_{0}^{\mathscr{D}}}}^{t^{*}} \Delta^{-i t}={\overline{\mathscr{M}_{0}^{\mathscr{D}}}}^{t^{*}}$ for all $t \in \mathbb{R}$, and hence

$$
\Delta^{i t}{\overline{M_{0}^{\mathscr{D}}}}^{t^{*}} \xi_{0}={\overline{\mathscr{M}_{0}^{\mathscr{D}}}}^{t^{*}} \xi_{0}
$$

for all $t \in \mathbb{R}$, which implies that $\overline{\mathscr{M}}_{0}^{\mathscr{D}^{*}} \xi_{0}$ is modular. Further, we have

$$
S_{\mathscr{M}_{0} \xi_{0}}=S_{\mathscr{M}_{0}^{\mathscr{O} \xi_{0}}} \subset S_{\mathscr{M}_{0}^{\mathscr{D}} t_{\xi_{0}}^{*}} \subset S_{\mathscr{M}_{0}^{\mathscr{O}}{ }^{\prime \prime}} t_{\xi_{0}}^{*}=S_{\mathscr{H}_{0} \xi_{0}}
$$


which implies that $\overline{\mathscr{M}}_{0}^{\mathscr{D}^{*}} \xi_{0}$ is standard.

We next show

$$
\mathscr{D} \frac{\mathbb{M}_{0}^{\mathscr{D}} t_{s}^{*}}{*}=\mathscr{D}(\hat{\pi}) \text {. }
$$

The statement (4.2) implies $\Delta^{i t} \mathscr{D}(\pi)=\mathscr{D}(\pi)$ and $\pi\left(\Delta^{i t} X \xi_{0}\right)=\Delta^{i t} \pi\left(X \xi_{0}\right) \Delta^{-i t}$ for all $X \in \mathscr{M}$ and $t \in \mathbb{R}$, so that we have

$$
\Delta^{i t}\left(\sum_{i=1}^{n} C_{i} \xi_{i}\right)=\sum_{i=1}^{n}\left(\Delta^{i t} C_{i} \Delta^{-i t}\right) \Delta^{i t} \xi_{i} \in \mathscr{D}\left(\pi_{1}\right)
$$

and

$$
\begin{aligned}
\Delta^{i t} \pi_{1}\left(X \xi_{0}\right) \Delta^{-i t}\left(\sum_{i=1}^{n} C_{i} \xi_{i}\right) & =\Delta^{i t} \pi_{1}\left(X \xi_{0}\right) \sum_{i=1}^{n}\left(\Delta^{-i t} C_{i} \Delta^{i t}\right) \Delta^{-i t} \xi_{i} \\
& =\Delta^{i t} \sum_{i=1}^{n}\left(\Delta^{-i t} C_{i} \Delta^{i t}\right) \pi\left(X \xi_{0}\right) \Delta^{-i t} \xi_{i} \\
& =\sum_{i=1}^{n} C_{i} \Delta^{\Delta t} \pi\left(X \xi_{0}\right) \Delta^{-i t} \xi_{i} \\
& =\sum_{i=1}^{n} C_{i} \pi\left(\Delta^{i t} X \xi_{0}\right) \xi_{i} \\
& =\pi_{1}\left(\Delta^{i t} X \xi_{0}\right)\left(\sum_{i=1}^{n} C_{i} \xi_{i}\right)
\end{aligned}
$$

for all $\sum_{i=1}^{n} C_{i} \xi_{i} \in \mathscr{D}\left(\pi_{1}\right), \quad X \in{\overline{M_{0}^{\mathscr{D}}}}^{t^{*}}$ and $t \in \mathbb{P}$, which implies that $\Delta^{i t} \mathscr{D}(\hat{\pi})=\mathscr{D}(\hat{\pi})$ and $\Delta^{i i} \hat{\pi}\left(X \xi_{0}\right) \Delta^{-i t}=\hat{\pi}\left(\Delta^{i t} X \xi_{0}\right) \quad$ for $\quad$ all $\quad X \in \overline{\mathscr{M}}_{0}^{\mathscr{D}_{s}}{ }^{*}$ and $t \in \mathbb{R}$.

We finally show $\mathscr{U}\left({\overline{\mathscr{M}_{0}^{\mathscr{D}}}}^{t_{s}^{*}} \xi_{0}\right)={\overline{\mathscr{M}_{0}^{\mathscr{D}(\hat{\pi})}}}^{t_{s}^{*}}$. Since

$\mathscr{U}\left({\overline{M_{0}^{\mathscr{D}}}}^{t_{s}^{*}} \xi_{0}\right)=\left\{X \in \mathscr{L}^{\dagger}(\mathscr{D}(\hat{\pi})) ; \bar{X}\right.$ is affliated with $\left.\mathscr{M}_{0}\right\}$

and

$$
\begin{aligned}
{\overline{\mathscr{M}_{0}^{\mathscr{D}}(\hat{\pi})^{t}}}^{t_{w}} & ={\overline{\mathscr{M}_{0}^{\mathscr{D}(\hat{\pi}}}}^{t_{s}^{*}}=\left(\mathscr{M}_{0}^{\mathscr{D}(\hat{\pi})}\right)_{w c}^{\prime \prime} \\
& =\left\{X \in \mathscr{L}^{\dagger}(\mathscr{D}(\hat{\pi})) ; \bar{X} \text { is affiliated with }\left(\mathscr{M}_{0}^{\mathscr{D}(\hat{\pi})}\right)^{\prime \prime}\right\}
\end{aligned}
$$

by (4.3) and Lemma 2.4, (6), it is sufficient to show $\left(\mathscr{M}_{0}^{\mathscr{O}(\hat{\pi})}\right)^{\prime}=\mathscr{M}_{0}^{\prime}$ Take arbitrary $A \in \mathscr{M}_{0}$ with $A / \mathscr{D} \in \mathscr{M}_{0}^{\mathscr{D}}$. Then it is easily shown that $A / \mathscr{D}(\pi) \in \mathscr{M}_{0}^{\mathscr{D}(\pi)}$ and $\pi\left(X \xi_{0}\right) A \xi=\pi\left(X A \xi_{0}\right) \xi$ for all $X \in{\overline{\mathscr{M}_{0}^{\mathscr{D}}}}^{t_{s}^{*}}$ and $\xi \in$ $\mathscr{D}(\pi)$, and hence

$$
A\left(\sum_{i=1}^{n} C_{i} \xi_{i}\right)=\sum_{i=1}^{n} C_{i} A \xi_{i} \in \mathscr{D}\left(\pi_{1}\right),
$$




$$
\begin{aligned}
\pi_{1}\left(X \xi_{0}\right) A\left(\sum_{i=1}^{n} C_{i} \xi_{i}\right) & =\sum_{i=1}^{n} C_{i} \pi\left(X \xi_{0}\right) A \xi_{i} \\
& =\sum_{i=1}^{n} C_{i} \pi\left(X A \xi_{0}\right) \xi_{i} \\
& =\pi_{1}\left(X A \xi_{0}\right)\left(\sum_{i=1}^{n} C_{i} \xi_{i}\right)
\end{aligned}
$$

for each $G_{i} \in \mathscr{M}_{0}^{\prime}$ and $\xi_{i} \in \mathscr{D}(\pi)$, which implies $A / \mathscr{D}(\hat{\pi}) \in \mathscr{M}_{0}^{\mathscr{D}(\hat{\pi})}$. Thus we have $\overline{\mathscr{M}_{0}^{\mathscr{D}}} \equiv\left\{\bar{A} ; \quad A \in \mathscr{M}_{0}^{\mathscr{D}}\right\} \subset \overline{\mathscr{M}_{0}^{\mathscr{D}\left(\pi_{1}\right)}} \subset \overline{\mathscr{M}_{0}^{\mathscr{D}(\hat{\pi})}} \subset \mathscr{M}_{0}$, which implies $\left(\mathscr{M}_{0}^{\mathscr{D}(\hat{\pi})}\right)^{\prime}=\left(\mathscr{M}_{0}^{\mathscr{D}}\right)^{\prime}=\mathscr{M}_{0}^{\prime}$. This completes the proof.

We can prove the following in similar to the proof of Theorem 4. 1 .

Theorem 4.2. Suppose $\mathscr{M}_{0}$ is a von Neumann algebra on a Hilbert space $\mathfrak{S}$ with a cyclic and separating vector $\xi_{0}$ and $\mathscr{D}$ is a dense subspace in $\mathfrak{S}$ satisfying the conditions (i), (ii) and (iii) of Theorem 4.1 and

(iv)' $\mathscr{L}^{\dagger}(\mathscr{D})$ is closed and $\mathscr{M}_{0}^{\mathscr{D}} \neq{\overline{\mathscr{M}_{0}^{\mathscr{D}}}}^{t^{*}}$.

Then $\overline{\mathscr{M}}_{0}^{\mathscr{D}^{*}}{ }^{*} \xi_{0}$ is a standard unbounded left Hilbert algebra in $\mathfrak{S}$ such that

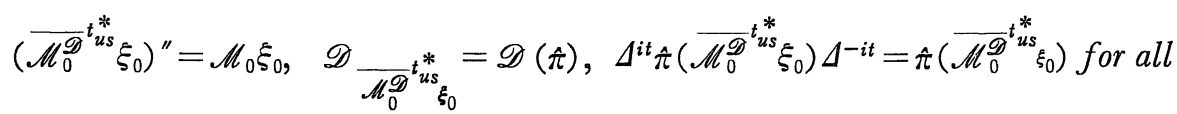
$t \in \boldsymbol{R}$ and $\mathscr{U}\left({\overline{\mathscr{M}_{0}^{\mathscr{D}}}}^{t^{*}} \xi_{0}\right)={\overline{\mathscr{M}_{0}^{\mathscr{D}(\hat{\pi})}}}^{t_{s}^{*}}={\overline{\mathscr{M}_{0}^{\mathscr{D}(\hat{\pi})}}}^{t}$ , where $\hat{\pi}=\hat{\pi}_{{\overline{\mathcal{M}_{0}}}^{\mathscr{D}} t^{*}{ }_{\xi_{0}}^{*}} \cdot$

We give some examples of the systems $\left(\mathscr{M}_{0}, \xi_{0}, \mathscr{D}\right)$ satisfying the conditions (i) $\sim$ (iv), (iv)' of Theorem 4.1 and Theorem 4.2. We first prepare the following lemma without the proof.

Lemma 4.3. Let $T$ be a positive self-adjoint operator in a Hilbert space \$. Put

$$
\mathscr{D}^{\infty}(T)=\bigcap_{n=1}^{\infty} \mathscr{D}\left(T^{n}\right) .
$$

Then $\mathscr{L}^{\dagger}\left(\mathscr{D}^{\infty}(T)\right)$ is a self-adjoint $O_{p}^{*}$-algebra on $\mathscr{D}^{\infty}(T)$, and the induced topology $t_{\mathscr{L}^{\dagger}\left(\mathscr{D}^{\infty}(T)\right)}$ on $\mathscr{D}^{\infty}(T)$ is generated by a family of seminorms: $\|\xi\|_{n}$ $=\left\|T^{n} \xi\right\|, n \in N \cup\{0\}$, so that $\left(\mathscr{D}^{\infty}(T), t_{\mathscr{L}^{\dagger}\left(\mathscr{D}^{\infty}(T)\right)}\right)$ is a Fréchet space. Further, the ultra-strong topology $t_{u s}^{*}$ on $\mathscr{L}^{\dagger}\left(\mathscr{D}^{\infty}(T)\right)$ is generated by a family of seminorms: $P_{n, \xi}^{*}(X)=\left\|T^{n} X \xi\right\|+\left\|T^{n} X^{\dagger} \xi\right\|$ for $n \in N \cup\{0\}$ and $\xi \in \mathscr{D}^{\infty}(T)$. 
Corollary 4. 4. Suppose $T$ is a positive self-adjoint unbounded operator in $\mathscr{S}$ affiliated with $\mathscr{M}_{0}^{\sigma}$ such that $\xi_{0} \in \mathscr{D}^{\infty}(T)$. Then ${\overline{\mathscr{M O}^{\infty}}}^{{ }^{\infty}(T)^{t}}{ }^{{ }^{*}} \xi_{0}$ and $\overline{\mathscr{M}}_{0}^{\mathscr{O}^{\infty}(T)^{s_{s}^{*}}} \xi_{0}$ are standard unbounded left Hilbert algebras in $\mathfrak{S}$ containing $\left\{T^{n} \xi_{0} ; n \in \mathbb{N}\right\}$ such that

$$
\begin{aligned}
& \left({\overline{\mathscr{M}_{0}^{D^{\infty}(T)}}}^{t_{u s}^{*}} \xi_{0}\right)^{\prime \prime}=\left({\overline{\mathscr{M}_{0}^{O{ }^{\infty}(T)}}}^{t_{s}^{*}} \xi_{0}\right)^{\prime \prime}=\mathscr{M}_{0} \xi_{0}, \\
& \mathscr{D}_{\mathscr{M}_{0}^{\mathscr{D}^{\infty}(T)}{ }^{t_{u \xi_{\xi_{0}}}^{*}}}^{*}=\mathscr{D}{\overline{\mathcal{M}_{0}^{\mathscr{O}^{\infty}(T)}}}_{t_{\xi_{\xi_{0}}}^{*}}=\mathscr{D}^{\infty}(T)
\end{aligned}
$$

and

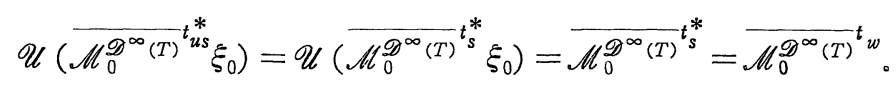

Proof. We first show that $\left(\mathscr{M}_{0}, \xi_{0}, \mathscr{D}^{\infty}(T)\right)$ satisfies the conditions (i) (iv)' of Theorem 4.2. Since $T$ is affiliated with $\mathscr{M}_{0}^{o}$, it follows that $\Delta^{i t} \mathscr{D}^{\infty}(T)=\mathscr{D}^{\infty}(T)$ for all $t \in \mathbb{R}$. Let $T=\int_{0}^{\infty} \lambda d E_{T}(\lambda)$ be the spectral resolution of $T$. Then, for each $A \in \mathscr{M}_{0}$ a sequence $\left\{E_{T}(n)\right.$ $\left.A E_{T}(n) ; n \in \mathbb{N}\right\}$ in $\overline{\mathscr{M}_{0}^{\mathscr{D}}(T)} \equiv\left\{\bar{X} ; X \in \mathscr{M}_{0}^{\mathscr{O}^{\infty}(T)}\right\}$ converges weakly to $A$, and hence $\left(\mathscr{M}_{0}^{\mathscr{O}^{\infty}(T)}\right)^{\prime \prime}=\mathscr{M}_{0} . \quad$ By Lemma 4.3, $\mathscr{L}^{\dagger}\left(\mathscr{D}^{\infty}(T)\right)$ is selfadjoint. Since $T^{n} E_{T}(k) \in \overline{\mathscr{M}_{0}^{\mathscr{O}^{\infty}(T)}}$ for $n, k \in \mathbb{N}$ and $\lim _{k \rightarrow \infty}|| T^{m} T^{n} E_{T}(k) \xi-$ $T^{m} T^{n} \xi \|=0$ for each $\xi \in \mathscr{D}^{\infty}(T)$ and $m \in \mathbb{N}$, it follows from Lemma 4.3 that

$$
T^{n} / \mathscr{D}^{\infty}(T) \in{\overline{\mathscr{M}_{0}^{\infty}(T)}}^{t *}
$$

for $n \in N$, which implies ${\overline{\mathscr{M}_{0}^{\mathscr{D}^{\infty}(T)}}}^{t}{ }^{*} \neq \mathscr{M}_{0}^{\mathscr{O}^{\infty}(T)}$. Thus $\left(\mathscr{M}_{0}, \quad \xi_{0}, \quad \mathscr{D}^{\infty}(T)\right)$ satisfies the conditions (i) $\sim\left(\right.$ iv)' of Theorem 4.2, so that $\overline{\mathscr{M}}_{0}^{\mathscr{D}^{\infty}(T)^{\infty}}{ }^{t} \xi_{0}$ is a standard unbounded left Hilbert algebra in $\mathfrak{S}$ such that

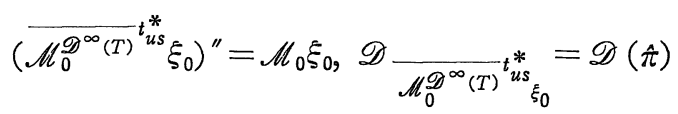

and

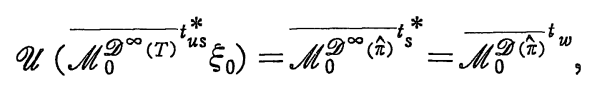

where $\hat{\pi}=\hat{\pi} \hat{\mathscr{M}}_{\mathscr{D}^{\infty}(T)} t_{u_{\xi_{0}}}^{*} \cdot$ Hence, it is sufficient to show $\mathscr{D}(\hat{\pi})=\mathscr{D}^{\infty}(T)$. By (4.4) and Lemma 4.3 we have 


$$
t_{\mathscr{L}^{+}\left(\mathscr{D}^{\infty}(T)\right)}=t_{\mathcal{M}_{0}^{\mathscr{O}^{\infty}(T)}}{ }_{u s}^{*} \cdot
$$

Since a subset $\left\{E_{T}(m) A E_{T}(n) \xi_{0} ; m, n \in \mathbb{N}, A \in \mathscr{M}_{0}\right\}$ of $\mathscr{M}_{0}^{\mathscr{O}^{\infty}(T)} \xi_{0}$ is dense in the Fréchet space $\left(\mathscr{D}^{\infty}(T), t_{\mathscr{L}^{+}\left(\mathscr{D}^{\infty}(T)\right)}\right)$, it follows from (4.5) that ${\overline{\mathscr{M}_{0}^{\infty}(T)}}^{t}{ }^{*}{ }^{*} \xi_{0}$ is dense in $\mathscr{D}^{\infty}(T)$ with respect to the induced topology

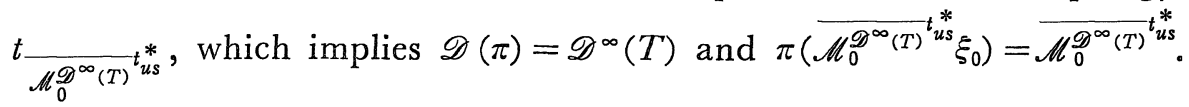
Further, since $\mathscr{D}^{\infty}(T)$ is dense in the Hilbert space $\mathscr{D}\left(T^{n}\right)$ for $n \in \mathbb{N}$, it follows from (4.4) that $\overline{\mathscr{M}}_{0}^{\mathscr{O}^{\infty}(T)}{ }^{t}{ }_{u s}^{*}$ is a self-adjoint $O_{p}^{*}$-algebra on $\mathscr{D}^{\infty}(T)$, which implies $\mathscr{D}(\hat{\pi})=\mathscr{D}(\pi)=\mathscr{D}^{\infty}(T)$.

Similar results for $\overline{\mathscr{M}}_{0}^{\mathscr{O}^{\infty}(T)} t^{*} \xi_{0}$ hold. This completes the proof.

Let $\mathscr{D}$ be a dense subspace of $\mathscr{S}$ and $\mathscr{M}$ be a subset of $\mathscr{C}^{+}(\mathscr{D}, \mathfrak{S})$. We denote by $\overline{\mathscr{M}}^{t_{s}^{*} i_{1} \mathscr{C}^{+}(\mathscr{D}, \mathscr{B})}$ the closure of $\mathscr{M}$ in $\mathscr{C}^{+}(\mathscr{D}, \mathscr{S})$ with respect to the topology $t_{s}^{*}$ generated by a family $\left\{P_{\xi}^{*} ; \xi \in \mathscr{D}\right\}$ of seminorms: $P_{\xi}^{*}(X)=\|X \xi\|+\left\|X^{*} \xi\right\|, X \in \mathscr{C}^{+}(\mathscr{D}, \mathfrak{L})$.

Corollary 4.5. Suppose $T^{\prime}$ is a positive self-adjoint unbounded operator in $\mathscr{S}$ affiliated with $\mathscr{M}_{0}^{\prime \sigma}$ such that $\xi_{0} \in \mathscr{D}^{\infty}\left(T^{\prime}\right)$ and $\overline{\mathscr{M}}_{0}^{t_{s}^{*} \text { in } \mathscr{C}^{+}\left(\mathscr{D}^{\infty}\left(T^{\prime}\right), \mathscr{(}\right)} \neq \mathscr{M}_{0}$.

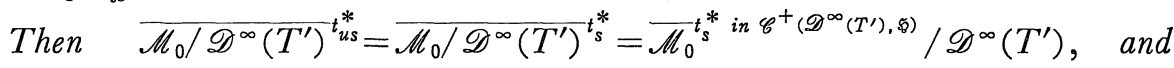
$\bar{M}_{0} / \mathscr{D}^{\infty}\left(T^{\prime}\right)^{t_{s}^{*}} \xi_{0}$ is a standard unbounded left Hilbert algebra in $\mathfrak{H}$ such that $\left(\overline{\mathscr{M}}_{0} / \mathscr{D}^{\infty}\left(T^{\prime}\right)^{t_{s}^{*} \tilde{\xi}_{0}}\right)^{\prime \prime}=\mathscr{M}_{0} \xi_{0}$ and $\mathscr{U}\left(\overline{\mathscr{M}}_{0} / \mathscr{D}^{\infty}\left(T^{\prime}\right)^{t^{*} \xi_{0}}\right)$ equals a closed EW*-algebra $\overline{\mathscr{M}_{0} / \mathscr{D}(\hat{\pi})^{t_{s}^{*}}}$ on $\mathscr{D}(\hat{\pi})$ over $\mathscr{M}_{0}$, where $\hat{\pi}=\hat{\pi} \overline{\bar{M}_{0 / \mathscr{D}^{\infty}\left(T^{\prime}\right)} t_{\xi_{0}}^{*}}$.

Proof. Since $\mathscr{M}_{0} / \mathscr{D}^{\infty}\left(T^{\prime}\right)=\mathscr{M}_{0}^{\mathscr{O}^{\infty}\left(T^{\prime \prime}\right)}$, it follows that $\left(\mathscr{M}_{0}, \xi_{0}, \mathscr{D}^{\infty}\left(T^{\prime}\right)\right)$ satisfies the conditions (i) (iii) of Theorem 4.1. It is clear that

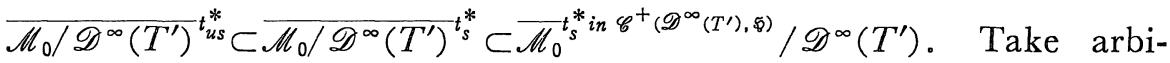
trary $X \in \overline{\mathscr{M}}_{0}^{t_{s}^{*} \text { in } \mathscr{C}^{+}\left(\mathscr{D}^{\infty}\left(T^{\prime}\right), \mathscr{}\right)}$. Then there exists a net $\left\{A_{\alpha}\right\}$ in $\mathscr{M}_{0}$ such that $\lim A_{\alpha} \xi=X \xi$ and $\lim A_{\alpha}^{*} \xi=X^{*} \xi$ for each $\xi \in \mathscr{D}^{\infty}\left(T^{\prime}\right)$. For each $k \in N^{\alpha}$ and $\xi \in \mathscr{D}^{\infty}\left(T^{\prime}\right)$ we have

$$
\lim _{\alpha} T^{\prime k} A_{\alpha} \xi=X T^{\prime k} \xi, \quad \lim _{\alpha} T^{\prime k} A_{\alpha}^{*} \xi=X^{*} T^{\prime k} \xi,
$$

which implies by Lemma 4.3 that $\left\{X_{\alpha}\right\}$ converges to $X$ with respect

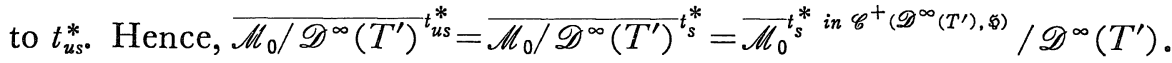


Since $\overline{\mathscr{M}}_{0}^{t_{s}^{*} \text { in } \mathscr{C}^{+}\left(\mathscr{D}^{\infty}\left(T^{\prime}\right), \mathscr{\xi}\right)} \neq \mathscr{M}_{0}$, it follows that $\left(\mathscr{M}_{0}, \xi_{0}, \mathscr{D}^{\infty}\left(T^{\prime}\right)\right)$ satisfies the condition (iv) of Theorem 4.1, so that $\bar{M}_{0} / \mathscr{D}^{\infty}\left(T^{\prime}\right)^{t_{s}^{*}} \xi_{0}$ is a standard unbounded left Hilbert algebra in $\mathscr{S C}_{\mathrm{C}}$ such that $\left(\mathscr{M}_{0} / \mathscr{D}^{\infty}\left(T^{\prime}\right)^{t_{s}^{*}} \xi_{0}\right)^{\prime \prime}=\mathscr{M}_{0} \xi_{0}$ and $\mathscr{U}\left(\overline{\mathscr{M}}_{0} / \mathscr{D}^{\infty}\left(T^{\prime}\right)^{t_{s}^{*}} \xi_{0}\right)=\overline{\mathscr{M}}_{0}^{\mathscr{D}(\hat{\pi})} t_{s}^{*}$. Further, it is easy to show $\mathscr{M}_{0}^{\mathscr{O}(\hat{\pi})}=$ $\mathscr{M}_{0} / \mathscr{D}(\hat{\pi})$, which implies our assertion.

By Corollary 4.4 and Corollary 4.5 we have the following

Corollary 4.6. Suppose $T$ is a positive self-adjoint unbounded operator in $\mathfrak{S}$ affliated with $\mathscr{M}_{0} \cap \mathscr{M}_{0}^{\prime}$ such that $\xi_{0} \in \mathscr{D}^{\infty}(T)$. Then

$$
\overline{\mathscr{M}}_{0} / \mathscr{D}^{\infty}(T)^{t_{u s}^{*}}=\overline{\mathscr{M}}_{0} / \mathscr{D}^{\infty}(T)^{t_{s}^{*}}=\left\{X \in \mathscr{L}^{+}\left(\mathscr{D}^{\infty}(T)\right) ;\right.
$$

$\bar{X}$ is affliated with $\left.\mathscr{M}_{0}\right\}$, which is a closed $E W^{*}$-algebra on $\mathscr{D}^{\infty}(T)$ over $\mathscr{M}_{0}$. Further, $\overline{\mathscr{M}}_{0} / \mathscr{D}^{\infty}(T)^{t_{s}^{*}} \xi_{0}$ is a standard unbounded left Hilbert algebra in $\mathfrak{S}$ such that

$$
\left(\overline{\mathscr{M}_{0} / \mathscr{D}^{\infty}(T)^{t}}{ }^{*} \xi_{0}\right)^{\prime \prime}=\mathscr{M}_{0} \xi_{0}, \mathscr{D}_{\bar{M}_{0}\left(\mathscr{D}^{\infty}(T)\right.} t_{s \xi_{0}}^{*}=\mathscr{D}^{\infty}(T)
$$

and

$$
\mathscr{U}\left(\overline{\mathscr{M}}_{0} / \mathscr{D}^{\infty}(T)^{t_{s}^{*}} \xi_{0}\right)=\overline{\mathscr{M}}_{0} / \mathscr{D}^{\infty}(T)^{t^{*}}=\overline{\mathscr{M}}_{0} / \mathscr{D}^{\infty}(T)^{t}{ }^{w_{0}} \text { 。 }
$$

We denote by $T\left(\mathscr{M}_{0}\right)$ the von Neumann subalgebra $\left\{A \in \mathscr{M}_{0}\right.$; $A \xi_{0} \in \mathscr{D}_{R}(\Delta) \equiv \bigcap_{t \in R} \mathscr{D}\left(\Delta^{t}\right)$ and $\Delta^{\alpha} A \xi_{0} \in \mathscr{M}_{0} \xi_{0}$ for all $\left.\alpha \in \mathbb{C}\right\}$ of $\mathscr{M}_{0}$. Then $T\left(\mathscr{M}_{0}\right) \xi_{0}$ is a maximal Tomita algebra in $\mathscr{S}_{\mathcal{E}}$ equivalent to $\mathscr{M}_{0} \xi_{0}$, and similar results to Lemma 4.3 for $\mathscr{D}_{k}(\Delta)$ hold. Hence, we can prove the following in the same way as in Corollary 4.4 and Corollary 4. 5 .

Corollary 4.7. Suppose $\Delta$ is not bounded and $\overline{\mathscr{M}}_{0}^{t_{s}^{*} \text { in } \mathscr{C}^{+}\left(\mathscr{D}_{R}(\Delta) \text {, }\right)} \neq \mathscr{M}_{0}^{\sigma}$

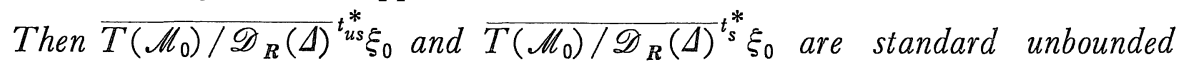
left Hilbert algebras is $\mathfrak{S}$ satisfying

$$
\Delta^{\alpha}{\overline{T\left(\mathscr{M}_{0}\right) / \mathscr{D}_{R}(\Delta)}}^{t_{u s}^{*}} \Delta^{-\alpha}=\overline{T\left(\mathscr{M}_{0}\right) / \mathscr{D}_{R}(\Delta)^{t}{ }^{*}}
$$

and

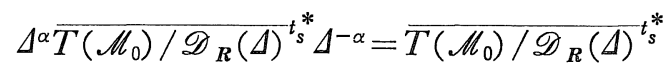

for all $\alpha \in \mathbb{C}$. 
The following result is an answer to Question B in Introduction.

Theorem 4.8. Let $\mathscr{M}_{0}$ be a von Neumann algebra on a Hilbert space $\mathfrak{S}$ with a cyclic and separating vector $\xi_{0}$, and $\mathscr{M}_{0}^{o}$ be the fixed point algebra of the modular automorphism group $\left\{\sigma_{t}\right\}$ of $\mathscr{M}_{0} \xi_{0}$ in $\mathscr{M}_{0}$. Suppose $\mathscr{M}_{0}^{\circ}$ is infinitely dimensional. Then there exist standard unbounded left Hilbert algebras in $\mathfrak{S}$ whose double commutant equals $\mathscr{M}_{0} \xi_{0}$.

Proof. Since $\mathscr{M}_{0}^{\sigma}$ is infinitely dimensional, there exists a sequence of mutually orthogonal projections $\left\{E_{n}\right\}$ in $\mathscr{M}_{0}^{o}$ such that $\left\|E_{n} \xi_{0}\right\|<1$ and $\log \left\|E_{n} \xi_{0}\right\|-\log \left\|E_{n+1} \xi_{0}\right\|>1$ for $n \in \mathbb{N}$. Then it is easily shown that $T=\sum_{n=1}^{\infty}\left(-\log \left\|E_{n} \xi_{0}\right\|_{1}\right) E_{n}$ is a positive self-adjoint unbounded operator affiliated with $\mathscr{M}_{0}^{o}$ such that $\xi_{0} \in \mathscr{D}^{\infty}(T)$. Hence, our assersion holds by Corollary 4.4 .

Corollary 4.9. Suppose $\mathscr{M}_{0}$ is a semifinite von Neumann algebra on a Hilbert space $\mathfrak{S E}_{\mathrm{g}}$ with a cyclic and separating vector $\xi_{0}$ and the spectrum $S_{p}(\Delta)$ of the modular operator $\Delta$ is an infinite set. Then there exist standard unbounded left Hilbert algebras in $\mathfrak{S}$ whose double commutant equals $\mathscr{M}_{0} \xi_{0}$.

Proof. By ([32] Theorem 14.2) there exists a positive self-adjoint operator $K$ affiliated with $\mathscr{M}_{0}$ such that $\Delta=K^{2} \cdot K^{\prime-2}$, where $K^{\prime}=J K J$. Then $K$ is affiliated with $\mathscr{M}_{0}^{o}$. Since $S_{p}(\Delta)$ is an infinite set, it follows that $S_{p}(K)$ is an infinite set, which implies that $\mathscr{M}_{0}^{\sigma}$ is infinitely dimensional. By Theorem 4.8 our assertion holds.

\section{§5. Standardness of Trace Functionals on $O_{p}^{*}$-Algebras}

Gudder and Hudson [8] have studied positive linear functionals on the canonical algebra $\mathscr{A}$ for one degree of freedom which induce unbounded representations of $\mathscr{A}$ on the Hilbert space of HilbertSchmidt operators. In this section we study the standardness of trace functionals on $O_{p}^{*}$-algebras using their idea. Woronowicz [36] and Sherman [30] have dealt with trace representations of positive linear functionals on the $O_{p}^{*}$-algebra $\mathscr{L}^{+}(\mathscr{S}), \mathscr{S}$ being the Schwartz space, and the $O_{p}^{*}$-algebra generated by the position and the moment 
operators. Lassner and Timmermann [20] and Schmüdgen [28] have extended their results to more general $O_{p}^{*}$-algebras. We here investigated under what conditions trace functionals on a certain $O_{p}^{*}-$ algebra are modular (or standard).

We first prepare some lemmas. We denote by $\mathfrak{S} \otimes \overline{\mathfrak{S}}$ the Hilbert space with inner product $\langle\mid\rangle$ of Hilbert-Schmidt operators on a separable Hilbert space $\mathfrak{S}$, and by $\mathscr{D} \otimes \overline{\mathfrak{K}}$ the subspace of $\mathscr{S} \otimes \overline{\mathscr{F}}$ consisting of Hilbert-Schmidt operators whose range are contained in a subspace $\mathscr{D}$ of $\mathfrak{S}$. Let $K$ be a densely defined closed operator in $\mathfrak{S}$. We define densely defined closed operators $\pi^{\prime \prime}(K)$ and $\pi^{\prime}(K)$ as follows:

$$
\begin{aligned}
& \left\{\begin{array}{l}
\mathscr{D}\left(\pi^{\prime \prime}(K)\right)=\{T \in \mathscr{S} \otimes \overline{\mathfrak{S}} ; \quad K T \in \mathscr{S} \otimes \overline{\mathfrak{S}}\}, \\
\pi^{\prime \prime}(K) T=K T, T \in \mathscr{D}\left(\pi^{\prime \prime}(K)\right) ;
\end{array}\right. \\
& \left\{\begin{array}{l}
\mathscr{D}\left(\pi^{\prime}(K)\right)=\{T \in \mathfrak{S} \otimes \overline{\mathfrak{S}} ; \overline{T K} \in \mathfrak{S} \otimes \overline{\mathfrak{S}}\}, \\
\pi^{\prime}(K) T=\overline{T K}, T \in \mathscr{D}\left(\pi^{\prime}(K)\right) .
\end{array}\right.
\end{aligned}
$$

Lemma 5.1. (1) $\pi^{\prime \prime}(\mathscr{B}(\mathfrak{S}))$ is a von Neumann algebra on $\mathfrak{S} \otimes \overline{\mathscr{F}}$ such that $\pi^{\prime \prime}(\mathscr{B}(\mathfrak{S}))^{\prime}=\pi^{\prime}(\mathscr{B}(\mathfrak{S}))$ and $\pi^{\prime \prime}(\mathscr{B}(\mathfrak{S}))=J \pi^{\prime}(\mathscr{B}(\mathfrak{S})) J$, where $J$ denotes the isometry on $\mathscr{S} \otimes \overline{\mathscr{S}}$ defined by

$$
J T=T^{*} \text {. }
$$

Let $S$ and $K$ be positive self-adjoint operators in $\mathfrak{S}$. Then the following statements hold.

(2) $\pi^{\prime \prime}(K)$ is a positive self-adjoint operator in $\mathfrak{S} \otimes \overline{\mathscr{F}}$ affiliated with the von Neumann algebra $\pi^{\prime \prime}(\mathscr{B}(\mathfrak{E}))$.

(3) $\pi^{\prime}(K)$ is a positive self-adjoint operator in $\mathscr{S} \otimes \overline{\mathscr{S}}$ affiliated with $\pi^{\prime}(\mathscr{B}(\mathfrak{S}))$.

(4) $J \mathscr{D}\left(\pi^{\prime \prime}(K)\right)=\mathscr{D}\left(\pi^{\prime}(K)\right)$ and $\pi^{\prime \prime}(K)=J \pi^{\prime}(K) J$.

(5) $\pi^{\prime \prime}(K) \cdot \pi^{\prime}(S)=\pi^{\prime}(S) \cdot \pi^{\prime \prime}(K)$, which is a positive self-adjoint operator in $\mathfrak{S}_{\mathcal{O}} \otimes \overline{\mathfrak{S}}$, where $A \cdot B$ denotes the closure $\overline{A B}$ of closed operators $A, B$ 。

Proof. (1) We can show in the same way as in ([8] Lemma 24) that $\pi^{\prime \prime}(\mathscr{B}(\mathscr{S}))^{\prime}=\pi^{\prime}(\mathscr{B}(\mathscr{E}))$. In fact, take arbitrary $\delta \in \pi^{\prime \prime}(\mathscr{B}(\mathscr{E}))^{\prime}$ 。 For each $\xi, \eta \in \mathfrak{S}$ a sesquilinear form on $\mathscr{S} \otimes \mathfrak{S}$ defined by

$$
(x, y) \in \mathfrak{F} \times \mathfrak{S} \longrightarrow\langle\delta(x \otimes \bar{\eta}) \mid y \otimes \bar{\xi}\rangle
$$


is continuous, and hence

$$
\langle\delta(x \otimes \bar{\eta}) \mid y \otimes \bar{\xi}\rangle=(\Gamma(\xi, \eta) x \mid y)
$$

for some $\Gamma(\xi, \eta) \in \mathscr{B}(\mathscr{S})$. Since $\delta \in \pi^{\prime \prime}(\mathscr{B}(\mathscr{S}))^{\prime}$, it follows that $\Gamma(\xi, \eta)$ $\in \mathscr{B}(\mathscr{S})^{\prime}=\mathbb{C} I$, so that $\Gamma(\xi, \eta)=\lambda(\xi, \eta) I$ for some $\lambda(\xi, \eta) \in \mathbb{C}$. Further, $\lambda$ is a continuous sesquilinear form on $\mathscr{S} \times \mathfrak{S}$, and hence $\lambda(\xi, \eta)=$ $(C \xi \mid \eta)$ for some $C \in \mathscr{B}(\mathscr{C})$, which implies $\delta=\pi^{\prime}(C)$. The converse inclusion is obvious. It is clear that $\pi^{\prime \prime}(\mathscr{B}(\mathscr{S}))=J \pi^{\prime}(\mathscr{B}(\mathscr{S})) J$, so that $\pi^{\prime \prime}(\mathscr{B}(\mathscr{C}))$ is a von Neumann algebra.

(2) It is clear that $\pi^{\prime \prime}(K)$ is a positive operator in $\mathscr{S} \otimes \overline{\mathscr{S}}$ affiliated with $\pi^{\prime \prime}(\mathscr{B}(\mathscr{C}))$. Take arbitrary $A \in \mathscr{D}\left(\pi^{\prime \prime}(K)^{*}\right)$. Then we can show $\mathscr{D}(K A)=\mathfrak{S}$. By the closed graph theorem $K A$ is a bounded linear operator. Since

$$
\begin{aligned}
\sum_{k=1}^{\infty}\left\|K A e_{k}\right\|^{2} & =\sum_{k=1}^{\infty}\left\|\pi^{\prime \prime}(K) * A\left(e_{k} \otimes \overline{e_{k}}\right)\right\|_{2}^{2} \\
& =\sum_{k=1}^{\infty}\left\|\pi^{\prime \prime}(K) * A e_{k}\right\|^{2}<\infty
\end{aligned}
$$

where $\|\cdot\|_{2}$ denotes the norm generated by the inner product $\langle\mid\rangle$ on $\mathscr{S} \otimes \overline{\mathscr{S}}$ and $\left\{e_{k}\right\}$ is an orthonormal basis in $\mathfrak{S}$, we have $K A \in \mathfrak{S} \otimes \overline{\mathcal{F}_{2}}$. Hence $\pi^{\prime \prime}(K)$ is self-adjoint.

(4) This is trivial.

(3) This follows from (2) and (4).

(5) This is proved by using the theory of spectral resolutions of self-adjoint operators.

Lemma 5.2. Suppose $\Omega$ is a non-singular positive Hilbert-Schmidt operator on $\mathfrak{S C}_{\text {. Then }} \pi^{\prime \prime}(\mathscr{B}(\mathscr{S})) \Omega$ is an achieved left Hilbert algebra in $\mathfrak{S} \otimes \overline{\mathfrak{S}}$ equipped with the multiplication $\left(\pi^{\prime \prime}(A) \Omega\right)\left(\pi^{\prime \prime}(B) \Omega\right)=\pi^{\prime \prime}(A B) \Omega$ and the involution $\pi^{\prime \prime}(A) \Omega \rightarrow \pi^{\prime \prime}\left(A^{*}\right) \Omega$. Further, the modular conjugation operator $J_{\Omega}$ of $\pi^{\prime \prime}(\mathscr{B}(\mathfrak{S})) \Omega$ equals the isometry $J$ on $\mathscr{S} \otimes \overline{\mathcal{S}}$, and the modular operator $\Delta_{\Omega}$ of $\pi^{\prime \prime}(\mathscr{B}(\mathfrak{S})) \Omega$ equals the operator $\pi^{\prime}\left(\Omega^{-2}\right) \pi^{\prime \prime}\left(\Omega^{2}\right)$.

Proof. Since $\Omega$ is non-singular, it follows that $\pi^{\prime \prime}(\mathscr{B}(\mathscr{S})) \Omega$ is dense in $\mathscr{S} \otimes \overline{\mathscr{S}}$. By Lemma 5. 1 we have $J \pi^{\prime \prime}(\mathscr{B}(\mathscr{S})) J=\pi^{\prime}(\mathscr{B}(\mathscr{S}))$ and $J \Omega=\Omega$. Hence, $\pi^{\prime}(\mathscr{B}(\mathscr{S})) \Omega$ is dense in $\mathscr{S} \otimes \overline{\mathscr{F}}$, which implies that $\pi^{\prime \prime}(\mathscr{B}(\mathscr{S})) \Omega$ is an achieved left Hilbert algebra in $\mathscr{S} \otimes \overline{\mathcal{S}}$. Let $S_{\Omega}$ be the closure of the involution $\pi^{\prime \prime}(A) \Omega \rightarrow \pi^{\prime \prime}\left(A^{*}\right) \Omega$ of $\pi^{\prime \prime}(\mathscr{B}(\mathscr{S})) \Omega$. We show $J_{\Omega}=J$. The isometry $J$ on $\mathfrak{S C} \otimes \overline{\mathcal{S}}$ satisfies 
(5. 1)

$$
\left\{\begin{array}{c}
\langle J S \mid J T\rangle=\langle T \mid S\rangle \quad \text { for each } S, T \in \mathfrak{S} \otimes \overline{\mathfrak{S}} ; \\
J^{2}=I ; \\
J \pi^{\prime \prime}(A) J=\pi^{\prime}\left(A^{*}\right) \quad \text { for each } A \in \mathscr{B}(\mathfrak{S}) ; \\
J \Omega=\Omega .
\end{array}\right.
$$

For each $T \in \mathfrak{S} \otimes \overline{\mathscr{F}}$ we have

$$
J \pi^{\prime}\left(\Omega^{-1}\right) \pi^{\prime \prime}(\Omega) \pi^{\prime \prime}(T) \Omega=J \pi^{\prime \prime}(\Omega) T=S_{\Omega} \pi^{\prime \prime}(T) \Omega .
$$

Let $\left\{e_{n}\right\}_{n=1,2, \ldots}$ be the orthonormal basis in $\mathfrak{S}$ consisting of eigenvectors of non-zero eigenvalues $\left\{\lambda_{n}\right\}_{n=1,2, \ldots}$ of $\Omega$. We now put

$$
P_{n}=\sum_{k=1}^{n} e_{k} \otimes \overline{e_{k}} \quad(n=1,2, \ldots) \text {. }
$$

Since

$$
\begin{aligned}
\| \pi^{\prime \prime} & \left(P_{n} A P_{n}\right) \Omega-\pi^{\prime \prime}(A) \Omega \|_{2} \\
& \leqq\left\|\pi^{\prime \prime}\left(P_{n} A P_{n}\right) \Omega-\pi^{\prime \prime}\left(P_{n} A\right) \Omega\right\|_{2}+\left\|\pi^{\prime \prime}\left(P_{n} A\right) \Omega-\pi^{\prime \prime}(A) \Omega\right\|_{2} \\
& \leqq\|A\|\left\|P_{n} \Omega-\Omega\right\|_{2}+\left\|\Omega A^{*} P_{n}-\Omega A^{*}\right\|_{2} \\
& =\|A\|\left(\sum_{k=n+1}^{\infty}\left\|\Omega e_{k}\right\|^{2}\right)^{\frac{1}{2}}+\left(\sum_{k=n+1}^{\infty}\left\|\Omega A^{*} e_{k}\right\|^{2}\right)^{\frac{1}{2}}
\end{aligned}
$$

for each $A \in \mathscr{B}(\mathscr{S})$, we have

$$
\lim _{n \rightarrow \infty} \pi^{\prime \prime}\left(P_{n} A P_{n}\right) \Omega=\pi^{\prime \prime}(A) \Omega
$$

for each $A \in \mathscr{B}(\mathscr{S})$. Since $P_{n} A P_{n} \in \mathscr{S} \otimes \overline{\mathscr{E}}$ for each $A \in \mathscr{B}(\mathscr{S})$, it follows that $\pi^{\prime \prime}(\mathscr{S} \otimes \overline{\mathscr{S}}) \Omega$ is dense in the Hilbert space $\mathscr{D}\left(S_{\Omega}\right)$. Hence, we have by (5.2)

$$
S_{\Omega} \subset J \pi^{\prime}\left(\Omega^{-1}\right) \pi^{\prime \prime}(\Omega) \text {. }
$$

Since $\pi^{\prime}\left(\Omega^{-1}\right) \pi^{\prime \prime}(\Omega)$ is a positive self-adjoint operator in $\mathfrak{S} \otimes \overline{\mathfrak{K}}$ by Lemma 5. 1, we have

$$
\begin{aligned}
\left\langle\Omega \mid \pi^{\prime \prime}(A) J \pi^{\prime \prime}(A) J \Omega\right\rangle & =\left\langle S_{\Omega} \pi^{\prime \prime}(A) \Omega \mid J \pi^{\prime \prime}(A) \Omega\right\rangle \quad \text { (by 5.4) } \\
& =\left\langle J \pi^{\prime}\left(\Omega^{-1}\right) \pi^{\prime \prime}(\Omega) \pi^{\prime \prime}(A) \Omega \mid J \pi^{\prime \prime}(A) \Omega\right\rangle \\
& =\left\langle\pi^{\prime \prime}(A) \Omega \mid \pi^{\prime}\left(\Omega^{-1}\right) \pi^{\prime \prime}(\Omega) \pi^{\prime \prime}(A) \Omega\right\rangle \\
& \geqq 0
\end{aligned}
$$

for each $A \in \mathscr{B}(\mathfrak{S})$. It hence follows from ([1] Theorem 1) that $J=J_{\Omega}$. By (5.4) we have $\Delta_{\Omega}^{\frac{1}{2}} \subset \pi^{\prime}\left(\Omega^{-1}\right) \pi^{\prime \prime}(\Omega)$. By the maximality of self-adjoint operators, we have $\Delta_{\Omega}^{\frac{1}{2}}=\pi^{\prime}\left(\Omega^{-1}\right) \pi^{\prime \prime}(\Omega)$. This completes the proof.

Lemma 5.3. Let $\mathscr{D}$ be a dense subspace of $\mathfrak{S}$ such that $\mathscr{L}^{+}(\mathscr{D})$ 
contains the inverse of a Hilbert-Schmidt operator. Then the following statements hold.

(1) Put

$$
\pi(X) T=X T
$$

for $X \in \mathscr{L}^{+}(\mathscr{D})$ and $T \in \mathscr{D} \otimes \overline{\mathcal{H}}$. Then $\pi$ is a*-representation of $\mathscr{L}^{+}(\mathscr{D})$ on $\mathfrak{S} \otimes \overline{\mathfrak{S}}$ with domain $\mathscr{D} \otimes \overline{\mathfrak{S}}$.

(2) Suppose $\mathscr{M}$ is a closed (resp. self-adjoint) $O_{p}^{*}$-algebra on $\mathscr{D}$ such that $\mathscr{M}^{\prime}=\boldsymbol{C I}$. Then $\pi(\mathscr{M})$ is a closed (resp. self-adjoint) $O_{p}^{*}$-algebra on $\mathscr{D} \otimes \overline{\mathscr{S}}$ satisfying $\pi(\mathscr{M})^{\prime}=\pi^{\prime}(\mathscr{B}(\mathfrak{E}))$ and $\pi(\mathscr{M})^{\prime \prime}=\pi^{\prime \prime}(\mathscr{B}(\mathfrak{S}))$.

Proof. This is similar to the proofs of ([8] Lemmas 22, 23, 24).

Theorem 5.4. Suppose $(\mathscr{M}, \mathscr{D}, \Omega)$ is a system satisfying the conditions;

(i) $\mathscr{M}$ is a closed $O_{p}^{*}$-algebra on $\mathscr{D}$ with identity I such that $\mathscr{M}^{\prime}=$ CI;

(ii) there exists an element $T$ of $\mathscr{L}^{+}(\mathscr{D})$ such that $\bar{T}^{-1} \in \mathfrak{S} \otimes \overline{\mathscr{S}}$;

(iii) $\Omega$ is a positive operator contained in $\mathscr{D} \otimes \overline{\mathcal{S}}$ satisfying

(iii) ${ }_{1} \overline{\pi(\mathscr{M}) \Omega^{t} \pi(\mathscr{M})}=\mathscr{D} \otimes \overline{\mathcal{S}}$;

(iii) ${ }_{2} \Omega^{i t} \mathscr{D}=\mathscr{D}$ for all $t \in \boldsymbol{R}$.

Then the following statements hold.

(1) $\pi(\mathscr{M}) \Omega$ is a modular unbounded left Hilbert algebra in $\mathfrak{S} \otimes \overline{\mathcal{S}}$ with $\mathscr{D}_{\pi(\mathscr{M}) \Omega}=\mathscr{D} \otimes \overline{\mathcal{S}} \quad$ satisfying $\quad(\pi(\mathscr{M}) \Omega)^{\prime \prime}=\pi^{\prime \prime}(\mathscr{B}(\mathfrak{S})) \Omega, \quad J_{\pi(\mathscr{M}) \Omega}^{\prime \prime}=J_{\Omega}=J$, $\Delta_{\pi(\mathscr{M}) \Omega}^{\prime \prime}=\Delta_{\Omega}=\pi^{\prime}\left(\Omega^{-2}\right) \pi^{\prime \prime}\left(\Omega^{2}\right)$ and $\mathscr{U}(\pi(\mathscr{M}) \Omega)=\pi\left(\mathscr{L}^{+}(\mathscr{D})\right)$. In particular, $\pi\left(\mathscr{L}^{+}(\mathscr{D})\right) \Omega$ is a standard unbounded left Hilbert algebra in $\mathfrak{S} \otimes \overline{\mathfrak{S}}$.

(2) Put

$$
\sigma_{t}^{\Omega}(X)=\Omega^{2 i t} X \Omega^{-2 i t}, \quad X \in \mathscr{L}^{+}(\mathscr{D}), \quad t \in \boldsymbol{R}_{.}
$$

Then $\left\{\sigma_{t}^{Q}\right\}_{t \in \boldsymbol{R}}$ is a one-parameter group of automorphisms of $\mathscr{L}^{+}(\mathscr{D})$ satisfying $\pi\left(\sigma_{t}^{\Omega}(X)\right)=\Delta_{\Omega}^{i t} \pi(X) \Delta_{\Omega}^{-i t}$ for all $X \in \mathscr{L}^{+}(\mathscr{D})$ and $t \in \boldsymbol{R}_{\mathbf{b}}$

(3) Put

$$
\phi_{\Omega}(X)=\operatorname{Tr} X \Omega^{2}, \quad X \in \mathscr{L}^{+}(\mathscr{D}) .
$$

Then the trace functional $\phi_{\Omega}$ on $\mathscr{M}$ is modular and $\left(\mathscr{L}^{+}(\mathscr{D}),\left\{\sigma_{t}^{\Omega}\right\}, \phi_{\Omega}\right)$ is a standard system.

(4) Suppose $\mathscr{L}^{+}(\mathscr{D})$ is self-adjoint. Then every strongly positive linear functional $\phi$ on $\mathscr{L}^{+}(\mathscr{D})$ which satisfies the $K M S$-condition with respect to $\left\{\sigma_{t}^{\Omega}\right\}$ is represented as $\phi=\gamma \phi_{\Omega}$ for some constant $\gamma>0$. 
Proof. (1) It follows from (iii) ${ }_{1}$ and Lemma 5.3 that $\pi^{\prime \prime}(\mathscr{B}(\mathfrak{S})) \Omega$ is dense in $\mathfrak{S} \otimes \overline{\mathfrak{K}}$, which implies that $\Omega$ is non-singular. By Lemmas 5. 2, $5.3 \pi(\mathscr{M}) \Omega$ is an unbounded left Hilbert algebra in $\mathfrak{S} \otimes \overline{\mathcal{S}}$ such that $\mathscr{D}\left(\hat{\pi}_{\pi(\mathscr{M}) \Omega}\right)=\mathscr{D} \otimes \overline{\mathscr{S}},(\pi(\mathscr{M}) \Omega)^{\prime \prime}=\pi^{\prime \prime}(\mathscr{B}(\mathfrak{E})) \Omega$ and $S_{\pi(\mathscr{M}) \Omega}^{\prime \prime}=J \pi^{\prime}\left(\Omega^{-1}\right)$ $\pi^{\prime \prime}(\Omega)$. By (iii) 2 we have

$$
\begin{aligned}
\Delta_{\pi(\mathcal{M}) \Omega}^{\prime \prime i t} \mathscr{D} \otimes \overline{\mathscr{S}} & =\pi^{\prime}\left(\Omega^{-2 i t}\right) \pi^{\prime \prime}\left(\Omega^{2 i t}\right) \mathscr{D} \otimes \overline{\mathfrak{S}_{\mathfrak{S}}} \\
& =\mathscr{D} \otimes \overline{\mathfrak{S}_{\mathfrak{C}}}
\end{aligned}
$$

for all $t \in \mathbb{R}$. Thus $\pi(\mathscr{M}) \Omega$ is a modular unbounded left Hilbert algebra in $\mathscr{S} \otimes \overline{\mathfrak{S}}$ with $\mathscr{D}_{\pi(\mathscr{M}) \Omega}=\mathscr{D} \otimes \overline{\mathfrak{S}_{\text {. }}}$. We shall show $\mathscr{U}(\pi(\mathscr{M}) \Omega)$ $=\pi\left(\mathscr{L}^{+}(\mathscr{D})\right)$. Since $\mathscr{U}(\pi(\mathscr{M}) \Omega)=\left\{\delta \in \mathscr{L}^{+}(\mathscr{D} \otimes \overline{\mathfrak{S}}) ; \bar{\delta}\right.$ is affiliated with $\left.\pi^{\prime \prime}(\mathscr{B}(\mathscr{C}))\right\}$, we have $\pi\left(\mathscr{L}^{+}(\mathscr{D})\right) \subset \mathscr{U}(\pi(\mathscr{M}) \Omega)$. Take arbitrary $\delta \in$ $\mathscr{U}(\pi(\mathscr{M}) \Omega)$. Then there exists a sequence $\left\{A_{n}\right\}$ in $\mathscr{B}(\mathfrak{E})$ such that $\lim _{n \rightarrow \infty} \pi^{\prime \prime}\left(A_{n}\right) T=\delta T$ and $\lim _{n \rightarrow \infty} \pi^{\prime \prime}\left(A_{n}^{*}\right) T=\delta^{+} T$ for all $T \in \mathscr{D} \otimes \overline{\mathcal{S}}$, which implies that $\lim _{n \rightarrow \infty} A_{n} \xi$ and $\lim _{n \rightarrow \infty} A_{n}^{*} \xi$ exist for each $\xi \in \mathscr{D}$. We now put

$$
X \xi=\lim _{n \rightarrow \infty} A_{n} \xi \text { and } Y \xi=\lim _{n \rightarrow \infty} A_{n}^{*} \xi
$$

for each $\xi \in \mathscr{D}$. For each $k \in \mathbb{N}$ and $\xi \in \mathscr{D}$ we have

$$
\begin{aligned}
\| X \xi-\delta\left(\xi \otimes \overline{e_{k}}\right) e_{k} & \left\|^{2}+\sum_{\substack{i=1 \\
i \neq k}}^{\infty}\right\| \delta\left(\xi \otimes \overline{e_{k}}\right) e_{i} \|^{2} \\
& =\lim _{n \rightarrow \infty} \sum_{i=1}^{\infty}\left\|A_{n}\left(\xi \otimes \overline{e_{k}}\right) e_{i}-\delta\left(\xi \otimes \overline{e_{k}}\right) e_{i}\right\|^{2} \\
& =\lim _{n \rightarrow \infty}\left\|\pi^{\prime \prime}\left(A_{n}\right)\left(\xi \otimes \overline{e_{k}}\right)-\delta\left(\xi \otimes \overline{e_{k}}\right)\right\|_{2}^{2} \\
& =0,
\end{aligned}
$$

where $\left\{e_{k}\right\}$ is an orthonormal basis in $\mathfrak{S}_{\text {. }}$ Hence we have

$$
\delta\left(\xi \otimes \overline{e_{k}}\right) e_{i}= \begin{cases}X \xi, & k=i \\ 0, & k \neq i\end{cases}
$$

for each $\xi \in \mathscr{D}$. Similarly we have

$$
\delta^{+}\left(\xi \otimes \bar{e}_{k}\right) e_{i}= \begin{cases}Y \xi, & k=i \\ 0, & k \neq i\end{cases}
$$

for each $\xi \in \mathscr{D}$. By (5.5), (5.6) we have $X, Y \in \mathscr{L}(\mathscr{D})$. Since $(X \xi \mid \eta)=(\xi \mid Y \eta)$ for each $\xi, \eta \in \mathscr{D}$, it follows that $X \in \mathscr{L}^{+}(\mathscr{D})$ and $X^{+}=Y$. For each $T \in \mathscr{D} \otimes \overline{\mathscr{S}}$ we have

$$
\lim _{n \rightarrow \infty}|| \sum_{k=1}^{n} T e_{k} \otimes \overline{e_{k}}-\left.T\right|_{12} ^{\prime}=0
$$




$$
\begin{array}{rlrl}
\lim _{n \rightarrow \infty}\left\|\delta\left(\sum_{k=n}^{m} T e_{k} \otimes \overline{e_{k}}\right)\right\|_{2}^{2} & & (m>n) \\
& =\lim _{n \rightarrow \infty} \sum_{k=n}^{m}\left\|X T e_{k}\right\|^{2} & & (\text { by } 5.5) \\
& =0, &
\end{array}
$$

and hence

$$
\begin{aligned}
\delta T & =\lim _{n \rightarrow \infty} \delta\left(\sum_{k=1}^{n} T e_{k} \otimes \overline{e_{k}}\right) \\
& =\lim _{n \rightarrow \infty} \pi(X)\left(\sum_{k=1}^{n} T e_{k} \otimes \overline{e_{k}}\right) \\
& =\pi(X) T .
\end{aligned}
$$

Hence, $\delta=\pi(X) \in \pi\left(\mathscr{L}^{+}(\mathscr{D})\right)$.

(2) This follows from (1).

(3) This follows from (1), (2) and Theorem 3.9.

(4) Since $\mathscr{L}^{+}(\mathscr{D})$ is self-adjoint and $\phi$ is strongly positive, it follows from ([36] Theorem 3) and ([20] Lemma 2.4) that

$$
\phi(X)=\operatorname{Tr} X v^{2}, \quad X \in \mathscr{L}^{+}(\mathscr{D})
$$

for some positive operator $v$ in $\mathscr{D} \otimes \overline{\mathscr{S}}$. We now put

$$
\phi=\phi+\phi_{\Omega} \text {. }
$$

Since $\phi$ is strongly positive, it is represented as

$$
\phi(X)=\operatorname{Tr} X \rho^{2}=\langle\pi(X) \rho \mid \rho\rangle, \quad X \in \mathscr{L}^{+}(\mathscr{D})
$$

for some positive operator $\rho$ in $\mathscr{D} \otimes \overline{\mathscr{C}}$. Then $\rho=\left(v^{2}+\Omega^{2}\right)^{\frac{1}{2}}$, and so $\rho$ is non-singular, which implies

$$
\overline{\pi\left(\mathscr{L}^{+}(\mathscr{D})\right) \rho^{t}}{ }_{\pi\left(\mathscr{L}^{+}(\mathscr{D})\right)}=\mathscr{D} \otimes \overline{\mathscr{S}} .
$$

In fact, take arbitrary $T \in \mathscr{D} \otimes \overline{\mathcal{F}}$. Let $\left\{e_{n}\right\}$ be an orthonormal basis in $\mathfrak{S}$ consisting of eigenvectors of non-zero eigenvalues $\left\{\lambda_{n}\right\}$ of $\rho$, and let $A_{n}=\sum_{k=1}^{n} \frac{1}{\lambda_{k}} e_{k} \otimes \overline{e_{k}}$. Then we have $T A_{n} \in \mathscr{L}^{+}(\mathscr{D})$ for $n \in N$ and

$$
\begin{aligned}
\lim _{n \rightarrow \infty}\left\|\pi(X) \pi\left(T A_{n}\right) \rho-\pi(X) T\right\|_{2}^{2} & =\lim _{n \rightarrow \infty} \sum_{k=1}^{\infty}\left\|X T A_{n} \rho e_{k}-X T e_{k}\right\|^{2} \\
& =\lim _{n \rightarrow \infty} \sum_{k=n+1}^{\infty}\left\|X T e_{k}\right\|^{2} \\
& =0
\end{aligned}
$$

for all $X \in \mathscr{L}^{+}(\mathscr{D})$. Hence, $T \in \overline{\pi\left(\mathscr{L}^{+}(\mathscr{D})\right) \rho^{t}} \pi_{\left(\mathscr{L}^{+}(\mathscr{D})\right)}$. By Lemmas 5. 2, $5.3 \pi\left(\mathscr{L}^{+}(\mathscr{D})\right) \rho$ is an unbounded left Hilbert algebra in $\mathscr{S} \otimes \overrightarrow{\mathscr{S}}$ with 
$S_{\pi\left(\mathscr{L}^{+}(\mathscr{D})\right) \rho}^{\prime \prime}=S_{\rho}=J \pi^{\prime}\left(\rho^{-1}\right) \pi^{\prime \prime}(\rho)$. It follows from (5.7) and Lemma 5.3 that $\pi_{\psi}$ is self-adjoint and $\left(\mathscr{L}^{+}(\mathscr{D}),\left\{\sigma_{t}^{O}\right\}, \phi\right)$ is a KMS-system. By Lemma 3.8 we have

$$
\pi\left(\sigma_{t}^{Q}(X)\right) \rho=\Delta_{\rho}^{i t} \pi(X) \rho
$$

for all $X \in \mathscr{L}^{+}(\mathscr{D})$ and $t \in \mathbb{R}$, which implies

$$
\begin{aligned}
\left\langle\pi(X) \pi(Y) \rho \mid \Delta_{\rho}^{i t} T\right\rangle & =\left\langle\Delta_{\rho}^{-i t} \pi(X Y) \rho \mid T\right\rangle \\
& =\left\langle\pi\left(\sigma_{-t}^{Q}(X Y)\right) \rho \mid T\right\rangle \\
& =\left\langle\pi(Y) \rho \mid \Delta_{\rho}^{i t} \pi\left(\sigma_{-t}^{Q}\left(X^{+}\right)\right) T\right\rangle
\end{aligned}
$$

for each $X, Y \in \mathscr{L}^{+}(\mathscr{D}), T \in \mathscr{D} \otimes \overline{\mathscr{S}}$ and $t \in \mathbb{R}_{R}$. It hence follows from the self-adjointness of $\pi\left(\mathscr{L}^{+}(\mathscr{D})\right)$ that

$$
\Delta_{\rho}^{i t} \mathscr{D} \otimes \overline{\mathscr{S}} \subset \mathscr{D} \otimes \overline{\mathscr{C}} \text { and } \Delta_{\rho}^{i t} \pi(X) \Delta_{\rho}^{-i t}=\pi\left(\sigma_{t}^{\mathscr{P}}(X)\right)
$$

for all $X \in \mathscr{L}^{+}(\mathscr{D})$ and $t \in \mathbb{R}$, which implies that $\left(\mathscr{L}^{+}(\mathscr{D}),\left\{\sigma_{t}^{\mathscr{Q}}\right\}, \phi\right)$ is a standard system. By (5.8) we have

$$
\begin{aligned}
\pi\left(\rho^{2 i t} X \rho^{-2 i t}\right)=\Delta_{\rho}^{i t} \pi(X) \Delta_{\rho}^{-i t} & =\pi\left(\sigma_{t}^{Q}(X)\right) \\
& =\pi\left(\Omega^{2 i t} X \Omega^{-2 i t}\right)
\end{aligned}
$$

for all $X \in \mathscr{L}^{+}(\mathscr{D})$ and $t \in \mathbb{R}$, and hence $\rho^{2 i t} X \rho^{-2 i t}=\Omega^{2 i t} X \Omega^{-2 i t}$ for all $X \in \mathscr{L}^{+}(\mathscr{D})$ and $t \in \mathbb{R}$. Hence, $\rho^{2 i t} \mathscr{Q}^{-2 i t} \in \mathscr{L}^{+}(\mathscr{D})^{\prime}=\mathbb{C} I$ for all $t \in \mathbb{R}$, which implies $\rho=\gamma \Omega$ for some constant $\gamma>0$. Hence we have

$$
\phi(X)=\phi(X)-\phi_{\Omega}(X)=\left(\gamma^{2}-1\right) \phi_{\Omega}(X)
$$

for all $X \in \mathscr{L}^{+}(\mathscr{D})$. This completes the proof.

We give some examples of $(\mathscr{M}, \mathscr{D}, \Omega$ ) satisfying the conditions (i), (ii) and (iii) of Theorem 5.4. Let $\mathscr{S}=\mathscr{S}(\mathbb{R})$ be the Schwartz space of infinitely differentiable rapidly decreasing functions and $\left\{f_{n}\right\}_{n=0,1,2, \ldots}$ be the normalized Hermite functions; that is,

$$
f_{n}(t)=\left(2^{n} n !\right)(-1)^{n} \pi^{-\frac{1}{t}} e^{\frac{1}{t} t^{2}}\left(\frac{d}{d t}\right)^{n} e^{-t^{2}} .
$$

Then $\left\{f_{n}\right\}$ forms an orthonormal basis in the Hilbert space $L^{2}=L^{2}(\mathbb{R})$

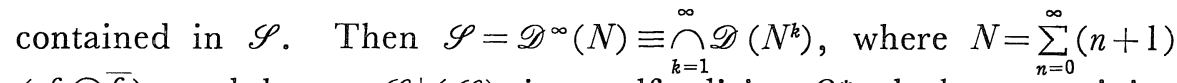
$\left(f_{n} \otimes \overline{f_{n}}\right)$, and hence $\mathscr{L}^{+}(\mathscr{S})$ is a self-adjoint $O_{p}^{*}$-algebra containing the inverse $N$ of a positive Hilbert-Schmidt operator. We put

$$
\begin{aligned}
& \boldsymbol{s}=\left\{\left\{\alpha_{n}\right\} \subset \mathbb{C} ; \sup _{n} n^{k}\left|\alpha_{n}\right|<\infty \quad \text { for each } k \in \mathbb{N}\right\}, \\
& \boldsymbol{s}_{+}=\left\{\left\{\alpha_{n}\right\} \in \boldsymbol{s} ; \alpha_{n}>0 \text { for } n=0,1,2, \ldots\right\},
\end{aligned}
$$




$$
\Omega_{\left\{\alpha_{n}\right\}}=\sum_{n=0}^{\infty}\left|\alpha_{n}\right| f_{n} \otimes \overline{f_{n},} \quad\left\{\alpha_{n}\right\} \in \boldsymbol{s} .
$$

Then it is easily shown that $\Omega_{\left\{\alpha_{n}\right\}} \in \mathscr{D}^{\infty}\left(\pi^{\prime \prime}(N)\right)=\mathscr{S} \otimes \overline{L^{2}}$ and $N^{k} \Omega_{\left\{\alpha_{n}\right\}} \supset$ $\Omega_{\left\{\alpha_{n}\right\}} N^{k}$ for each $k \in \mathbb{N}$ and $\left\{\alpha_{n}\right\} \in \boldsymbol{s}_{+}$. Hence $\left(\mathscr{S}, \Omega_{\left(\alpha_{n}\right)}\right)$ satisfies the conditions (ii) and (iii) ${ }_{2}$ of Theorem 5.4, and so we have the following

Corollary 5.5. (1) Let $\mathscr{M}$ be a closed $O_{p}^{*}$-algebra on $\mathscr{S}$ with identity I such that $\mathscr{M}^{\prime}=\mathbb{C} I$, and $\left\{\alpha_{n}\right\} \in \boldsymbol{s}_{+}$. Suppose $\overline{\pi(\mathscr{M}) \Omega_{\left(\alpha_{n}\right\}}}{ }^{t}{ }_{(\mathscr{M})}=$ $\mathscr{S} \otimes \overline{L^{2}}$. Then $\pi(\mathscr{M}) \Omega_{\left\{\alpha_{n}\right\}}$ is a modular unbounded left Hilbert algebra in $L^{2} \otimes \bar{L}^{2}$ with $\mathscr{D}_{\pi(\mathscr{H}) \Omega_{\left\{\alpha_{n}\right\}}}=\mathscr{S} \otimes \overline{L^{2}}$ and $\mathscr{U}\left(\pi(\mathscr{M}) \Omega_{\left\{\alpha_{n}\right\}}\right)=\pi\left(\mathscr{L}^{+}(\mathscr{S})\right)$. In particular, for every $\left\{\alpha_{n}\right\} \in \boldsymbol{s}_{+} \pi\left(\mathscr{L}^{+}(\mathscr{S})\right) \Omega_{\left\{\alpha_{n}\right\}}$ is a standard unbounded left Hilbert algebra in $L^{2} \otimes \bar{L}^{2}$ with $\mathscr{D}_{\pi\left(\mathscr{L}^{+}(\mathscr{S})\right) \Omega_{\left\{\alpha_{n}\right\}}}=\mathscr{S} \otimes \overline{L^{2}}$ and

$$
\mathscr{U}\left(\pi\left(\mathscr{L}^{+}(\mathscr{S})\right) \Omega_{\left\{\alpha_{n}\right\}}\right)=\pi\left(\mathscr{L}^{+}(\mathscr{S})\right) .
$$

(2) A strongly positive linear functional $\phi$ on $\mathscr{L}^{+}(\mathscr{S})$ which satisfies the KMS-condition with respect to $\left\{\sigma_{t}^{\left.\Omega^{\left[\alpha_{n}\right.}\right\}}\right.$ for $\left\{\alpha_{n}\right\} \in \boldsymbol{s}_{+}$is represented as $\phi=\gamma \phi_{\Omega_{\left\{\alpha_{n}\right\}}}$ for some constant $\gamma>0$.

Let $\mathscr{A}$ be the canonical algebra for one degree of freedom; that is, the *-algebra generated by two self-adjoint elements $p$ and $q$ satisfying the well-known Heisenberg commutation relation: $p q-q p$ $=-i$. The Schrödinger representation $\pi_{0}$ of $\mathscr{A}$ on $L^{2}$ with domain $\mathscr{S}$ is defined by

$$
\left(\pi_{0}(p) f\right)(t)=-i\left(\frac{d f}{d t}\right)(t), \quad\left(\pi_{0}(q) f\right)(t)=t f(t) .
$$

Then $\pi_{0}$ is a faithful self-adjoint representation of $\mathscr{A}$ with a strongly cyclic vector $f_{0}(t)=\pi^{-\frac{1}{4}} e^{-\frac{t^{2}}{2}}$ satisfying $\pi_{0}(\mathscr{A})^{\prime}=C I$ [23]. We note by the following corollary that positive linear functionals on $\mathscr{A}$ given by Gudder and Hudson [8] are standard.

Corollary 5.6. Suppose $\mathscr{M}$ is an $O_{p}^{*}$-algebra on $\mathscr{S}$ containing $\pi_{0}(\mathscr{A})$ and $\left\{\alpha_{n}\right\} \in \boldsymbol{s}_{+}$satisfies

$$
0<\alpha_{n} \leqq \gamma e^{-n \beta}, \quad n \in \mathbb{N}
$$


for some $\beta>0$ and $\gamma>0$. Then $\pi(\mathscr{M}) \Omega_{\left\{\alpha_{n}\right]}$ is a standard unbounded left Hilbert algebra in $L^{2} \otimes \overline{L^{2}}$ with $\mathscr{D}_{\pi(\mathcal{M}) \Omega_{\left\{\alpha_{n}\right\}}}=\mathscr{S} \otimes \overline{L^{2}}$ and $\mathscr{U}\left(\pi(\mathscr{M}) \Omega_{\left(\alpha_{n}\right)}\right)=$ $\pi\left(\mathscr{L}^{+}(\mathscr{S})\right)$.

Proof. By ([8] Lemma 27) we have

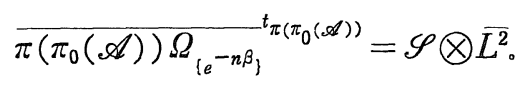

Since $\mathscr{S} \otimes \overline{L^{2}}=\mathscr{D}^{\infty}\left(\pi^{\prime \prime}(N)\right)$, it follows from Lemma 4.3 that the induced topology $t_{\mathscr{L}^{+}\left(\mathscr{S} \otimes \bar{L}^{2}\right)}$ on $\mathscr{S} \otimes \overline{L^{2}}$ is generated by a family of seminorms: $\|T\|_{n}=\left\|\pi^{\prime \prime}\left(N^{n}\right) T\right\|_{2}$ for $n \in \mathbb{N}$, and further, since $N=\overline{\pi_{0}\left(a a^{+}\right)}$, where $a=\frac{1}{\sqrt{2}}(q+i p)$ and $a^{+}=\frac{1}{\sqrt{2}}(q-i p)$, we have

$$
t_{\pi\left(\pi_{0}(\mathscr{A})\right)}=t_{\pi(\mathscr{A})}=t_{\mathscr{L}^{+}\left(\mathscr{S} \otimes \bar{L}^{2}\right)} .
$$

By (5.9) and (5.10) we have

$$
\overline{\pi(\mathscr{M}) \Omega_{\left(e^{-n \beta_{3}}\right.} \pi_{\pi(\mathscr{M})}}=\mathscr{S} \otimes \overline{L^{2}}
$$

It hence follows from Corollary 5.5 that $\pi(\mathscr{M}) \Omega_{{ }^{\left.e^{-n \beta}\right\}}}$ is a modular unbounded left Hilbert algebra in $L^{2} \otimes \bar{L}^{2}$ with $\mathscr{D}_{\pi(\mathscr{M}) \Omega}{ }_{\left(e^{-n \beta_{1}}\right.}=\mathscr{S} \otimes \bar{L}^{2}$ and $\mathscr{U}\left(\pi(\mathscr{M}) \Omega_{\left(e^{-n \beta_{1}}\right.}\right)=\pi\left(\mathscr{L}^{+}(\mathscr{S})\right)$. By ([8] Lemma 28) and Lemma $5.2 \pi\left(\pi_{0}(\mathscr{A})\right) \Omega_{t e^{-n \beta_{\}}}}$is standard, which implies that $\pi(\mathscr{M}) \Omega_{i e^{-n \beta_{\}}}}$is standard. It follows from (5.11) that for each $X \in \mathscr{L}^{+}(\mathscr{S})$ there is a net $\left\{X_{\alpha}\right\}$ in $\mathscr{M}$ such that $\lim _{\alpha} \pi(Y) \pi\left(X_{\alpha}\right) \Omega_{{ }_{i e^{-n \beta_{\}}}}}=\pi(Y) \pi(X) \Omega_{i e^{-n \beta_{\}}}}$ for each $Y \in \mathscr{M}$. Since $\pi^{\prime}(K)$ is bounded, where $K=\sum_{n=0}^{\infty} \frac{\alpha_{n}}{e^{-n \beta}}\left(f_{n} \otimes \overline{f_{n}}\right)$, we have

$$
\begin{aligned}
\lim _{\alpha} \pi(Y) \pi\left(X_{\alpha}\right) \Omega_{\left\{\alpha_{n}\right\}} & =\lim _{\alpha} \pi(Y) \pi\left(X_{\alpha}\right) \pi^{\prime}(K) \Omega_{\left\{e^{-n \beta\}}\right.} \\
& =\pi^{\prime}(K) \lim _{\alpha} \pi(Y) \pi\left(X_{\alpha}\right) \Omega_{\left\{e^{-n \beta_{3}}\right.} \\
& =\pi^{\prime}(K) \pi(Y) \pi(X) \Omega_{\left\{e^{-n \beta_{\}}}\right.} \\
& =\pi(Y) \pi(X) \Omega_{\left\{\alpha_{n}\right\}}
\end{aligned}
$$

for each $Y \in \mathscr{M}$, and hence $\pi\left(\mathscr{L}^{+}(\mathscr{S})\right) \Omega_{\left\{\alpha_{n}\right\}} \subset \overline{\subset(\mathscr{M}) \Omega_{\left\{\alpha_{n}{ }^{\prime}\right.}}{ }_{\pi(\mathscr{M})}$. Since

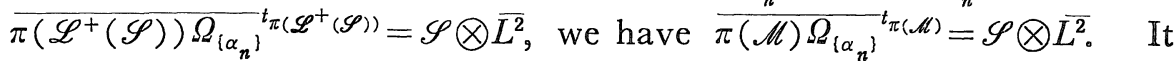


hence follows from Corollary 5.5 that $\pi(\mathscr{M}) \Omega_{\left\{\alpha_{n}\right\}}$ is a modular unbounded left Hilbert algebra in $L^{2} \otimes \overline{L^{2}}$ with $\mathscr{D}_{\pi(\mathscr{M}) \Omega_{\left\{\alpha_{n}\right\}}}=\mathscr{S} \otimes \overline{L^{2}}$ and $\mathscr{U}\left(\pi(\mathscr{M}) \Omega_{\left\{\alpha_{n}\right\}}\right)=\pi\left(\mathscr{L}^{+}(\mathscr{S})\right)$. Similarly it is shown that $\pi^{\prime \prime}\left(\mathscr{B}\left(L^{2}\right)\right) \Omega_{\left\{\alpha_{n}\right\}}$ $\subset \mathscr{D}\left(S_{\pi(\mathscr{M}) \Omega_{\left\{\alpha_{n}\right\}}}\right)$, which implies that $\pi(\mathscr{M}) \Omega_{\left\{\alpha_{n}\right\}}$ is standard. This completes the proof.

\section{§6. Structure of Some Unbounded Left Hilbert Algebra}

In this section we investigate unbounded left Hilbert algebras $\mathfrak{A}$ such that $\pi(\mathfrak{U})^{\prime \prime}$ are semifinite von Neumann algebras. We first introduce the notion of unbounded left Hilbert algebras with $E W^{*}$ extendable left regular representation.

Definition 6.1. Let $\mathfrak{A}$ be an unbounded left Hilbert algebra in $\mathfrak{S}$. If there exists a subspace $\mathscr{E}$ of $\mathscr{D}\left(\hat{\pi}_{\mathfrak{U}}\right)$ such that $\mathfrak{X} \subset \mathscr{E}, \hat{\pi}_{\mathfrak{U}}(\mathfrak{X}) \mathscr{E} \subset \mathscr{E}$ and $\pi_{\mathfrak{r}}(\mathfrak{U})^{\prime \prime} \mathscr{E}=\mathscr{E}$, then $\mathfrak{X}$ is said to be an unbounded left Hilbert algebra with $E W^{*}$-extendable left regular representation.

Let $\mathfrak{U}$ be an unbounded left Hilbert algebra in $\mathfrak{S}$ with $E W^{*}$ extendable left regular representation and $\pi$ be the left regular representation of $\mathfrak{A}$. We denote by $\mathscr{E}_{\mathfrak{r}}$ the subspace of $\mathscr{D}(\hat{\pi})$ generated by $\cup_{\mathscr{E} \in \mathscr{F}} \mathscr{E}$, where $\mathscr{F}$ is the set of all subspaces $\mathscr{E}$ of $\mathscr{D}(\hat{\pi})$ such that $\mathfrak{U} \subset \mathscr{E}, \hat{\pi}(\mathfrak{U}) \mathscr{E} \subset \mathscr{E}$, and $\pi(\mathfrak{U})^{\prime \prime} \mathscr{E}=\mathscr{E}$. Then $\mathscr{E}_{\mathscr{U}}$ is the largest element of $\mathscr{F}$. We denote by $\mathscr{U}_{m}^{*}(\mathfrak{U})$ the $O_{p}^{*}$-algebra on $\mathscr{E}_{\mathfrak{U}}$ generated by $\hat{\pi}(\mathfrak{A}) / \mathscr{E}_{\mathfrak{U}}$ and $\pi(\mathfrak{H})^{\prime \prime} / \mathscr{E}_{\mathfrak{U}}$, and denote by $\mathscr{U}_{M}^{*}(\mathfrak{U})$ the closure of $\mathscr{U}_{m}^{*}(\mathfrak{U})$ in $\left(\mathscr{L}^{+}\left(\mathscr{E}_{\mathfrak{Q}}\right), t_{w}\right)$.

Lemma 6.2. Suppose $\mathfrak{A}$ is an unbounded left Hilbert algebra in $\mathfrak{S}$ with $E W^{*}$-extendable left regular representation. Then the following statements hold.

(1) $\hat{\pi}$ is standard.

(2) $\mathscr{U}_{m}^{*}(\mathfrak{U})$ is a closed EW*-algebra on $\mathscr{E}_{\mathfrak{U}}$ over $\pi(\mathfrak{U})^{\prime \prime}$ which is minimum among $E W^{*}$-algebras on $\mathscr{E}_{\mathfrak{x}}$ over $\pi(\mathfrak{H})^{\prime \prime}$ containing $\hat{\pi}(\mathfrak{H}) / \mathscr{E}_{\mathfrak{U}}$, which is called the minimum left $E W^{*}$-algebra of $\mathfrak{A}$.

(3) $\mathscr{U}_{M}^{*}(\mathfrak{U})$ is maximum among $E W^{*}$-algebras on $\mathscr{E}_{\mathfrak{U}}$ over $\pi(\mathfrak{X})^{\prime \prime}$ containing $\hat{\pi}(\mathfrak{U}) / \mathscr{E}_{\mathfrak{2}}$, which is said to be the maximum left $E W^{*}$-algebra 
of $\mathfrak{N}$, which equals $\left(\hat{\pi}(\mathfrak{U}) / \mathscr{E}_{\mathscr{U}}\right)_{w c}^{\prime \prime}=\mathscr{U}_{m}^{*}(\mathfrak{U})_{c c}^{\prime \prime}=\left\{X \in \mathscr{L}^{+}\left(\mathscr{E}_{\mathfrak{U}}\right) ; \bar{X}\right.$ is affiliated with $\left.\pi(\mathfrak{U})^{\prime \prime}\right\}$.

Proof. (2) It is easily shown that $\mathscr{U}_{m}^{*}(\mathfrak{U})^{\prime \prime}=\pi(\mathfrak{U})^{\prime \prime}$ and $\pi(\mathfrak{U})^{\prime} \mathscr{E}_{\mathscr{U}}$ and $\cap \mathscr{D}(\bar{X})$ are contained in $\mathscr{F}$. Since $\mathscr{E}_{\mathscr{r}}$ is maximum in $\mathscr{F}$, $X \in \mathscr{U}_{m}^{*}(\mathfrak{R})$

it follows that $\mathscr{E}_{\mathfrak{U}}=\pi(\mathfrak{U})^{\prime} \mathscr{E}_{\mathfrak{Y}}=\bigcap_{X \in \mathscr{U}_{m}^{*}(\mathfrak{X})} \mathscr{D}(\bar{X})$, which implies that $\mathscr{U}_{m}^{*}(\mathfrak{U})$ is a closed $E W^{*}$-algebra on $\mathscr{E}_{\mathfrak{U}}$ over $\pi(\mathfrak{U})^{\prime \prime}$. It is clear that $\mathscr{U}_{m}^{*}(\mathfrak{U})$ is minimum among $E W^{*}$-algebras on $\mathscr{E}_{\mathfrak{U}}$ over $\pi(\mathfrak{U})^{\prime \prime}$ containing $\hat{\pi}(\mathfrak{U}) / \mathscr{E}_{\mathscr{C}}$ 。

(3) As stated in Section 2, it follows that $\mathscr{U}_{M}^{*}(\mathfrak{U})=\left(\mathscr{U}_{m}^{*}(\mathfrak{U})\right)_{c c}{ }_{c c}=$ $\left(\hat{\pi}(\mathfrak{U}) / \mathscr{E}_{\mathscr{V}}\right)_{w c}^{\prime \prime}=\left\{X \in \mathscr{L}^{+}\left(\mathscr{E}_{\mathscr{U}}\right) ; \quad \bar{X}\right.$ is affliated with $\left.\pi(\mathfrak{U})^{\prime \prime}\right\}$, which implies that $\mathscr{U}_{M}^{*}(\mathfrak{U})$ is maximum among $E W^{*}$-algebras on $\mathscr{E}_{\mathfrak{T}}$ over $\pi(\mathfrak{U})^{\prime \prime}$ containing $\hat{\pi}(\mathfrak{U}) / \mathscr{E}_{\mathfrak{R}}$.

(1) Since $\mathscr{U}_{m}^{*}(\mathfrak{U})$ is a closed $E W^{*}$-algebra on $\mathscr{E}_{\mathfrak{r}}$, it follows from ([9] Theorem 2.3) that

$$
\overline{\hat{\pi}\left(\xi^{*}\right)} \supset \overline{\hat{\pi}\left(\xi^{*}\right) / \mathscr{E}_{\mathfrak{r}}}=\left(\hat{\pi}(\xi) / \mathscr{E}_{\mathfrak{r}}\right) * \supset \hat{\pi}(\xi) *
$$

for each $\xi \in \mathfrak{A}$. Hence, $\hat{\pi}$ is standard.

By Lemma 6.2 we have the following

Corollary 6.3. Suppose $\mathfrak{X}$ is an unbounded left Hilbert algebra in $\mathfrak{S}$ with EW*-extendable left regular representation. Then the following statements are equivalent.

(1) $\hat{\pi}=\pi$; that is, $\pi(\mathfrak{U})^{\prime} \mathscr{D}(\pi)=\mathscr{D}(\pi)$.

(2) $\pi$ is self-adjoint.

(3) $\pi$ is standard.

Proposition 6.4. Suppose $\mathfrak{A}$ is an unbounded left Hilbert algebra in $\mathfrak{S}$ with $E W^{*}$-extendable left regular representation such that $\pi(\mathfrak{Y})^{\prime \prime}$ is semifinite. Then the following statements hold.

(1) $\mathfrak{X}$ is modular.

(2) Put

$$
\sigma_{t}(X)=\Delta^{\prime \prime i t} X \Delta^{\prime \prime}-i t
$$

for $t \in \mathbb{R}$ and $X \in \mathscr{U}(\mathfrak{U})\left(\operatorname{resp} . \mathscr{U}_{m}^{*}(\mathfrak{X}), \mathscr{U}_{M}^{*}(\mathfrak{U})\right)$. Then $\left\{\sigma_{t}\right\}_{t \in R}$ is a one-parameter group of automorphisms of the left generalized von Neumann 
algebra $\mathscr{U}(\mathfrak{U}) \quad\left(\operatorname{resp} . \mathscr{U}_{m}^{*}(\mathfrak{U}), \mathscr{U}_{M}^{*}(\mathfrak{U})\right)$.

Proof. It follows from ([32] Theorem 14.2) that $\Delta^{\prime \prime \frac{1}{2}}=K^{-1} \cdot K^{\prime}$ for some positive self-adjoint operator $K^{\prime}$ in $\mathfrak{S}$ affiliated with $\pi(\mathfrak{U})^{\prime}$ and $K=J_{\mathfrak{r}} K^{\prime} J_{\mathfrak{T}}$, which implies that $\mathfrak{U} \subset \mathscr{E}_{\mathfrak{Y}} \subset \mathscr{D}(\hat{\pi}), \quad \hat{\pi}(\mathfrak{U}) \mathscr{E}_{\mathfrak{Y}} \subset \mathscr{E}_{\mathfrak{Y}}$ and $\Delta^{\prime \prime i t} \mathscr{E}_{\mathscr{U}}=\mathscr{E}_{\mathfrak{Y}}$ for all $t \in \boldsymbol{R}$. Hence, $\mathfrak{U}$ is modular. It follows from Theorem 3.3, (2) that $\left\{\sigma_{t}\right\}$ is a one-parameter group of automorphisms of $\mathscr{U}(\mathfrak{U})$. Since $K^{2 i t} \in \pi(\mathfrak{U})^{\prime \prime}$ and $\Delta^{\prime \prime i t} \mathscr{U}_{m}^{*}(\mathfrak{U}) \Delta^{\prime \prime}-i t=$ $K^{-2 i t} \mathscr{U}_{m}^{*}(\mathfrak{U}) K^{2 i t}=\mathscr{U}_{m}^{*}(\mathfrak{U})$ for all $t \in \boldsymbol{R}$, it follows that $\left\{\sigma_{t}\right\}$ is a oneparameter group of automorphisms of $\mathscr{U}_{m}^{*}(\mathfrak{U})$. For $\mathscr{U}_{M}^{*}(\mathfrak{U})$ we have the same result as $\mathscr{U}_{m}^{*}(\mathfrak{U})$.

We give some examples of unbounded left Hilbert algebras with $E W^{*}$-extendable left regular representation.

We first consider unbounded generalization of Hilbert algebras.

Definition 6.5. A generalized (resp. unbounded) left Hilbert algebra $\mathfrak{A}$ is said to be a generalized (resp. unbounded) Hilbert algebra if $\Delta_{\mathfrak{A}}=I$. $A$ generalized Hilbert algebra $\mathfrak{U}$ in $\mathfrak{S}$ is said to be an extended Hilbert algebra if $\mathfrak{U}_{0}^{2}$ is dense in $\mathfrak{S}$, where $\mathfrak{U}_{0}=\{\xi \in \mathfrak{N} ; \overline{\pi(\xi)} \in \mathscr{B}(\mathfrak{S})\}$.

Suppose $\mathfrak{A}$ is an extended Hilbert algebra in $\mathfrak{S}$. Then $\mathfrak{U}_{0}$ is a Hilbert algebra in $\mathfrak{S}$ and $\mathfrak{A}$ is an unbounded Hilbert algebra with standard left regular representation satisfying $\mathfrak{U}_{0} \subset \mathfrak{U}^{\prime} \subset \mathfrak{U}_{0}^{\prime \prime}$. In [9] the structure of extended Hilbert algebras has been decided. Further, the following relation between extended Hilbert algebras and unbounded Hilbert algebras holds [14].

Proposition 6.6. Let $\mathfrak{A}$ be a generalized Hilbert algebra. Then the following statements are equivalent.

(1) $\mathfrak{U}$ is an unbounded Hilbert algebra.

(2) $J_{\mathfrak{r}} \pi(\mathfrak{U})^{\prime} J_{\mathfrak{r}}=\pi(\mathfrak{U})^{\prime \prime}$.

(3) $\pi(\mathfrak{U})^{\prime}$ is a von Neumann algebra, $\hat{\pi}$ is standard and $\mathfrak{X}$ is a *-subalgebra of an extended Hilbert algebra.

In this case, $\mathfrak{A}$ is an unbounded left Hilbert algebra with $E W^{*}$ extendable left regular representation.

We next consider unbounded left Hilbert algebras constructed by 
unbounded Hilbert algebras and positive self-adjoint operators.

Let $\left(\mathscr{B}, K^{\prime}\right)$ be given, where $\mathscr{B}$ is an unbounded Hilbert algebra in a Hilbert space $\mathfrak{E}$ and $K^{\prime}$ is a non-singular positive self-adjoint operator in $\mathscr{S}$ affiliated with $\pi_{\mathscr{B}}(\mathscr{B})^{\prime}$ satisfying $\mathscr{B} \subset \mathscr{D}\left(K^{\prime}\right)$. We now define a multiplication and an involution on $K^{\prime} \mathscr{B}$ by

$$
\begin{aligned}
& \left(K^{\prime} \xi\right)\left(K^{\prime} \eta\right)=K^{\prime} \xi \eta, \\
& \left(K^{\prime} \xi\right)^{\sharp}=K^{\prime} \xi^{*}
\end{aligned}
$$

for $\xi, \eta \in \mathscr{B}$. Then $K^{\prime} \mathscr{B}$ is a $*$-algebra satisfying

$$
K^{\prime} \mathscr{B} \subset \mathscr{D}\left(\hat{\pi}_{\mathscr{B}}\right) \text { and } \hat{\pi}_{\mathscr{B}}(\xi) K^{\prime} \eta=K^{\prime} \xi \eta
$$

for each $\xi, \eta \in \mathscr{B}$. Let $K^{\prime}=\int_{0}^{\infty} \lambda d E^{\prime}(\lambda)$ and $K \equiv J_{\mathscr{B}} K^{\prime} J_{\mathscr{B}}=\int_{0}^{\infty} \lambda d E(\lambda)$ be the spectral resolutions of $K^{\prime}$ and $K$, respectively and put $E^{\prime}(n)$ $=\int_{0}^{n} d E^{\prime}(\lambda)$ and $E(n)=\int_{0}^{n} d E(\lambda)$ for $n \in \mathbb{N}$. We consider when $K^{\prime} \mathscr{B}$ is an unbounded left Hilbert algebra with $E W^{*}$-extendable left regular representation.

Theorem 6.7. Suppose $\left(\mathscr{B}, K^{\prime}\right)$ is a couple of an unbounded Hilbert algebra $\mathscr{B}$ in a Hilbert space $\mathfrak{S}_{\mathrm{E}}$ with standard left regular representation and a non-singular positive self-adjoint operator $K^{\prime}$ in $\mathfrak{S}$ affiliated with $\pi_{\mathscr{B}}(\mathscr{B})^{\prime}$ such that $\mathscr{B} \subset \mathscr{D}\left(K^{\prime}\right)$ and $\bar{K}^{\prime}{ }_{\mathscr{B}}{ }^{t} \mathscr{\mathscr { B }}=\mathscr{D}\left(\pi_{\mathscr{B}}\right)$. Then $K^{\prime} \mathscr{B}$ is an unbounded left Hilbert algebra in $\mathfrak{S}$ satisfying $\pi_{K^{\prime} \mathscr{G}}\left(K^{\prime} \tilde{\xi}\right)=\pi_{\mathscr{B}}(\xi)$ for each $\xi \in \mathscr{B}$ and $S_{K^{\prime} \mathscr{B}}^{\prime \prime}=J_{\mathscr{B}} K \cdot K^{\prime-1}$. Further, suppose $\mathscr{B} " \subset \mathscr{D}\left(K^{\prime}\right) ;$ for example, $\mathscr{B}$ has identity. Then $K^{\prime} \mathscr{B}$ is an unbounded left Hilbert algebra in $\mathfrak{S}$ with an $E W^{*}$-extendable left regular representation.

Proof. Since $K^{\prime} \mathscr{B}$ is dense in $\left(\mathscr{D}\left(\pi_{\mathscr{B}}\right), t_{\pi_{\mathscr{B}}}\right)$ and the statement (6. 1), it follows that $K^{\prime} \mathscr{B}$ is a $*$-algebra in $\mathscr{C}$ satisfying $\pi_{K^{\prime} \mathscr{B}}\left(K^{\prime} \xi\right)$ $=\pi_{\mathscr{R}}(\xi)$ for every $\xi \in \mathscr{B},\left(K^{\prime} \mathscr{B}\right)^{2}$ is dense in $\mathfrak{g}$ and $\left(\left(K^{\prime} \xi\right)\left(K^{\prime} \eta\right) \mid K^{\prime} \zeta\right)$ $=\left(K^{\prime} \eta \mid\left(K^{\prime} \xi\right)^{\sharp}\left(K^{\prime} \zeta\right)\right)$ for each $\xi, \eta, \zeta \in \mathscr{B}$. Since

$$
\cup_{n, m \in N} E(n) E^{\prime}(m) \mathscr{B}^{\prime \prime} \subset \mathscr{B}^{\prime \prime} \cap \mathscr{D}(K) \cap \mathscr{D}\left(K^{\prime}\right),
$$

it follows that $\mathscr{B}^{\prime \prime} \cap \mathscr{D}(K) \cap \mathscr{D}\left(K^{\prime}\right)$ is a Hilbert algebra in $\mathfrak{S}$. Further, since $\mathscr{B}^{\prime \prime} \subset \mathscr{D}\left(\pi_{\mathscr{B}}\right)$ [14] and (6.1) we have

$$
\begin{aligned}
\left(\pi_{\mathscr{F}}(\xi) \eta \mid K x\right) & =\left(J K x \mid \eta^{*} \xi^{*}\right) \\
& =\left(x^{*} \mid K^{\prime} \eta^{*} \xi^{*}\right) \\
& =\left(x^{*} \mid \pi_{\mathscr{F}}\left(\eta^{*}\right) K^{\prime} \xi^{*}\right)
\end{aligned}
$$




$$
\begin{aligned}
& =\left(\pi_{\mathscr{B}}(\eta) x^{*} \mid K^{\prime} \xi^{*}\right) \\
& =\left(\eta \mid \rho_{\mathscr{B}}(x) K^{\prime} \xi^{*}\right)
\end{aligned}
$$

for each $\xi, \eta \in \mathscr{B}$ and $x \in \mathscr{B}^{\prime \prime} \cap \mathscr{D}(K) \cap \mathscr{D}\left(K^{\prime}\right)$, where $\rho_{\mathscr{\mathscr { B }}}^{\prime \prime}$ is the right regular representation of $\mathscr{B}^{\prime \prime}$, and hence $K\left(\mathscr{B}^{\prime \prime} \cap \mathscr{D}(K) \cap \mathscr{D}\left(K^{\prime}\right)\right) \subset$ $\mathscr{D}\left(\pi_{\mathscr{B}}\right)$ and $\pi_{\mathscr{B}}(\xi) K x=\rho_{\mathscr{B}}(x) K^{\prime} \xi$ for each $\xi \in \mathscr{B}$ and $x \in \mathscr{B}^{\prime \prime} \cap \mathscr{D}(K)$ $\cap \mathscr{D}\left(K^{\prime}\right)$, which implies that

$$
\left(K x \mid K^{\prime} \xi \eta\right)=\left(K^{\prime} \eta^{*} \xi^{*} \mid K x^{*}\right)
$$

and

$$
\pi_{K^{\prime} \mathscr{B}}^{\prime}(K x) K^{\prime} \xi=\pi_{\mathscr{B}}(\xi) K x=\rho_{\mathscr{B}}(x) K^{\prime} \xi
$$

for each $\xi, \eta \in \mathscr{B}$ and $x \in \mathscr{B}^{\prime \prime} \cap \mathscr{D}(K) \cap \mathscr{D}\left(K^{\prime}\right)$. Hence, we have

$$
\begin{aligned}
& K\left(\mathscr{B}^{\prime \prime} \cap \mathscr{D}(K) \cap \mathscr{D}\left(K^{\prime}\right)\right) \subset\left(K^{\prime} \mathscr{B}\right)^{\prime}, \\
& (K x)^{b}=K x^{*} \quad \text { and } \quad \pi_{K^{\prime} \mathscr{B}}^{\prime}(K x)=\rho_{\mathscr{B}^{\prime \prime}}(x)
\end{aligned}
$$

for every $x \in \mathscr{B}^{\prime \prime} \cap \mathscr{D}(K) \cap \mathscr{D}\left(K^{\prime}\right)$. It is clear that $\left(\underset{n, m}{\cup} E(n) E^{\prime}(m) \mathscr{B}^{\prime \prime}\right)^{2}$ is dense in the Hilbert space $\mathscr{D}(K)$, and hence by (6.2) $K\left(\mathscr{B}^{\prime \prime} \cap\right.$ $\left.\mathscr{D}(K) \cap \mathscr{D}\left(K^{\prime}\right)\right)^{2}$ is dense in $\mathscr{S}$. It hence follows from (6.3) that $\left(K^{\prime} \mathscr{B}\right)^{\prime 2}$ is dense in $\mathfrak{C}$, which implies that $K^{\prime} \mathscr{B}$ is an unbounded left Hilbert algebra in $\mathfrak{S}$. We next show $S_{K^{\prime} \mathscr{B}}^{\prime \prime}=J_{\mathscr{B}} K \cdot K^{\prime-1}$. Take arbitrary $x \in\left(K^{\prime} \mathscr{B}\right)^{\prime \prime}$ and $y \in \mathscr{D}\left(K K^{\prime-1}\right)$. Then, $y=K^{\prime} z$ for some $z \in \mathscr{D}(K)$ $\cap \mathscr{D}\left(K^{\prime}\right)$. It follows from (6.2) that $\mathscr{B}^{\prime \prime} \cap \mathscr{D}(K) \cap \mathscr{D}\left(K^{\prime}\right)$ is dense in the normed space $\left(\mathscr{D}(K) \cap \mathscr{D}\left(K^{\prime}\right),\|\| \|||\right)$ where $\|x\| \mid=\|K x\|+\left\|K^{\prime} x\right\|$ for $x \in \mathscr{D}(K) \cap \mathscr{D}\left(K^{\prime}\right)$, so that there exists a sequence $\left\{\xi_{n}\right\}$ in $\mathscr{B}^{\prime \prime} \cap$ $\mathscr{D}(K) \cap \mathscr{D}\left(K^{\prime}\right)$ such that $\lim _{n \rightarrow \infty} K \xi_{n}=K z$ and $\lim _{n \rightarrow \infty} K^{\prime} \xi_{n}=K^{\prime} z$. Then we have

$$
\begin{aligned}
\left(K K^{\prime-1} y \mid x\right) & =\lim _{n \rightarrow \infty}\left(K \xi_{n} \mid x\right) \\
& =\lim _{n \rightarrow \infty}\left(S_{K^{\prime} \mathscr{B}}^{\prime \prime} x \mid K \xi_{n}^{*}\right) \\
& =\left(S_{K^{\prime} \mathscr{B}}^{\prime \prime} x \mid J_{\mathscr{B}} K^{\prime} z\right) \\
& =\left(y \mid J_{\mathscr{B}} S_{K^{\prime} \mathscr{B}}^{\prime \prime} x\right),
\end{aligned}
$$

which implies that $x \in \mathscr{D}\left(\left(K K^{\prime-1}\right)^{*}\right)=\mathscr{D}\left(K \cdot K^{\prime-1}\right)$ and $K \cdot K^{\prime-1} x=J_{\mathscr{B}} S_{K^{\prime} \mathscr{B}}^{\prime \prime} x$ for every $x \in\left(K^{\prime} \mathscr{B}\right)^{\prime \prime}$, which means $S_{K^{\prime} \mathscr{B}}^{\prime \prime} \subset J_{\mathscr{B}} K \cdot K^{\prime-1}$. On the other hand, since $\mathscr{B}^{\prime \prime} \cap \mathscr{D}(K) \cap \mathscr{D}\left(K^{\prime}\right) \subset\left(K^{\prime} \mathscr{B}\right)^{\prime \prime}, \lim _{n \rightarrow \infty} K^{\prime} \xi_{n}=y$ and

$$
\lim _{n \rightarrow \infty} S_{K^{\prime} \mathscr{B}}^{\prime \prime} K^{\prime} \xi_{n}=J_{\mathscr{B}} K z,
$$

we have $J_{\mathscr{B}} K \cdot K^{\prime-1} \subset S_{K^{\prime} \mathscr{B}}^{\prime \prime}$. 
Suppose $\mathscr{B}^{\prime \prime} \subset \mathscr{D}\left(K^{\prime}\right)$. We denote by $E(\mathscr{B})$ the $*$-algebra generated by $\mathscr{B}$ and $\mathscr{B}^{\prime \prime}$. Then it follows from ([14] Theorem 3.3) and (6.1) that $E(\mathscr{B}) \subset \mathscr{D}\left(K^{\prime}\right), \quad K^{\prime} \mathscr{B} \subset K^{\prime} E(\mathscr{B}) \subset \mathscr{D}\left(\pi_{\mathscr{B}}\right), \quad \pi_{K^{\prime} \mathscr{B}}\left(K^{\prime} \mathscr{B}\right) K^{\prime} E(\mathscr{B}) \subset$ $K^{\prime} E(\mathscr{B})$ and $\pi_{K^{\prime} \mathscr{B}}\left(K^{\prime} \mathscr{B}\right)^{\prime \prime} K^{\prime} E(\mathscr{B}) \subset K^{\prime} E(\mathscr{B})$, which implies that $K^{\prime} \mathscr{B}$ is an unbounded left Hilbert algebra with $E W^{*}$-extendable left regular representation. This completes the proof.

By Proposition 6.4 and Theorem 6.7 we have the following

Corollary 6.8. Suppose $\left(\mathscr{B}, K^{\prime}\right)$ is of Theorem 6.7 and $\mathscr{B} " \subset \mathscr{D}\left(K^{\prime}\right)$. Then $K^{\prime} \mathscr{B}$ is a modular unbounded left Hilbert algebra with standard left regular representation. Furiher, suppose $\mathscr{B}$ is dense in the normed space $\left(\mathscr{D}(K) \cap \mathscr{D}\left(K^{\prime}\right),||||||\right)$. Then $K^{\prime} \mathscr{B}$ is a standard unbounded left Hilbert algebra.

We next consider the converse of Theorem 6.7: When is an unbounded left Hilbert algebra $\mathfrak{2}$ represented as $\mathfrak{U}=K^{\prime} \mathscr{B}$ ?

Theorem 6.9. Suppose $\mathfrak{X}$ is an unbounded left Hilbert algebra in a Hilbert space $\mathfrak{S}$ satisfying $\pi_{\mathfrak{r}}(\mathfrak{U})^{\prime \prime}$ is a semifinite von Neumann algebra, $\overline{\pi_{\mathfrak{Y}}(\mathfrak{X})} \subset L^{2}\left(\tau^{\prime \prime}\right)$ and $\overline{\pi_{\mathfrak{Y}}\left(\mathfrak{X}^{2}\right)}$ is dense in $L^{2}\left(\tau^{\prime \prime}\right)$, where $\tau^{\prime \prime}$ is a faithful normal semifinite trace on $\pi_{\mathfrak{U}}(\mathfrak{U})^{\prime \prime}$. Then $\mathfrak{U}$ is unitarily equivalent to the unbounded left Hilbert algebra $K^{\prime} \mathscr{B}$, where $\left(\mathscr{B}, K^{\prime}\right)$ is a couple of an unbounded Hilbert algebra $\mathscr{B}$ in a Hilbert space $\mathscr{K}$ with standard left regular representation and a non-singular positive self-adjoint operator $K^{\prime}$ in $\mathscr{K}$ affiliated with $\pi_{\mathscr{B}}(\mathscr{B})^{\prime}$ saitisfying $\mathscr{B} \subset \mathscr{D}\left(K^{\prime}\right)$ and $K^{\prime} \mathscr{B}$ is dense in $\left(\mathscr{D}\left(\pi_{\mathscr{B}}\right), t_{\pi_{\mathscr{B}}}\right)$.

Proof. Since $\mathfrak{A}$ is an unbounded left Hilbert algebra in $\mathfrak{g}$, it follows that $\mathfrak{U}^{\prime \prime}$ is an achieved left Hilbert algebra in $\mathfrak{E}$ such that $\pi_{\mathfrak{X}}(\mathfrak{U})^{\prime \prime}$ equals the left von Neumann algebra $\mathscr{U}_{0}\left(\mathfrak{X}^{\prime \prime}\right)$ of $\mathfrak{U}^{\prime \prime}$. We denote by $\mathscr{L}$ the set of all left bounded elements $\xi$ of $\mathfrak{S}$ with $\pi^{\prime \prime}(\xi) \in \mathfrak{N}_{\tau^{\prime \prime}}$, where $\pi^{\prime \prime}(\xi) \eta=\pi_{\mathfrak{X}}^{\prime}(\eta) \xi$ for $\eta \in \mathfrak{U}^{\prime}$ and $\mathfrak{N}_{\tau^{\prime \prime}}=\left\{X \in \pi_{\mathfrak{N}}(\mathfrak{U})^{\prime \prime}\right.$; $\left.\tau^{\prime \prime}\left(X^{*} X\right)<\infty\right\}$. Takesaki has proved in [32] that

(6. 4) $\pi^{\prime \prime}$ is a closable operator of the dense subspace $\mathscr{L}$ in $\mathfrak{2}$ onto the dense subspace $\pi^{\prime \prime}(\mathscr{L})$ in $L^{2}\left(\tau^{\prime \prime}\right)$ whose closure $\Pi$ is non-singular;

(6.5) let $\Pi=V T^{\prime}$ be the polar decomposition of $\Pi$. Then $V$ is a 
unitary operator of $\mathfrak{S}$ onto $L^{2}\left(\tau^{\prime \prime}\right)$ and $T^{\prime}$ is a non-singular positive selfadjoint operator in $\mathfrak{S}$ affliated with $\pi_{\mathfrak{O}}(\mathfrak{U})^{\prime \prime}$ satisfying $\Delta^{\prime \prime \frac{1}{2}}=T^{-1} \cdot T^{\prime}$, where $T=J_{\mathfrak{\imath}}^{\prime \prime} T^{\prime} J_{\mathfrak{U}}^{\prime \prime}$;

(6.6) let $\rho_{0}$ be the representation of $\pi_{\mathfrak{r}}(\mathfrak{U})^{\prime \prime}$ on $L^{2}\left(\tau^{\prime \prime}\right)$ defined by: $\rho_{0}(X) Y=X Y$ for $X \in \pi_{\mathfrak{r}}(\mathfrak{Y})^{\prime \prime}$ and $Y \in \mathfrak{N}_{\tau^{\prime \prime}}$. Then, the unitary operator $V$ induces a spatial isomorphism between $\pi_{\mathfrak{U}}(\mathfrak{U})^{\prime \prime}$ and $\rho_{0}\left(\pi_{\mathfrak{U}}(\mathfrak{U})^{\prime \prime}\right)$ such that $V \pi^{\prime \prime}(\xi) V^{*}=\rho_{0}\left(\pi^{\prime \prime}(\xi)\right)$ for $\xi \in \mathscr{L}$;

(6. 7) $\mathfrak{U}^{\prime \prime} \cap \mathscr{L}$ is dense in the Hilbert space $\mathscr{D}\left(T^{\prime}\right)$.

Let $\Lambda$ be the inverse of $\Pi$ and $\Lambda=U K^{\prime}$ be the polar decomposition of $\Lambda$. Then we have $U=V^{*}$ and $K^{\prime}=U^{*} T^{-1} U$. It follows from (6.5) that $U$ is a unitary operator of $L^{2}\left(\tau^{\prime \prime}\right)$ onto $\mathfrak{S}$ and $K^{\prime}$ is a nonsingular positive self-adjoint operator in $L^{2}\left(\tau^{\prime \prime}\right)$ affiliated with the von Neumann algebra $\rho_{0}\left(\pi_{\mathfrak{I}}(\mathfrak{U})^{\prime \prime}\right)$. Since $\overline{\pi_{\mathfrak{H}}(\mathfrak{U})} \subset L^{2}\left(\tau^{\prime \prime}\right)$, it follows from ([9] Theorem 5) that $\pi_{\mathfrak{R}}$ is standard, which implies that $\overline{\pi_{\mathfrak{U}}(\xi)}+\overline{\pi_{\mathfrak{U}}(\eta)} \equiv \overline{\overline{\pi_{\mathfrak{U}}(\xi)}+\overline{\pi_{\mathfrak{U}}(\eta)}}=\overline{\pi_{\mathfrak{U}}(\xi+\eta)}, \quad \lambda \cdot \overline{\pi_{\mathfrak{U}}(\xi)} \equiv \overline{\overline{\pi_{\mathfrak{U}}(\xi)}}=\overline{\pi_{\mathscr{U}}(\lambda \xi)}$, $\overline{\pi_{\mathscr{U}}(\xi)} \cdot \overline{\pi_{\mathfrak{U}}(\eta)} \equiv \overline{\overline{\pi_{\mathfrak{U}}(\xi)} \overline{\pi_{\mathfrak{U}}(\eta)}}=\overline{\pi_{\mathfrak{U}}(\xi \eta)}$ and $\pi_{\mathfrak{U}}(\xi) *=\overline{\pi_{\mathfrak{U}}\left(\xi^{\sharp}\right)}$ for each $\xi, \eta \in \mathfrak{U}$. Hence, $\mathscr{B} \equiv \overline{\pi_{\mathfrak{I}}(\mathfrak{U})}$ is a generalized Hilbert algebra in $L^{2}\left(\tau^{\prime \prime}\right)$ equipped with the strong sum, strong scalar multiplication, strong product and adjoint. We first show

$$
\mathfrak{U} \subset \mathscr{D}(\Pi) \quad \text { and } \quad \Pi \xi=\overline{\pi_{\mathscr{U}}(\xi)}
$$

for every $\xi \in \mathfrak{X}$. Take arbitrary $\xi \in \mathfrak{X}$. Let $\overline{\pi_{\mathfrak{N}}(\xi)}=U_{\xi}\left|\overline{\pi_{\mathfrak{X}}(\xi)}\right|$ be the polar decomposition of $\overline{\pi_{\mathfrak{U}}(\xi)}$ and $\left|\overline{\pi_{\mathfrak{U}}(\xi)}\right|=\int_{0}^{\infty} \lambda d E_{\xi}(\lambda)$ be the spectral resolution of $\left|\overline{\pi_{\mathfrak{r}}(\xi)}\right|$. Then it follows that $E_{\xi^{*}}(n) \xi \in \mathscr{L}$ and $\pi^{\prime \prime}\left(E_{\xi^{*}}(n) \xi\right)=E_{\xi^{*}}(n) \overline{\pi_{\mathscr{N}}(\xi)}=E_{\xi^{*}}(n)\left|\overline{\pi_{\mathfrak{R}}\left(\xi^{*}\right)}\right| U_{\xi^{*}}^{*}$ for $n \in N$, so that

$$
\begin{aligned}
& \lim _{n \rightarrow \infty} E_{\xi^{*}}(n) \xi=\xi, \\
& \begin{aligned}
\lim _{n \rightarrow \infty}\left\|\Pi E_{\xi^{*}}(n) \xi-\overline{\pi_{\mathfrak{X}}(\xi)}\right\|_{2}^{2} & =\lim _{n \rightarrow \infty}\left\|\pi^{\prime \prime}\left(E_{\xi^{*}}(n) \xi\right)-\overline{\pi_{\mathfrak{U}}(\xi)}\right\|_{2}^{2} \\
& =\lim _{n \rightarrow \infty} \mu_{\tau^{\prime \prime}}\left(\left.\left(I-E_{\xi^{*}}(n)\right) \overline{\mid \pi_{\mathfrak{U}}(\xi)}\right|^{2}\right) \\
& =0,
\end{aligned}
\end{aligned}
$$

where \|\|$_{2}$ is the $L^{2}$-norm on $L^{2}\left(\tau^{\prime \prime}\right)$ and $\mu_{\tau^{\prime \prime}}$ is the integral on $L^{1}\left(\tau^{\prime \prime}\right)$ [26]. Hence, $\xi \in \mathscr{D}(\Pi)$ and $\Pi \xi=\overline{\pi_{\mathscr{U}}(\xi)}$.

Since $\rho_{0}\left(\pi_{\mathfrak{U}}(\mathfrak{U})^{\prime \prime}\right)^{\prime}=\rho_{0}^{\prime}\left(\pi_{\mathfrak{V}}(\mathfrak{U})^{\prime \prime}\right)$, where $\rho_{0}^{\prime}$ is the antirepresentation of $\pi_{\mathfrak{U}}(\mathfrak{U})^{\prime \prime}$ on $L^{2}\left(\tau^{\prime \prime}\right)$ defined by: $\rho_{0}^{\prime}(X) Y=Y X$ for $X \in \pi_{\mathfrak{V}}(\mathfrak{H})^{\prime \prime}$ and $Y \in \mathfrak{A}_{\tau^{\prime \prime}}$, it is easily shown that $\rho_{0}\left(\pi_{\mathscr{U}}(\mathfrak{U})^{\prime \prime}\right)^{\prime} \subset \pi_{\mathscr{B}}(\mathscr{B})^{\prime}$, so that $K^{\prime}$ is 
affiliated with $\pi_{\mathscr{B}}(\mathscr{B})^{\prime}$. It hence follows from (6.8) that $\mathscr{B} \subset \mathscr{D}\left(K^{\prime}\right)$ and $K^{\prime} \mathscr{B}$ is a generalized left Hilbert algebra in $L^{2}(\tau)$, equipped

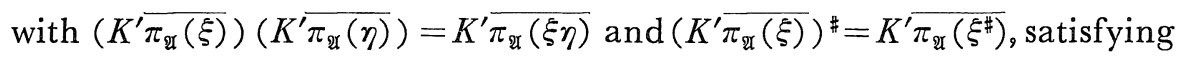

$$
\pi_{K^{\prime} \mathscr{B}}\left(K^{\prime} \overline{\pi_{\mathscr{A}}(\xi)}\right) \subset \pi_{\mathscr{B}}\left(\overline{\pi_{\mathscr{P}}(\xi)}\right)
$$

for every $\xi \in \mathfrak{A}$. Further, since $U \pi_{K^{\prime} \mathscr{B}}\left(K^{\prime} \overline{\pi_{\mathfrak{I}}(\xi)}\right)\left(K^{\prime} \overline{\pi_{\mathfrak{U}}(\eta)}\right)=U K^{\prime} \overline{\pi_{\mathfrak{U}}(\xi \eta)}=$ $\pi_{\mathscr{U}}(\xi) \eta$ for every $\xi, \eta \in \mathfrak{N}$, it follows that $U \pi_{K^{\prime} \mathscr{B}}\left(K^{\prime} \overline{\pi_{\mathfrak{Y}}(\xi)}\right) U^{*}=\pi_{\mathscr{Y}}(\xi)$ for every $\xi \in \mathfrak{U}$, so that $\pi_{K^{\prime} \mathscr{O}}$ is standard by the standardness of $\pi_{\mathscr{N}}$, which implies by (6.9) that

$$
\pi_{K^{\prime} \mathscr{B}}\left(K^{\prime} \overline{\pi_{\mathscr{P}}(\xi)}\right)=\pi_{\mathscr{B}}\left(\overline{\pi_{\mathscr{P}}(\xi)}\right)
$$

for every $\xi \in \mathscr{B}$. Hence, $\pi_{\mathscr{B}}$ is standard, which implies that $K^{\prime} \mathscr{B}$ is dense in $\left(\mathscr{D}\left(\pi_{\mathscr{B}}\right), t_{\pi_{\mathscr{B}}}\right)$.

Since $\pi_{\mathfrak{O}}$ is unitarily equivalent to $\pi_{K^{\prime} \mathscr{B}}$ and the statements (6.6), (6.10), we have $\pi_{\mathscr{B}}(\mathscr{B})^{\prime \prime}=\rho_{0}\left(\pi_{\mathscr{U}}(\mathfrak{H})^{\prime \prime}\right)$. We now show that $\mathscr{B}$ is an unbounded Hilbert algebra in $L^{2}\left(\tau^{\prime \prime}\right)$. Take arbitrary $X \in \mathfrak{N}_{\tau^{\prime \prime}}$. Then it follows that $X \in \mathscr{D}\left(\pi_{\mathscr{B}}\right)$ and $\pi_{\mathscr{B}}\left(\overline{\pi_{\mathfrak{U}}(\xi)}\right) X=\overline{\pi_{\mathfrak{X}}(\xi)} X=\rho_{0}^{\prime}(X) \overline{\pi_{\mathfrak{U}}(\xi)}$ for every $\xi \in \mathfrak{N}$, so that $\pi_{\mathscr{B}}^{\prime}(X)=\rho_{0}^{\prime}(X)$. Hence, $\mathfrak{N}_{\tau^{\prime \prime}} \subset \mathscr{B}^{\prime}$. Since $\mathfrak{N}_{\tau^{\prime \prime}}$ is an achieved Hilbert algebra in $L^{2}\left(\tau^{\prime \prime}\right)$, it follows that $\mathscr{B}$ is an unbounded

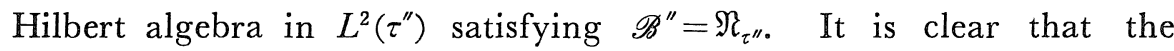
unitary operator $U$ of $L^{2}\left(\tau^{\prime \prime}\right)$ onto $\mathfrak{S}$ induces an isomorphism between the unbounded left Hilbert algebras $K^{\prime} \mathscr{B}$ and $\mathfrak{A}$. This completes the proof.

Let $\mathscr{A}$ be a $*$-algebra with identity $e$ and $\tau$ be a tracial positive linear functional on $\mathscr{A}$ (that is, $\tau\left(x^{*} x\right)=\tau\left(x x^{*}\right)$ for each $\left.x \in \mathscr{A}\right)$. Then, the pre-Hilbert space $\lambda_{\tau}(\mathscr{A})$ in the Hilbert space $\mathfrak{S}_{\tau}$ is a generalized Hilbert algebra in $\mathfrak{Q}_{\tau}$ equipped with the multiplication $\lambda_{\tau}(x) \lambda_{\tau}(y)=\lambda_{\iota}(x y)$ and the involution $\lambda_{\tau}(x)^{*}=\lambda_{\tau}\left(x^{*}\right)$. By ([14] Theorem 3.3) $\lambda_{\tau}(\mathscr{A})$ is an unbounded Hilbert algebra in $\mathfrak{S}_{\tau}$ if and only if $\tau$ is standard if and only if $J_{\tau} \pi_{\tau}(\mathscr{A})^{\prime} J_{\tau}=\pi_{\tau}(\mathscr{A})^{\prime \prime}$.

Suppose $\tau$ is a standard tracial positive linear functional on $\mathscr{A}$ and $K^{\prime}$ is a positive self-adjoint operator in $\mathfrak{L}_{\tau}$ affiliated with $\pi_{\tau}(\mathscr{A})^{\prime}$ satisfying $\mathscr{D}\left(K^{\prime}\right) \supset \lambda_{\tau}(\mathscr{A})$. Then a positive linear functional $K^{\prime} \tau K^{\prime}$ on $\mathscr{A}$ is defined by

$$
\left(K^{\prime} \tau K^{\prime}\right)(x)=\left(K^{\prime} \lambda_{\tau}(x) \mid K^{\prime} \lambda_{\tau}(e)\right)
$$

for $x \in \mathscr{A}$. By Corollary 6.8 and Theorem 6.9 we have the following 
Corollary 6.10. Let $\phi$ be a positive linear functional on a *-algebra $\mathscr{A}$ with identity $e$. Then the following statements are equivalent.

(1) $\phi=K^{\prime} \tau K^{\prime}$, where $\tau$ is a standard tracial positive linear functional on $\mathscr{A}$ and $K^{\prime}$ is a non-singular positive self-adjoint operator in $\mathfrak{S}_{\tau}$ affiliated with $\pi_{\tau}(\mathscr{A})^{\prime}$ satisfying $\lambda_{\tau}(\mathscr{A}) \subset \mathscr{D}\left(K^{\prime}\right)$ and $K^{\prime} \lambda_{\tau}(\mathscr{A})$ is dense in $\left(\mathscr{D}\left(\pi_{\tau}\right), t_{\pi_{\tau}}\right)$.

(2) $\pi_{\phi}(\mathscr{A}) \lambda_{\phi}(e)$ is dense in $\mathfrak{K}_{\phi}$ and there exists a faithful normal finite trace $\tau^{\prime \prime}$ on $\pi_{\phi}(\mathscr{A})^{\prime \prime}$ such that $\overline{\pi_{\phi}(\mathscr{A})}$ is densely contained in the Hilbert space $L^{2}\left(\tau^{\prime \prime}\right)$.

In this case, $\phi$ is modular, and $\phi$ is standard if and only if $\lambda_{\tau}(\mathscr{A})$ is dense in the normed space $\left(\mathscr{D}(K) \cap \mathscr{D}\left(K^{\prime}\right),\||\||)\right.$, where $K=J_{\tau} K^{\prime} J_{\tau}$.

\section{References}

[1] H. Araki, Some properties of modular conjugation operator of von Neumann algebras and a non-commutative Radon-Nikodym theorem with a chain rule, Pacific J. Math., 50 (1974), 309-354.

[2] H. J. Borchers, Algebraic aspects of Wightman field theory, In Statistical Mechanics and Field Theory Lectures, 1971; Haifa Summer School, New York-Jerusalem-London, (1972), 31-79.

[3] J. Diximier, Les algebres d'opérateurs dans l'espace Hilbertian, Gausthier-Villars, Paris, 2é edition, 1969.

[4] N. Dunford and J. T. Schwartz, Linear operators, Vol.II, Interscience, New York, 1957.

[5] G. Epifanio and C. Trapani, $V^{*}$-algebras: an extension of the concept of von Neumann algebras to unbounded operators, J. Math. Phys., 25 (1984), 2633-2637.

[6] I. M. Gelfand and N. Ya. Vilenkin, Generalized functions, Vol. 4, Academic Press, New York, 1964.

[7] S. P. Gudder and W. Scruggs, Unbounded representations of *-algebras, Pacific J. Math., 70 (1977), 369-382.

[8] S. P. Gudder and R. L. Hudson, A noncommutative probability theory, Trans. Amer. Math. Soc., 245 (1978), 1-41.

[9] A. Inoue, On a class of unbounded operator algebras I, II, III, Pacific J. Math., 65 (1976), 77-95 ; 66 (1976), 411-431; 69 (1977), 105-115.

[10] - A commutant of an unbounded operator algebra, Proc. Amer. Math. Soc., 69 (1978), 97-102.

[11] - Unbounded generalization of left Hilbert algebras I, II, J. Functional Analysis, 34 (1979), 339-362: J. Functional Analysis, 35 (1980), 230-250.

[12] - Standard representations of commutative *-algebras, Fukuoka Univ. Sci. Rep., 14. (1984), 61-66.

[13] — Self-adjointness of *-representations generated by positive linear functionals, Proc. Amer. Math. Soc., 93 (1985), 643-647.

[14] - An unbounded generalization of Hilbert algebras, Preprint.

[15] — and K. Takesue, Self-adjoint representations of polynomial algebras, Trans. Amer. Math. Soc., 280 (1983), 393-400.

[16] A. Inoue, H. Ueda and T. Yamauchi, Commutants and bicommutants of algebras of unbounded operators, to appear in J. Moth. Phys.

[17] K. Katavolos and I. Koch, Extension of Tomita-Takesaki theory to the unbounded 
algebra of the canonical commutation relations, Rep. Math. Phys., 16 (1979), 335-352.

[18] G. Lassner, Topological algebras of operators, Rep. Math. Phys., 3 (1972), 279-293.

[19] - Topological algebras and their applications in quantum statistics, Wiss. $Z$. Karl-Marx-Univ. Leipzig, Math.-Naturwiss. R., 30 (1981), 572-595.

[20] G. Lassner and W. Timmermann, Normal states on algebras of unbounded operators, Rep. Math. Phys., 3 (1972), 295-305.

[21] F. Mathot, Topological properties of unbounded bicommutants, J. Math. Phys., 26 (1985), 1118-1124.

[22] A. E. Nussbaum, On the integral representation of positive linear functionals, Trans. Amer. Math. Soc., 128 (1967), 460-473.

[23] R. T. Powers, Self-adjoint algebras of unbounded operators I, II, Commun. Math. Phys., 21 (1971), 85-124: Trans. Amer. Math. Soc., 187 (1974), 261-293.

[24] , Algebras of unbounded operators, Proc. Sym. Pure Math., 38 (1982), 389406.

[25] M. A. Rieffel and A. Van Daele, A bounded operator approach to Tomita-Takesaki theory, Pacific J. Math., 69 (1977), 187-221.

[26] I. E. Segal, A non-commutative extention of abstract integration, Ann. Math., 57 (1953), 401-457.

[27] K. Schmüdgen, The order structure of topological*-algebras of unbounded operators, Rep. Math. Phys., 7 (1975), 215-227.

[28] - On trace representation of linear functionals on unbounded operator algebras, Commun. Math. Phys., 63 (1978), 113-130.

[29] M. Schröder and W. Timmermann, Invariance of domains and automorphisms in algebras of unbounded operators, In: Proc. Int. Conf. on Operator algebras and Group Representations, Romania, (1980), 134-139.

[30] T. Sherman, Positive linear functionals on *-algebras of unbounded operators, $J$. Math. Anal. Appl., 22 (1968), 285-318.

[31] S. Stratila and L. Zsidó, Lectures on von Neumann algebras, Editura Academiei \& Abacus Press, 1979.

[32] M. Takesaki, Tomita's theory of modular Hilbert algebras and its applications, Lecture Notes in Mathematics, 128 Springer, 1970.

[33] K. Takesue, Standard representations induced by positive linear functionals, Mem. Fac. Sci. Kyushu Univ., 37 (1983), 211-225.

[34] M. Tomita, Standard froms of von Neumann algebras, The Vth functional analysis symposium of the Math. Soc. of Japan, Sendai, 1967.

[35] A. Van Daele, A new approach to the Tomita-Takesaki Theory of generalized Hilbert algebras, J. Functional Analysis, 15 (1974), 378-393.

[36] S. L. Woronwicz, The quantum moment problem I, II, Rep. Math. Phys., $\mathbb{1}$ (1970), 135-145; Rep. Math. Phys., $\mathbb{1}$ (1971), 175-183. 
